\title{
Universal Scaling of \\ Linear and Nonlinear Rheological Properties of Semidilute and Concentrated Polymer Solutions
}

\author{
by \\ Youngsuk Heo
}

A dissertation submitted in partial fulfillment

of the requirements for the degree of

Doctor of Philosophy

(Macromolecular Science and Engineering)

in The University of Michigan

2008

Doctoral Committee:

Professor Ronald G. Larson, Chair

Professor Peter F. Green

Professor David C. Martin

Associate Professor Michael J. Solomon 


\section{(C) Youngsuk Heo}

All rights reserved

2008 


\section{Dedication}

This dissertation is dedicated to my father in celebration of his $70^{\text {th }}$ birthday. 


\section{Acknowledgements}

I would like to first show my great appreciation to Prof. Ronald G. Larson during the whole Ph.D. years for being a great advisor, mentor, leader, and moderator. Since I joined his research group in the fall of 2002, he has constantly disciplined and encouraged me in not only doing research, but also showing leadership, brutal honesty in communication with colleagues, and even balancing work responsibilities with family obligations. He has always inspired me to first watch the real world rather than to be initially trapped in mathematical equations so that I can identify the real problems and find a solution to it. He has been very understanding and trying to give me as many opportunities as possible even when I got stuck in my research and struggled to get out of it.

I also appreciate the time and effort of my doctoral committee members, Prof. Peter F. Green, Prof. David C. Martin, and Prof. Michael J. Solomon.

I would like to give my special thanks to former and current Larson group members for being supportive and enthusiastic in discussing research and life. I am particularly indebted to Seung Joon, Sachin, and Richard in starting my doctoral research on the rheology of complex fluids. They have provided me with rich and knowledgeable background, and methodologies I needed to take in this field of research. I was also very lucky to interact and communicate with Anshuman, Qiang, and Zuowei, who are all 
dedicated to learning new things, and helping other people with what they uniquely could do. I thank Tyson, Ji Hoon, Weixian, and Hwankyu, who joined the Larson group at the same year, for being friends indeed for the past five years in taking classes, and making research progress around the same time.

Siva deserves my profound thanks for influencing me to explore and pursue food industry career by utilizing my specialty in rheology. Prof. Leslie A. Olsen and Deborah Van Hoewyk at the Technical Communications Program greatly helped me in preparing resume, cover letter, and presentation for job seeking. Without their help, I would not have taken this big step to my next field, food science and engineering.

I could not forget about the wonderful help that I have received from Nonna even before I officially joined the Macromolecular Science and Engineering Program. When my wife miscarried just a week after we arrived in Ann Arbor, she comforted us and gave a big hand to us in many meaningful ways. She has been playing the role of mom while my wife and I have been far away from home as she asked us to call.

Most importantly, I would like to thank my family: my wife, parents, brother, sister, inlaws, and my two sons, Nathan and Caleb. They have been the biggest motivation I have kept so far. Especially, my wife, So Young, has been and will be without a doubt my greatest supporter and buddy for my life. 


\section{Table of Contents}

Dedication.....................................................................

Acknowledgements..............................................................

List of Figures............................................................

List of Tables..............................................................

Chapter

1. Introduction............................................................

1.1. Analytical Rheology for Polymer Melts..................................

1.2. Analytical Rheology for Semidilute Polymer Solutions.......................

1.3. Universal Scaling of Semidilute Polymer Solutions...........................6

2. The Scaling of Zero-shear Viscosities of

Semidiute Polymer Solutions with Concentration ...............................10

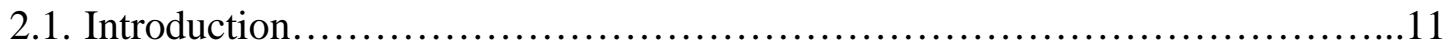

2.2. Experimental Data................................................. 14

2.2.1. Sample Preparation.............................................14

2.2.2. Rheometry................................................. 15

2.3. Results and Discussion............................................18

2.3.1. Determining the Zero-shear Viscosity.............................18

2.3.2. The Zero-shear Viscosities of $\lambda$-DNA Solutions and the Universal Dependence on Concentration.........................20

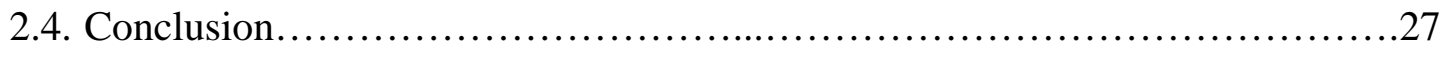


3. Universal Scaling of Linear and Nonlinear Rheological Properties of Semidilute and Concentrated Polymer Solutions...................................32

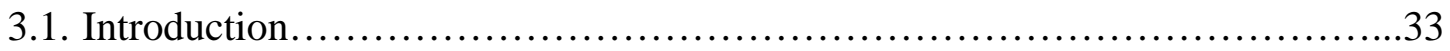

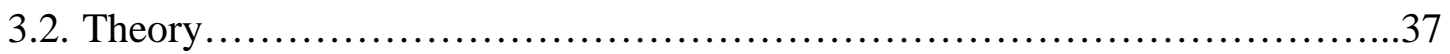

3.3. Experimental Materials and Methods........................................43

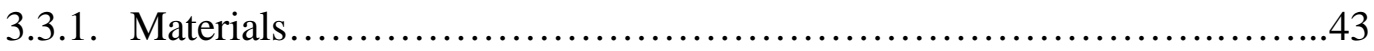

3.3.2. Sample Preparation...............................................44

3.3.2.1.Weight-average Molecular Weight Measurements......................44

3.3.2.2. Preparation of Solutions..........................................45

3.3.2.3. Measuring Rheological Properties with Bubble-free Samples..........46

3.4. Results and Discussion................................................47

3.4.1. Nearly Monodisperse Polystyrene Solutions...........................47

3.4.2. Melt vs. Solutions Rheology ........................................56

3.4.2.1.Nearly Monodisperse 1,4 Polybutadiene Melts and Solutions in Phenyl Octane...................................................................59

3.4.3. Extension to Binary Blends....................................................................67

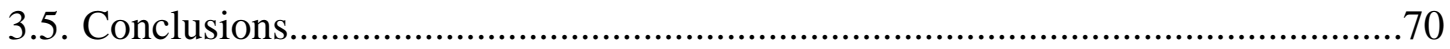

4. Semidilute Solution Rheology for Turbulent Drag Reduction Phenomenology.........76

4.1. Introduction....................................................................................................77

4.2. Sample Preparations.........................................................................................79

4.3. Steady Shear Viscosity Measurements....................................................................79

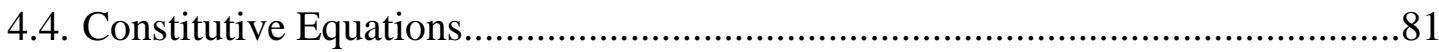

4.4.1. FENE-P Fitting with Simulated Annealing...............................................81

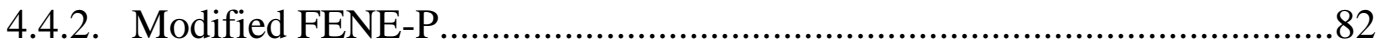




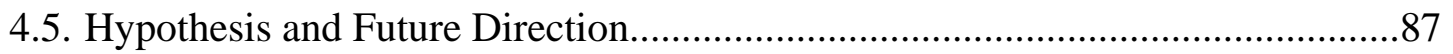

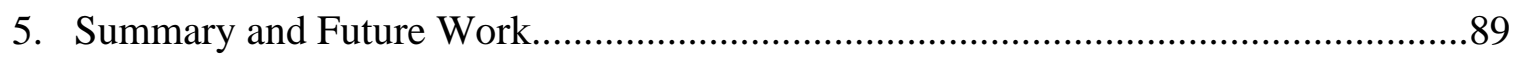

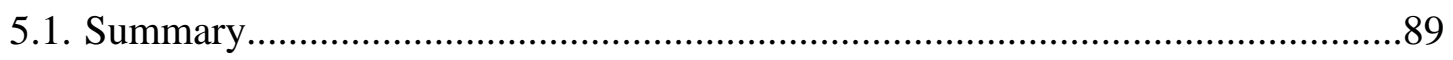

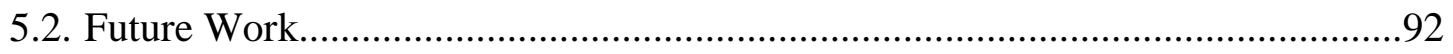

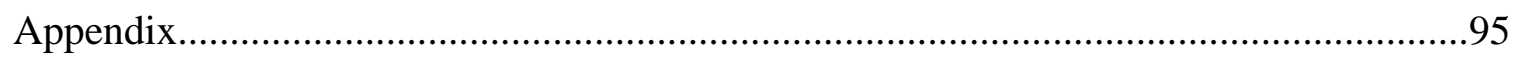




\section{List of Figures}

\section{Chapter 1}

Figure 1.1. Normalized intrinsic viscosity $[\eta] /[\eta]_{0}$ versus Deborah number polystyrene (PS) solutions in decalin and toluene. ${ }^{3}$ The numbers in the parentheses are the molecular weight of PS, and M stands for million $\mathrm{g} / \mathrm{mol}$.............................................2

Figure 1.2. Storage and loss moduli of polybutadiene melts with different chain architectures. Dots and lines respectively represent experimental data and theoretical predictions. $^{4,5}$ (b) $M_{a}$ and $M_{b}$ respectively stand for the molecular weight of the arm and backbone of the "comb" polymer.

.3

Figure 1.3. Schematic of particle dynamics in a viscoelastic medium. $a$ and $\xi$ respectively represent the diameter of a colloid particle, and the correlation length, or the mesh size of the polymer solution ${ }^{7}$.

Figure 1.4. Classification of polymer solutions according to the extent of interactions of polymer chains ${ }^{8}$....

Figure 1.5. A schematic diagram of the polymer chain length scale with its number of monomers in a very good solvent $(v=0.588) . R, \xi, l_{s}$, and $b_{K}$ respectively represent the end-to-end distance, the correlation blob size, above which excluded volume interaction and hydrodynamic interactions are cancelled out, the swelling length, a length scale below which thermal fluctuation dominates over excluded volume interaction, and the Kuhn length. .7

\section{Chapter 2}

Figure 2.1. Classification of polymer solutions in terms of concentration and molecular weight ${ }^{1}$

Figure 2.2. Schematic diagram of Contraves Low Shear 30............................................16 
Figure 2.3. Dependence of shear viscosity on shear rate for $\lambda$-DNA solutions for severalconcentrations at $25.0 \pm 0.3^{\circ} \mathrm{C}$. The lines are the fits of the Carreau-Yasuda model with parameters of the fit given in Table 2.1. .18

Figure 2.4. Master scaling curve of normalized zero shear viscosities of polymer solutions against normalized concentrations. $\mathrm{M}$ stands for the weight-averaged molecular weight, $\mathrm{C}$ for degrees Celsius, TE for Tris-EDTA, PEO for poly (ethylene oxide), PS for polystyrene, TCP for tricresyl phosphate, PI for polyisoprene, and PB for polybutadiene. $\eta_{p}$ is the polymer contribution to zero shear viscosity, $\eta_{\text {Rouse }}$ is the hypothetical Rouse viscosity, and $c_{e}$ is the entanglement concentration. See text for detail. .26

\section{Chapter 3}

Figure 3.1. Osmotic pressure for polystyrene (PS) in toluene at $25^{\circ} \mathrm{C}$ (a good solvent) and in cyclohexane at the $\Theta$ temperature, $35^{\circ} \mathrm{C}$. Solid lines denote experimental data of PS/toluene ${ }^{22}$, and dashed lines denote those of PS/cyclohexane.

Figure 3.2. Plateau modulus against volume fraction of nearly monodisperse polystyrene solutions in Aroclor $1248^{25,26}$ (open diamonds and open triangles), n-butyl benzyl phthalate $^{23}$ (open squares), and in tricresyl phosphate ${ }^{24}$ (filled circles). The line is a leastsquares fit to all data. .41

Figure 3.3. Sample Zimm plot of $1.28 \times 10^{6} \mathrm{~g} / \mathrm{mol}$ polystyrene in toluene at room temperature. Here, $\theta, c, R(\theta)$, and $K$ respectively denote the detection angle, the concentration of a sample in $\mathrm{g} / \mathrm{ml}$, the excess Rayleigh ratio, and $K=\frac{4 \pi^{2}}{N_{\mathrm{A}}} \frac{n_{0}^{2}}{\lambda_{0}^{4}}\left(\frac{d n}{d c}\right)^{2}$ with $N_{A}$ the Avogadro's number, $n_{0}$ the solvent refractive index, $\lambda_{0}$ the vacuum wavelength of incident light, and $d n / d c$ the refractive index increment. Note that a negative "stretch factor," -2230, has been used for enhanced interpretability. We employed a "Berry plot" for $2.53 \times 10^{6} \mathrm{~g} / \mathrm{mol}$ and $6.62 \times 10^{6} \mathrm{~g} / \mathrm{mol}$ polystyrene, since this method of plotting is recommended for polymers with the molecular weight higher than 1 million $\mathrm{g} / \mathrm{mol}$........45

Figure 3.4. Plots testing "universal scaling" of the linear viscoelastic properties of semidilute monodisperse polystyrene (PS)/tricresyl phosphate (TCP) solutions. In each plot, normalized storage and loss moduli, $G^{\prime} / G_{N}^{0}$ and $G^{\prime \prime} / G_{N}^{0}$ are plotted against normalized frequency, $\omega \tau_{e, \text { scaling }}$ at a given nominal $c / c_{e}$. The small differences in values of $c / c_{e}$ within each figure are recorded in the legends. Filled circles, open squares, and open circles respectively represent the storage and loss moduli of $1.28 \times 10^{6} \mathrm{~g} / \mathrm{mol}$, $2.53 \times 10^{6} \mathrm{~g} / \mathrm{mol}$, and $6.62 \times 10^{6} \mathrm{~g} / \mathrm{mol}$ PS solutions. .50

Figure 3.5. "Universal scaling" of the nonlinear viscoelastic properties of semidilute monodisperse PS/TCP solutions. The normalized first normal stress coefficient 
$\psi_{1} /\left(\eta_{\text {Rouse }} \tau_{e, \text { scaling }}\right)$ and the normalized steady shear viscosity $\eta_{p} / \eta_{\text {Rouse }}$ are plotted against the normalized shear rate, $\dot{x}_{e, \text { scaling }}$, at two nominal values of $c / c_{e}$, where the Rouse viscosities for these two concentrations are computed from $\eta_{\text {Rouse }}=\eta_{s}\left(c[\eta]_{0}\right)^{1 /(3 v-1)}$ as given earlier in this section, and are tabulated in Table 3.4. Filled and open diamonds, respectively, represent the normalized first normal stress difference coefficients of $6.62 \times 10^{6} \mathrm{~g} / \mathrm{mol}$ and $2.53 \times 10^{6} \mathrm{~g} / \mathrm{mol}$ PS solutions. Filled and open circles, respectively, represent the normalized steady shear viscosities of $6.62 \times 10^{6} \mathrm{~g} / \mathrm{mol}$ and $2.53 \times 10^{6} \mathrm{~g} / \mathrm{mol}$ PS solutions. .53

Figure 3.6. Schematic of the scaling of plateau modulus $G_{N}^{0}(\phi)$ of polymer solutions with three different excluded volume exponents, namely $v=0.57,0.53$, and 0.51 , versus volume fraction $\phi$. See text for detail. .59

Figure 3.7. Normalized complex moduli, $G^{\prime} / G_{N}^{0}$ and $G^{\prime \prime} / G_{N}^{0}$ against normalized frequency, $\omega \tau_{e, D E}$, for nearly monodisperse polybutadiene (PBd) melts and solutions. Here, $\tau_{e, D E}$ for PBd solutions is computed from $\tau_{e, D E}=K_{1} \cdot \tau_{e, \text { scaling }}, K_{1}=6.52$, and Eq. (20), with $\alpha_{c}=1.29$, and $N_{e}(1)=35.7$ (see Table 3.5). Filled symbols denote $G^{\prime} / G_{N}^{0}$ and $G^{\prime \prime} / G_{N}^{0}$ for PBd melts with $M_{w}=9.25 \times 10^{5}, 2.01 \times 10^{5}, 0.97 \times 10^{5}, 0.44 \times 10^{5}$, and $0.21 \times 10^{5}$ $\mathrm{g} / \mathrm{mol},{ }^{36}$ and open symbols denote PBd solutions with $M_{w}=9.25 \times 10^{5} \mathrm{~g} / \mathrm{mol}$ at volume fractions of $0.488,0.280,0.140,0.062,0.027$, and $0.021 .^{5}$. .64

Figure 3.8. The reduced crossover relaxation time of polybutadiene (PBd) melts and $\mathrm{PBd} /$ phenyl octane $(\mathrm{PhO})$ solutions versus the number of entanglements per molecule: $N / N_{e}$ for melts and solutions. Here, we employ $M_{e}(1)=1.93 \times 10^{3} \mathrm{~g} / \mathrm{mol}$ from Likhtman and McLeish ${ }^{28}$ to obtain $N_{e}(1)=M_{e}(1) / M_{0}$ with $M_{0}=54 \mathrm{~g} / \mathrm{mol}$ the monomer molecular weight of PBd. For solutions, we use the scaling law $N_{e}(\phi)=N_{e}(1) \phi^{-\alpha_{c}}=\left(1.93 \times 10^{3} / 54\right) \phi^{-}$ 1.29. Filled circles represent PBd melts $\left(N / N_{e}=10.7,22.9,50.3,104.2\right.$, and 479.3) and open diamonds represent $\mathrm{PBd} / \mathrm{PhO}$ solutions of $M_{w}=9.25 \times 10^{5}\left(N / N_{e}=3.4,4.6,13.3\right.$, 37.9, 92.8, 184.1, and 479.3)...... .66

Figure 3.9. Superposition of linear viscoelastic properties of two binary blends of $\mathrm{PS} / \mathrm{TCP}$ solutions at six different values of $c / c_{e}$ : (a) $c / c_{e}=0.50$, (b) $c / c_{e}=1.00$, (c) $c / c_{e}=1.50$, (d) $c / c_{e}=2.00$, (e) $c / c_{e}=2.50$, and (f) $c / c_{e}=3.00$. Filled circles and diamonds respectively represent the normalized storage and loss moduli of the solutions with equal mass fractions of $M_{w, \text { short }}=1.28 \times 10^{6} \mathrm{~g} / \mathrm{mol}$ and $M_{w, \text { long }}=2.68 \times 10^{6} \mathrm{~g} / \mathrm{mol}$. Open circles and diamonds respectively represent the normalized storage and loss moduli of the solutions with $M_{w, \text { short }}=2.68 \times 10^{6} \mathrm{~g} / \mathrm{mol}$ and $M_{w, \text { long }}=5.56 \times 10^{6} \mathrm{~g} / \mathrm{mol} . \tau_{e, \text { scaling }}$ is based on the weight average molecular weight of long and short chains. .68

Figure 3.10. The same as Figure 3.9 except for nonlinear viscoelastic properties: (a) $c / c_{e}=0.50$, and (b) $c / c_{e}=1.00$.. .70 


\section{Chapter 4}

Figure 4.1. Schematic of the concentration effect on the relative drag reduction to Newtonian flow. Beyond the onset of drag reduction in Reynolds number, polymer solutions with higher concentration will reach the maximum drag reduction more quickly

Figure 4.2. Shear-dependent viscosities of WSR301 PEO solutions at four concentrations with three different rheometers. The concentrations shown next to the rheometer used in the legend correspond to the shear-dependent viscosities from top to bottom. .80

Figure 4.3. FENE-P fitting (dashed lines) with $b$ and $\eta_{s}$ fixed to the combined experimental data of WSR 301. Note that we were not able to acquire the FENE-P fit to $4000 \mathrm{ppm}$ data due to the lack of sufficient plateau region. .81

Figure 4.4. Experimental data points of semidilute polymer solutions in good solvents lying on a single universal plot, $\eta_{p} / \eta_{\text {Rouse }}=(43 \pm 2) \times\left(c / c_{e}\right)^{3.12 \pm 0.05}$ .84

Figure 4.5. Comparison Non-aggregated PEO $\left(M_{w}=3.33 \times 10^{5}\right)$ solutions with two different entanglement molecular weights, $M_{e}=4400 \mathrm{~g} / \mathrm{mol}$ (black filled circles) and $M_{e}=1624 \mathrm{~g} / \mathrm{mol}$ (gray filled circles). 85

Figure 4.6. Comparison of zero-shear viscosities (black filled symbols) and "solvent viscosities" (gray filled symbols) of WSR 301 (circles), 308 (diamonds), and N60K (triangles) on the universal curve. .86

Figure 4.7. Modified FENE-P fitting by utilizing Eqs. 3-6 .86

\section{Chapter 5}

Figure 5.1. Dimension of a polymer chain in solution according to the quality of solvent used; see text. ${ }^{1}$

\section{Appendix}

Figure A.1. Normalized loss modulus versus normalized frequency of PS melts and PS/TCP solutions with $\phi_{s}=0.09$. Filled and open symbols respectively represent melts and solutions. 
Figure A.2. Normalized loss modulus versus normalized frequency of PS melts and PS/TCP solutions with $\phi_{s}=0.12$. Filled and open symbols respectively represent melts

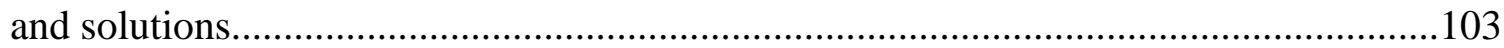

Figure A.3. Normalized loss modulus versus normalized frequency of PS melts and PS/TCP solutions with $\phi_{s}=0.15$. Filled and open symbols respectively represent melts and solutions. 


\section{List of Tables}

\section{Chapter 2}

Table 2.1. The parameter values of the Carreau-Yasuda curvefit to shear thinning curve of $\lambda$-DNA solution for various concentrations. For the concentration $\mathrm{c}=0.28 \mathrm{~g} / \mathrm{L}$, the data in the shear rate range $0.20-0.38 \mathrm{~s}^{-1}$ were omitted when fitting the parameters, since the parameters were highly sensitive to slight noise in the these few data point; see discussion in text................................................................................................................20

Table 2.2. Scaling parameters for various polymer-solvent pairs at various temperatures .24

\section{Chapter 3}

Table 3.1. Nominal and actual molecular weights of polystyrene samples. .44

Table 3.2. The parameters of polystyrene (PS)/tricresyl phosphate (TCP) solutions at $25^{\circ} \mathrm{C}$; the zero-shear viscosity of TCP $\eta_{s}$, the number of correlation blobs per entanglement for PS/TCP solutions $n_{e}$, the excluded volume exponent for PS/TCP solutions $v$, the dilution exponent $\alpha$ for PS/TCP solutions, the plateau modulus of PS melt at $180^{\circ} \mathrm{C} G_{N}^{0}(1)$, and the densities of PS and TCP at $25^{\circ} \mathrm{C}, \rho_{P S}$ and $\rho_{T C P} \ldots \ldots . . . . . . . .47$

Table 3.3. Parameters of polystyrene (PS) of three different molecular weights in tricresyl phosphate (TCP): the weight-average molecular weight $M_{w}$, the intrinsic viscosity $[\eta]_{0}$, the radius of gyration $R_{g}$, the overlap concentration $c^{*}$, the second virial coefficient multiplied by the molecular weight $M_{w} A_{2}$, and the entanglement concentration $c_{e}$ of each PS sample. .48

Table 3.4. Volume fraction $\phi$, equilibration time $\tau_{e \text {,scaling }}$ calculated from Eq. (12), and the plateau modulus $G_{N}^{0}(\phi)=G_{N}^{0}(1) \phi^{1+\alpha}$ for each PS/TCP solution at various molecular weights $M_{w}$, and reduced concentrations $c / c_{e}$

Table 3.5. The equilibration times and the plateau moduli of a series of $\mathrm{PBd} / \mathrm{PhO}$ solutions $\left(M_{w}=9.25 \times 10^{5} \mathrm{~g} / \mathrm{mol}\right)$. Here, we employ $N_{e}(\phi)=N_{e}(1) \phi^{-\alpha_{c}}$ to compute the number of monomers per entanglement at a volume fraction $\phi$ 
Table 3.6. Weight-average molecular weights and parameters of two binary blends of PS

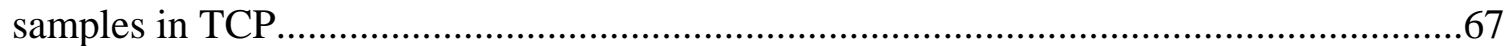

\section{Appendix}

Table A.1. Parameters values for calculating Eqs. (1)-(4)...........................................97

Table A.2. Entanglement density and normalizing parameters depending on different swelling volume fraction.. 


\section{Chapter 1}

\section{Introduction}

\section{1. Analytical Rheology for Polymer Melts}

The molecular nature of polymers, which consist of long chains of covalently bonded atoms, was first introduced by Staudinger in the 1920s. Prior to his work, polymers were generally understood as aggregates of "colloids", and it took more than a decade for the scientific community to widely accept this concept. With this change in perspective on polymers and their growing industrial importance along with the World War II, many researchers became interested in how the molecular structure of a polymer affects its flow behavior when processed in melt state. ${ }^{1,2}$

Dilute polymer solutions, in parallel with melts, have been also extensively studied for the purpose of characterizing the chain dimensions, architectures, and dynamics of an isolated single polymer chain in solution at different length scales by using many different analytical methods such as osmometry, light scattering, size exclusion chromatography, NMR, IR, and rheometry. Figure 1.1 illustrates how the conformation of a polymer chain in a dilute solution under shear flow strongly affects its sheardependent rheology. Polystyrene (PS) solutions with $M_{w}=13.6$ million $\mathrm{g} / \mathrm{mol}$ in two different solvents, decalin $(\Theta$ solvent) and toluene (good solvent), show a drastic difference in shear thinning behavior as a polymer chain in a good solvent is very 
sensitive to hydrodynamic interaction through shear flow while the one in a $\Theta$ solvent is not, due to its free-draining effect.

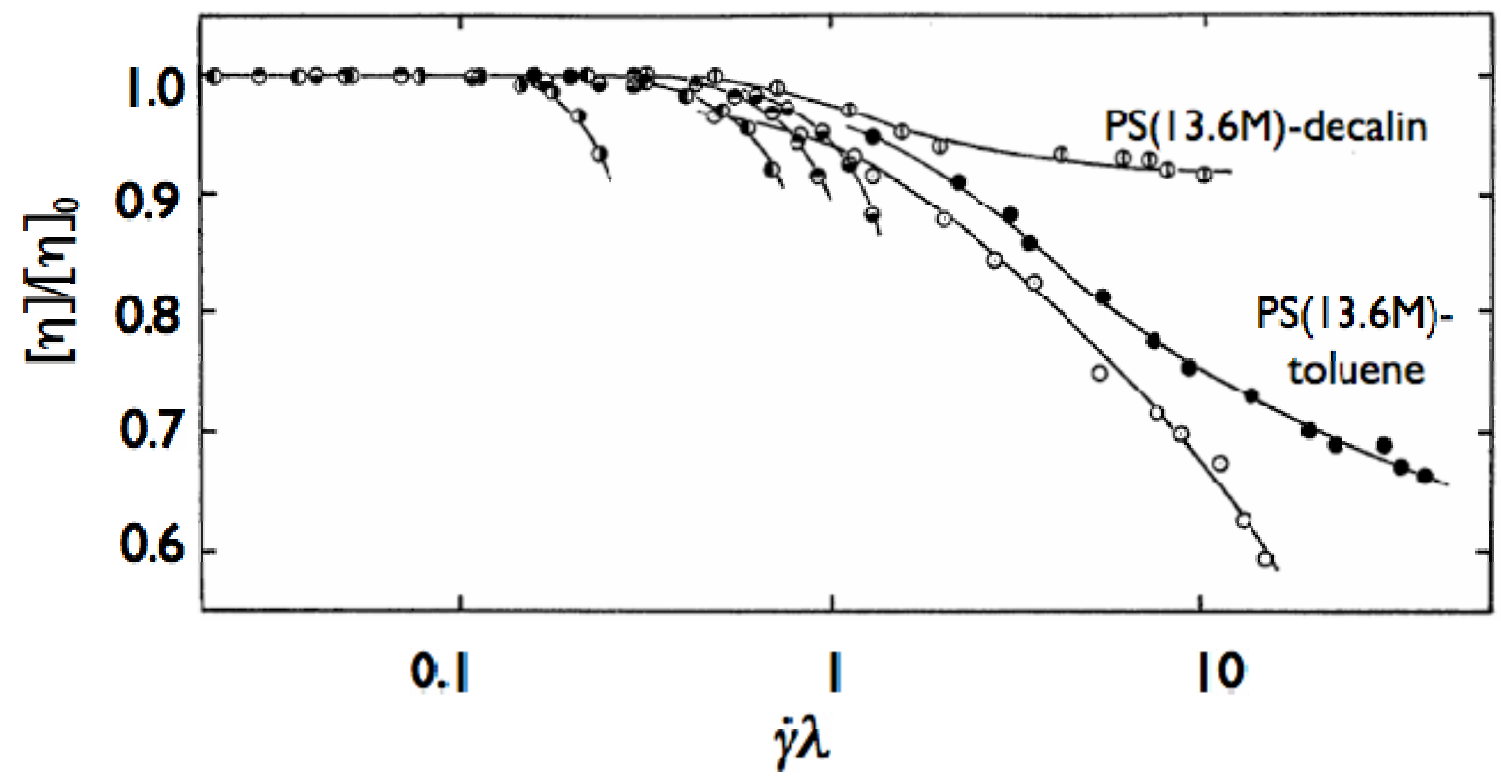

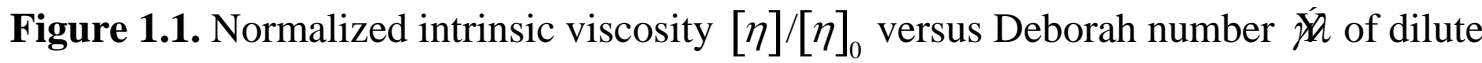
polystyrene (PS) solutions in decalin and toluene. ${ }^{3}$ The numbers in the parentheses are the molecular weight of PS, and M stands for million $\mathrm{g} / \mathrm{mol}$.

In addition to characterizing the structure and rheology of a given polymer, single-site metallocence catalysts, which contain a metal-carbon site where an olefin can insert itself for addition polymerization, makes possible to control the molecular structure of a polymer. ${ }^{1}$ Thus, we can not only predict the flow properties of a polymer with a given molecular structure, but also control the molecular structure of a polymer to obtain desired flow properties. Furthermore, we can check whether the synthesis has been successful. This is called analytical rheology, and it gives us a solid basis for constructing a relationship of molecular structure-flow property as shown below in Figure 1.2. Each of these figures represents the storage and loss moduli of a molten polymer with a different 
molecular structure, such as linear and comb. These polymers are synthesized with single-site metallocene catalysts, and the molecular structures are checked to see whether the desired structure has been created. These plots show that (1) storage and loss moduli reveal the molecular structure of a polymer, and (2) rheology can be used as an easy and cost-effective analytical tool for probing the molecular structure as well as other spectroscopic methods.

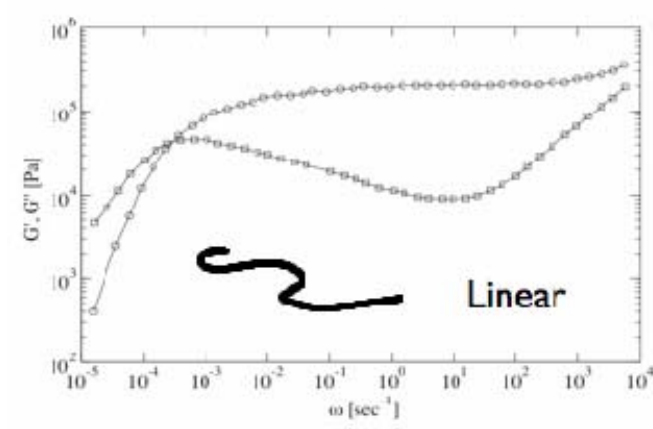

(a) polybutadiene ( $\left.M_{w}=201 \mathrm{~kg} / \mathrm{mol}\right)$

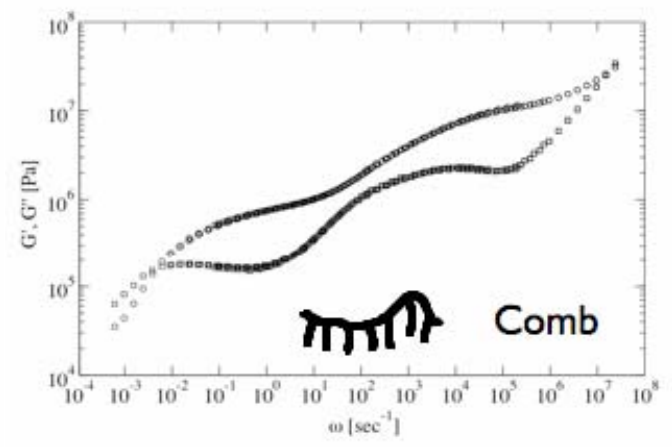

(b) polybutadiene ( $M_{a}=10.3 \mathrm{k}, M_{b}=123 \mathrm{k} \mathrm{g} / \mathrm{mol}$ )

Figure 1.2. Storage and loss moduli of polybutadiene melts with different chain architectures. Dots and lines respectively represent experimental data and theoretical predictions. ${ }^{4,5}$ (b) $M_{a}$ and $M_{b}$ respectively stand for the molecular weight of the arm and backbone of the "comb" polymer.

\subsection{Analytical Rheology for Semidilute Polymer Solutions}

Polymer melts rheology has been playing a main role in polymer processing with the development of plastics industry for the past decades. However, the rheology of polymer chains in non-dilute solutions has not been studied as extensively as polymer melts, despite their ubiquity and commercial importance in chemical and materials processing. In fact, the research on liquid has not progressed as much as that on solid and gas, because the first experimental methods and devices were developed to interpret the structure of these two states. In addition, although solid and gas, which are at two 
opposite extremes in the molecular order, were extensively investigated by theory and mathematics, liquids represent a compromise between order and disorder and have hampered a comprehensive theoretical treatment. ${ }^{6}$ In the same way, semidilute solutions, which reside in the middle of concentration spectrum, needs a good compromise between two extremes-melts and dilute solutions by elucidating the underlying physics.

Here are some examples of materials and studies where polymers in non-dilute solutions play main roles in forming and maintaining the desired viscoelastic properties of the systems.

- Dough: gluten, a mixture of two proteins, gliadin and glutenin, is responsible for the elasticity of kneaded dough. Wheat flour with high gluten content is used for breads, whereas flour with lower gluten content is used for cakes; composition of high molecular weight polymer dictates the texture.

- Hemorheology: blood plasma is the largest single component of blood making up more than a half of total blood volume, and its protein content is necessary to hold the serum within the vessels.

- Inks: in modern inkjet printing, jets and droplets are formed at extremely high speeds with the liquids experiencing very high shear rates. The fluids contain significant amount of polymer and/or particulates having complex rheological properties.

- Biophysics: semiflxible polymers form cytoskeleton, a network of dynamic structures that give animal cells mechanical integrity. Thus, the viscoelastic behavior of F-actin, a major component of these proteins, has been experimentally investigated using microrheology such as dynamic light scattering, diffusing wave 
spectroscopy, and video particle-tracking. This research, in turn, has drawn significant attention on the links between cytoskeleton and mechanical models of entangled rods.

- Microrheology: along the same line with cytoskeleton, linear viscoelastic behavior of colloidal particles dispersed in polymer solutions, a representative example of viscoelastic media, is crucial to understanding suspensions of particles; see Figure 1.3. ${ }^{7}$

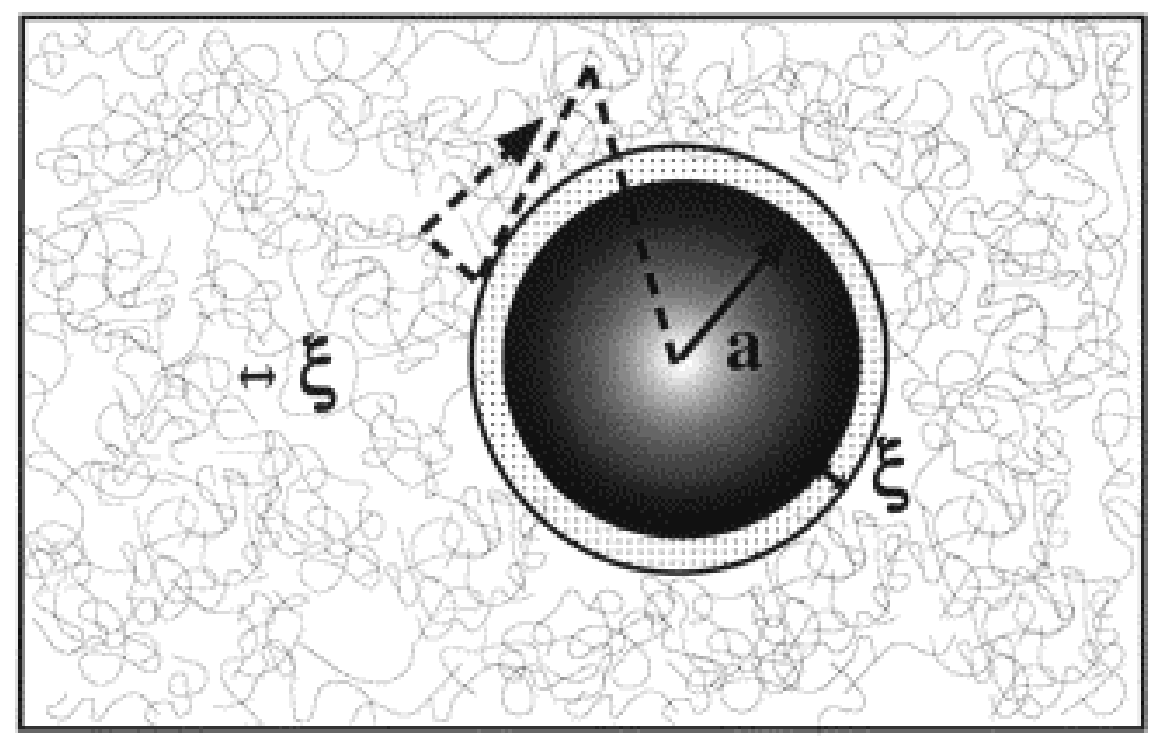

Figure 1.3. Schematic of particle dynamics in a viscoelastic medium. $a$ and $\xi$ respectively represent the diameter of a colloid particle, and the correlation length, or the mesh size of the polymer solution. ${ }^{7}$

Non-dilute solutions are broken down into two different regimes according to the chain dimension of polymers: semidilute and concentrated solutions. Since the chain dimension of polymers in concentrated regime is identical to that of melts, we are going to have more focus on semidilute solutions in this dissertation. As shown in Figure 1.4, semidilute solutions are defined as the solutions of which concentrations exceed the 
overlap concentration $c^{*}$ as polymer chains start overlapping and interacting with each other.
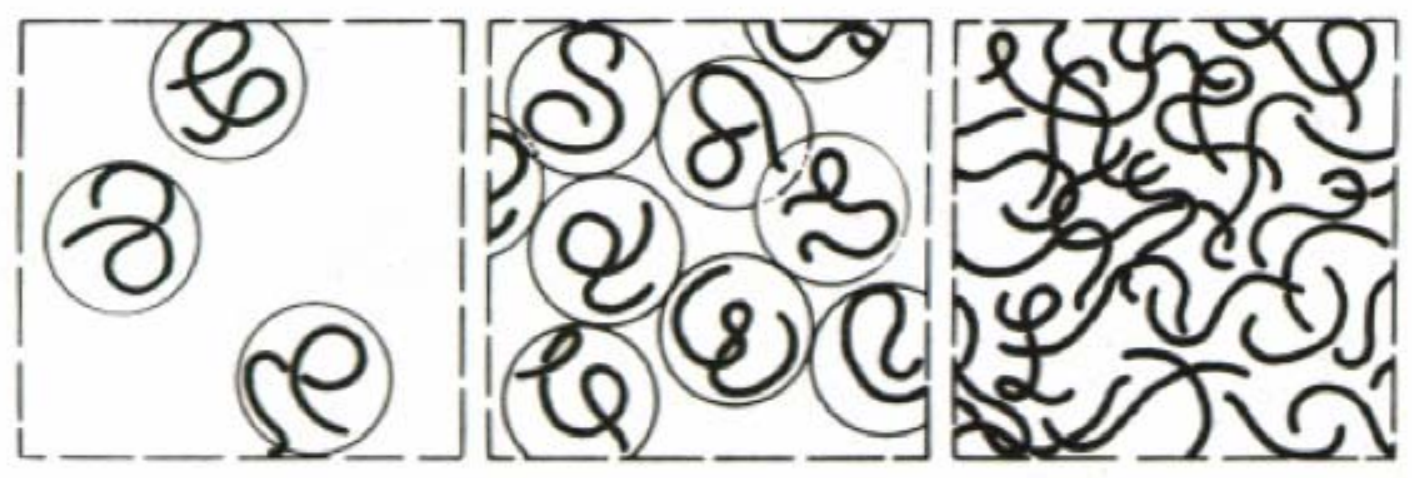
(a) $c<c^{*}$
(b) $c \cong C^{*}$
(c) $C^{*}<C$

Figure 1.4. Classification of polymer solutions according to the extent of interactions of polymer chains. ${ }^{8}$

The biggest challenge in dealing with semidilute polymer solutions compared to polymer melts or dilute solutions is that we need to consider both polymer-solvent interaction and polymer-polymer interaction simultaneously while there exists only one kind of interaction for both polymer melts and dilute polymer solutions: polymerpolymer interaction for melts, and polymer-solvent interaction for dilute solutions. In other words, polymer chains in solution interact thermodynamically through their interaction potential, and hydrodynamically through flows mediated by solvent. ${ }^{7}$ De Gennes' blob model is employed to construct the universal scaling of rheological properties of polymer solutions based on the relationship of polymer chain size with its length summarized in Figure 1.5. More detail will be covered in the following chapters. 


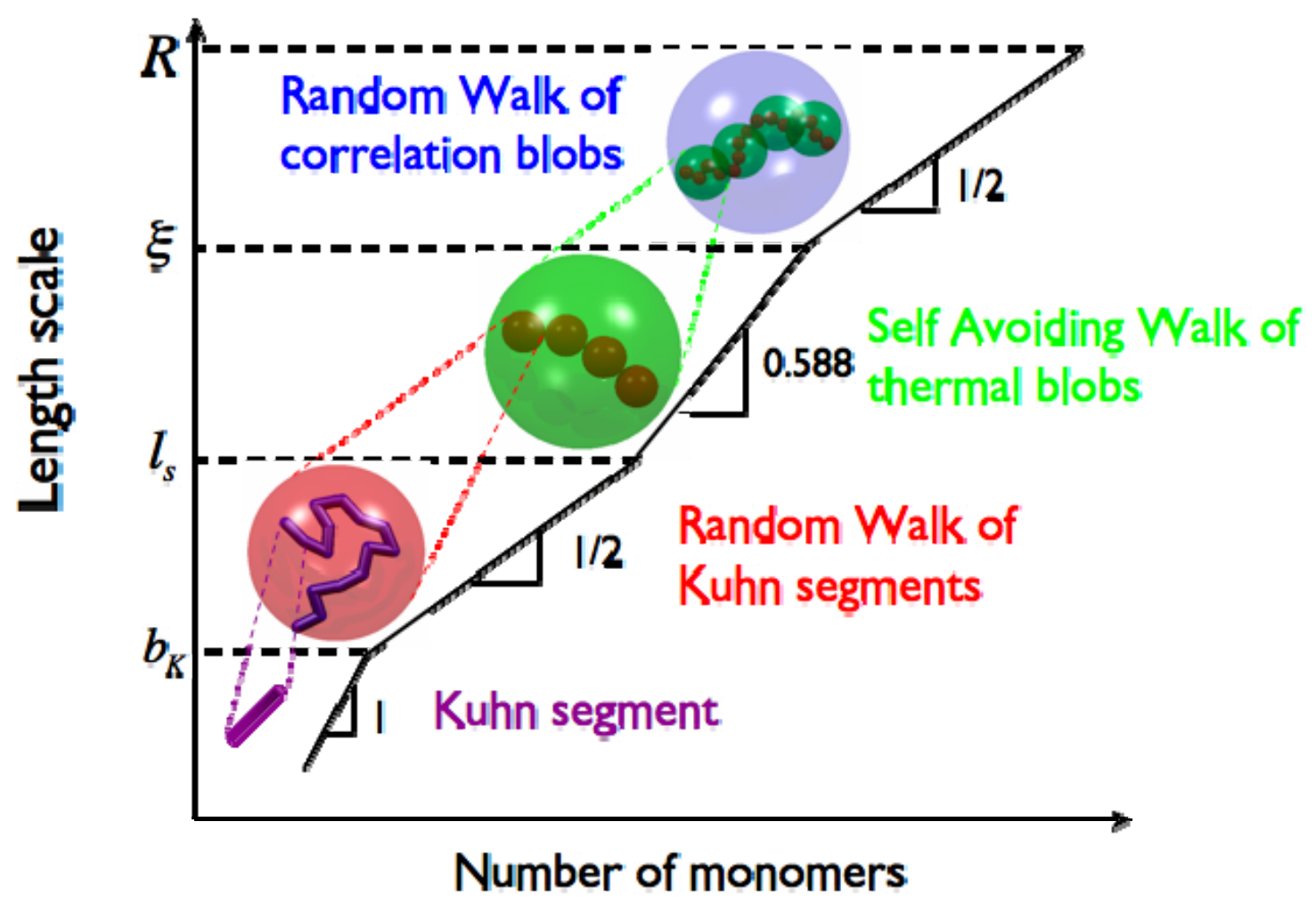

Figure 1.5. A schematic diagram of the polymer chain length scale with its number of monomers in a very good solvent $(v=0.588) . R, \xi, l_{s}$, and $b_{K}$ respectively represent the end-to-end distance, the correlation blob size, above which excluded volume interaction and hydrodynamic interactions are cancelled out, the swelling length, a length scale below which thermal fluctuation dominates over excluded volume interaction, and the Kuhn length.

\subsection{Universal Scaling of Polymer Solutions}

Chemically identical polymers with the same molecular weight but different topologies often have completely different rheology. Conversely, chemically different polymers with similar topologies show similar diffusive and/or convective motions upon stress/strain. ${ }^{9}$ This is called dynamic similarity, and has been demonstrated for polymer melts and colloidal aggregations. ${ }^{10}$ By using this concept, we can obtain a physical insight into some seemingly different systems. For example, the Himalayas arose from the Indian plains as sub-continents of India and Asia collided, which is analogous to piling-up of peanut butter by spreading knife. Even though the viscosity and time scales 
are quite different, the essential geometry and dynamics can be considered the same. This is the basis of universal scaling of polymeric liquids under stress or strain; once the number of thermodynamically and hydrodynamically independent units, or correlation blobs, are determined for a polymer chain in semidilute solution, we should be able to predict the rheological properties of this semidilute solution based on the comparison with polymer melts having the same number of monomers. The following chapters will investigate this hypothesis in detail. 


\section{References}

1. Dealy, J. M.; Larson, R. G. Structure and rheology of molten polymers: Hanser Publishers: Cincinnati, 2006.

2. Dealy, J. M; Wissbrun, K. F. Melt rheology and its role in plastics processing: Van Nostrand Reinhold: New York, 1990.

3. Noda, I.; Yamada, Y.; Nagasawa, M. J. Phys. Chem. 1968, 72, 2890-\&.

4. Baumgaertel, M.; Derisa, M. E.; Machado, J.; Masse, M.; Winter, H. H. Rheo. Acta 1992, 31, 75-82.

5. Daniels, D. R.; McLeish, T. C. B. Macromolecules 2001, 34, 7025-7033.

6. Rohn, C. L. Analytical Polymer Rheology: Hanser/Gardener, Munich, 1995.

7. Solomon, M. J.; Lu, Q. Curr. Op. in Colloid \& Interface Sci. 2001, 6, 430-437.

8. de Gennes, P. -G. Scaling Concepts in Polymer Physics; Cornel University Press: Ithaca, New York, 1979.

9. Graham, R. S. Ph. D. Thesis; University of Leeds, 2002

10. Lin, M. Y.; Lindsay, H. M.; Weitz, D. A.; Ball, R. C.; Klein, R.; Meakin, P. Nature 1989, 339, 360-362 


\title{
Chapter 2
}

\section{The Scaling of Zero-shear Viscosities of Semidilute Polymer Solutions with Concentration}

\begin{abstract}
To test the universality of the dependence of zero-shear viscosity on concentration for both flexible and locally semiflexible polymers in good solvents, we collected multiple literature data sets and measured the zero shear viscosity of $\lambda$-phage DNA over a range of semidilute concentrations. We found that all experimental data above a critical concentration $c / c_{e}>0.5$ fall on a single empirical curve given by $\eta_{p} / \eta_{\text {Rouse }}=(43 \pm 2) \times\left(c / c_{e}\right)^{3.12 \pm 0.05}$ and this scaling law is in good agreement with the theoretical one, $\eta_{p} / \eta_{\text {Rouse }} \approx\left(c / c_{e}\right)^{2.4 /(3 v-1)}$ with $v$ the excluded volume exponent, $\eta_{p}=\eta_{0}-\eta_{s}$ the polymer contribution to the zero shear viscosity of the solution with $\eta_{0}$ the zero-shear viscosity and $\eta_{s}$ the solvent viscosity, $\eta_{\text {Rouse }}$ the hypothetical Rouse polymer viscosity, and $c_{e}$ the entanglement concentration of the polymer solution. ${ }^{8,12,13}$ This scaling law provides a basis for estimating viscosities for arbitrary semidilute entangled polymer solutions from a knowledge of the solvent viscosity, the entanglement molecular weight in the melt, the excluded volume exponent, the second virial coefficient, and the intrinsic viscosity.
\end{abstract}




\subsection{Introduction}

Graessley $^{1}$ classified polymer solutions into five different regimes according to concentration and molecular weight; see Figure 2.1. There are well-established rheological theories for three of these regimes, namely the Zimm model for dilute solutions, the Rouse model for concentrated but not entangled solutions, and the DoiEdwards model for concentrated entangled solutions and melts. Although not strictly appropriate, the other two regimes have been described by applying the Rouse model to semidilute unentangled solutions ${ }^{3-9}$ and the Doi-Edwards model to semidilute entangled solutions. ${ }^{3,4,6-9}$

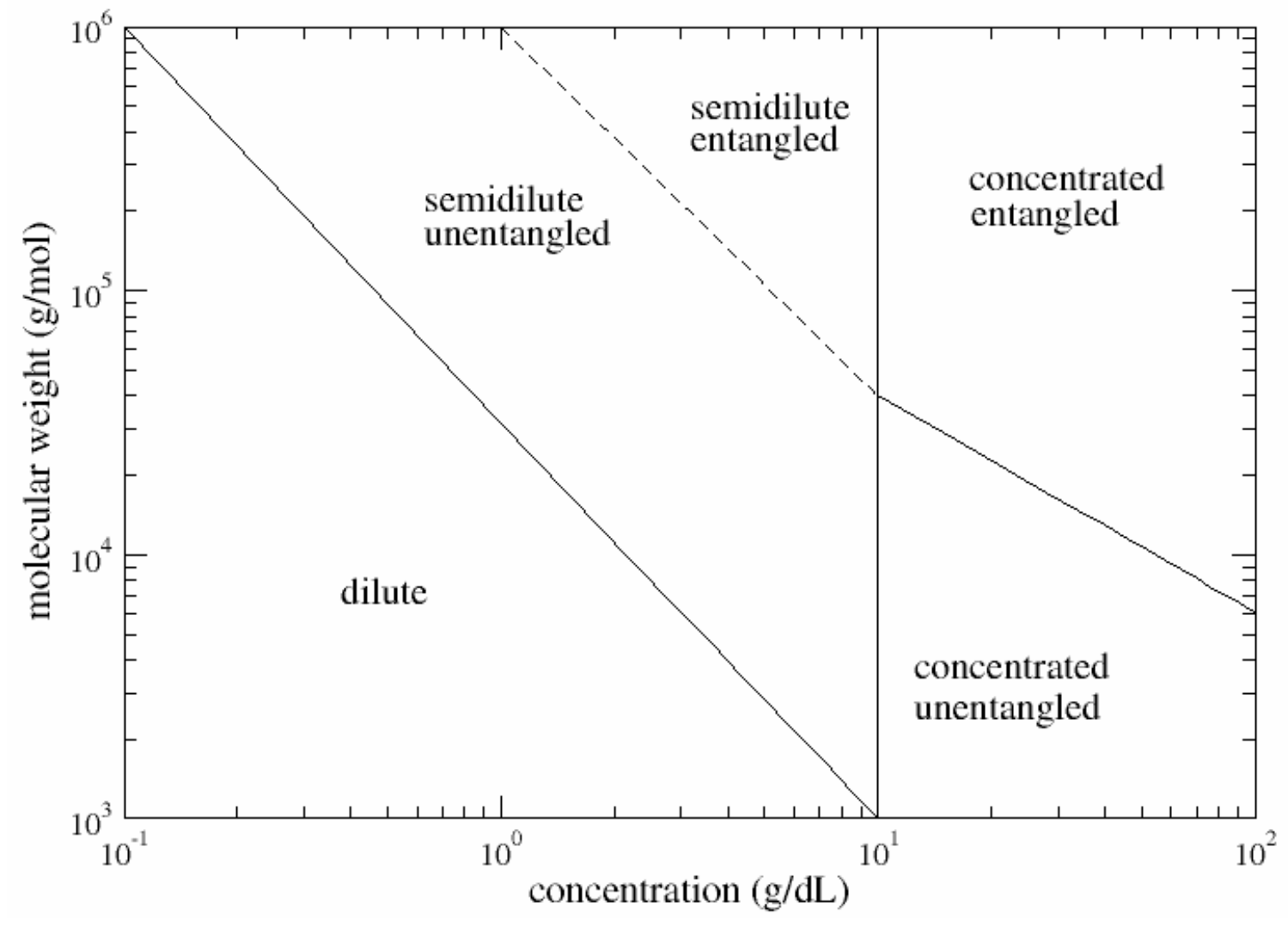

Figure 2.1. Classification of polymer solutions in terms of concentration and molecular weight ${ }^{1}$ 
The application of the Rouse model to semidilute unentangled solutions is reasonable because the hydrodynamic interactions and the excluded volume effects are screened out in these solutions. Raspaud et al. ${ }^{8}$ and Musti et al. ${ }^{10}$ published scaling plots of zero-shear viscosities versus concentration in the semidilute unentangled and entangled regimes for polystyrene, polyisoprene, and polybutadiene in good solvents and T2 phage DNA in buffered water, which is a good solvent for DNA, and showed that a common universal plot is obtained for all of them.

The theoretical basis of universal dynamic scaling in semidilute solution is the "blob" theory, ${ }^{5,11}$ in which a polymer is thought of as a chain of $N / g$ hydrodynamically independent blobs, where $N$ is the degree of polymerization and $g$ is the number of monomers in a blob, and the blob size is set by the condition that the polymer within the volume of a single blob belongs primarily to a single chain, while regions of space larger than a blob contain monomers from multiple polymer molecules. Thus, within a single blob, the polymer does not "know" that it is not in a dilute solution, and the dynamics are described by the dilute solution theory of Zimm, in which hydrodynamic interactions dominate. On scales larger than a blob, the polymer interacts hydrodynamically with other polymers, which screen out internal hydrodynamic interactions. If the chains are not so concentrated as to be entangled, the dynamics on scales larger than the blob size are therefore described by the Rouse theory. In fact, if one takes the "blob" to be a rescaled "monomer" of the chain, then on length scales larger than a blob, a semidilute solution can be described as a melt of chains of blobs. ${ }^{3-9}$ This rescaling of the effective "monomer" size implies that the concentration of the polymer and its length (or molecular weight) can be collapsed into a single scaling parameter, which is just the 
concentration $c$ divided by the critical polymer overlap concentration $c^{*} \equiv \frac{M}{N_{A} R_{g}{ }^{3}}$, where $M$ is the polymer molecular weight, $N_{A}$ is Avogadro's number, and $R_{g}$ is the radius of gyration of the polymer. Thus, if we draw a plot of $\eta_{s p}$ against $c / c^{*}$, where $\eta_{s p} \equiv\left(\eta-\eta_{s}\right) / \eta_{s}$ is the specific viscosity, the explicit effect of the polymer length disappears (i.e., it is submerged into the value of $c^{*}$; see Figure 4 in ref. 8). However, if the number of blobs in a chain surpasses the number needed for the chains to become entangled, a single scaling parameter is no longer sufficient, since the number of blobs needed to produce one entanglement depends on the microscopic chemical nature of the polymer. In the semidilute entangled regimes, we therefore must employ the entanglement concentration $c_{e}$ as another scaling parameter to obtain universal scaling behavior that is independent of chemical species.

In summary, the dynamics of the semidilute regime have been addressed by resorting to well-established theories for dilute solutions and entangled concentrated solutions or melts using scaling laws to normalize concentration and viscosity. If entanglements are absent, the specific viscosity plotted against concentration reduced by the overlap concentration will not be affected by the chemical nature of the chain, but will be weakly affected by the number of blobs per chain, $\eta_{\text {Rouse }}=\eta_{s}(N / g)$. As the concentration increases and reaches the point $c=c_{e}$, where the reduced degree of polymerization, $N / g$, equals the number of blobs per entanglement, $n_{e}$, the rescaled dynamics starts to be controlled by the chemical nature of each chain, which determines $n_{e}$ for entangled polymers. For entangled melts, we have $\eta / \eta_{\text {Rouse }} \approx\left(M / M_{e}\right)^{2.4}, 8,12,13$ since the zero-shear viscosity scales as $\eta \approx\left(M / M_{e}\right)^{3.4}$ in the entangled regime $e^{6,14}$ and the Rouse viscosity is 
proportional to $M$. In semidilute solutions, the number of entanglements per chain $\left(\frac{N / g}{n_{e}}\right)$ scales as $\frac{N / g}{n_{e}}=\left(\frac{c}{c_{e}}\right)^{1 /(3 v-1)}$. Since $\frac{N / g}{n_{e}}$ in semidilute solutions takes the place of $M / M_{e}$ for melts, we have for the former $\eta_{p} / \eta_{\text {Rouse }} \approx\left(c / c_{e}\right)^{2.4 /(3 v-1)}$.

\subsection{Experimental Data}

To test universal scaling thoroughly, we have collected literature data to compare to the scaling curve of Raspaud et al. ${ }^{8}$ The data include polystyrene in benzene, polystyrene in tricresyl phosphate, poly (ethylene oxide) in water, polyisoprene in cyclohexane, and polybutadiene in cyclohexane. In addition to these literature data, we shall here measure the zero-shear viscosity versus concentration for $\lambda$-DNA in Tris-EDTA buffer solution. In the following, we explain the experimental method for rheological measurement of these solutions.

\subsubsection{Sample Preparation}

We purchased $\lambda$-DNA from Invitrogen; as purchased the DNA is stocked in a storage buffer, $10 \mathrm{mM}$ Tris-HCl (pH 7.4), $5 \mathrm{mM} \mathrm{NaCl}$, and $0.1 \mathrm{mM}$ EDTA. Before we perform an experiment, we need to warm up the $\lambda$-DNA solution to $65{ }^{\circ} \mathrm{C}$ for 10 minutes and

quench it to bring it back to its linear form, since while stored at $4^{\circ} \mathrm{C}, \lambda$-DNA has tendency to make linear aggregates as well as circular structures due to the presence of cohesive overhangs. ${ }^{15}$ For this fixed-length DNA, the only parameter we can manipulate is the concentration. To control the concentration, we use a lyophilizer to dry out the 
sample without damaging the DNA itself, keeping the solution well below the freezing point to keep it from melting during the lyophilizing process, which can cause part of solution to spill out of the vial. Before the $\lambda$-DNA solution was put into a lyophilizer, we constructed a calibration curve to control the concentration of the $\lambda$-DNA. Assuming that the as-received concentration of the stock $\lambda$-DNA solution is correct, we made five individual $\lambda$-DNA solutions with different concentrations by dilution. UV absorbance at each concentration was measured with the Agilent 8453 UV spectrophotometer in the range of $0.3 \sim 1.0$ optical density where the relationship between concentration and absorbance follows the Beer-Lambert law, $A=\varepsilon b c$, where $A$ is the UV absorbance at the wavelength of $258 \mathrm{~nm}$ in arbitrary units, $\varepsilon$ the molar absorptivity in $\mathrm{Lg}^{-1} \mathrm{~cm}^{-1}, b$ the path length of the cuvette in $\mathrm{cm}$, and $c$ the concentration of the solution in $\mathrm{g} / \mathrm{L}$. We obtained a calibration curve with $\varepsilon=21.4 \pm 0.1 \mathrm{Lg}^{-1} \mathrm{~cm}^{-1}, b=1.0 \mathrm{~cm}$, and the concentration of the stock $\lambda$-DNA solution, $c=0.308 \mathrm{~g} / \mathrm{L}$. Based on this curve, we measured the concentration of each $\lambda$-DNA solution. This value of $\varepsilon$ agrees well with the widely accepted value of 20 $\mathrm{Lg}^{-1} \mathrm{~cm}^{-1}$ for double stranded DNA in water, indicating that the supplier's report $\lambda$-DNA concentration was correct. ${ }^{16,17}$

\subsubsection{Rheometry}

The range of concentrations that we can study is determined by the sensitivity of the rheometer. We used the Contraves Low Shear 30, since it is designed specially for lowviscosity fluids, and is sensitive enough to measure a zero-shear viscosity of around $2 \mathrm{cP}$ at a shear rate of 0.017 1/s. Figure 2.2 shows a schematic diagram of the Contraves rheometer. The measuring principle of this concentric-cylinder device is as follows. The 
inner bob is suspended by a torsion wire. A light beam reflected by a mirror attached to the bob is detected by a photocell and amplified while the cup is rotating. The amplified signal goes into the compensation system. The current required to zero this point is proportional to the reaction moment and therefore a function of the viscosity. The instrument measures the compensating torque needed to keep the torsion wire at its null position; hence at steady state there is no compliance of the torsion wire that needs correction. ${ }^{18}$

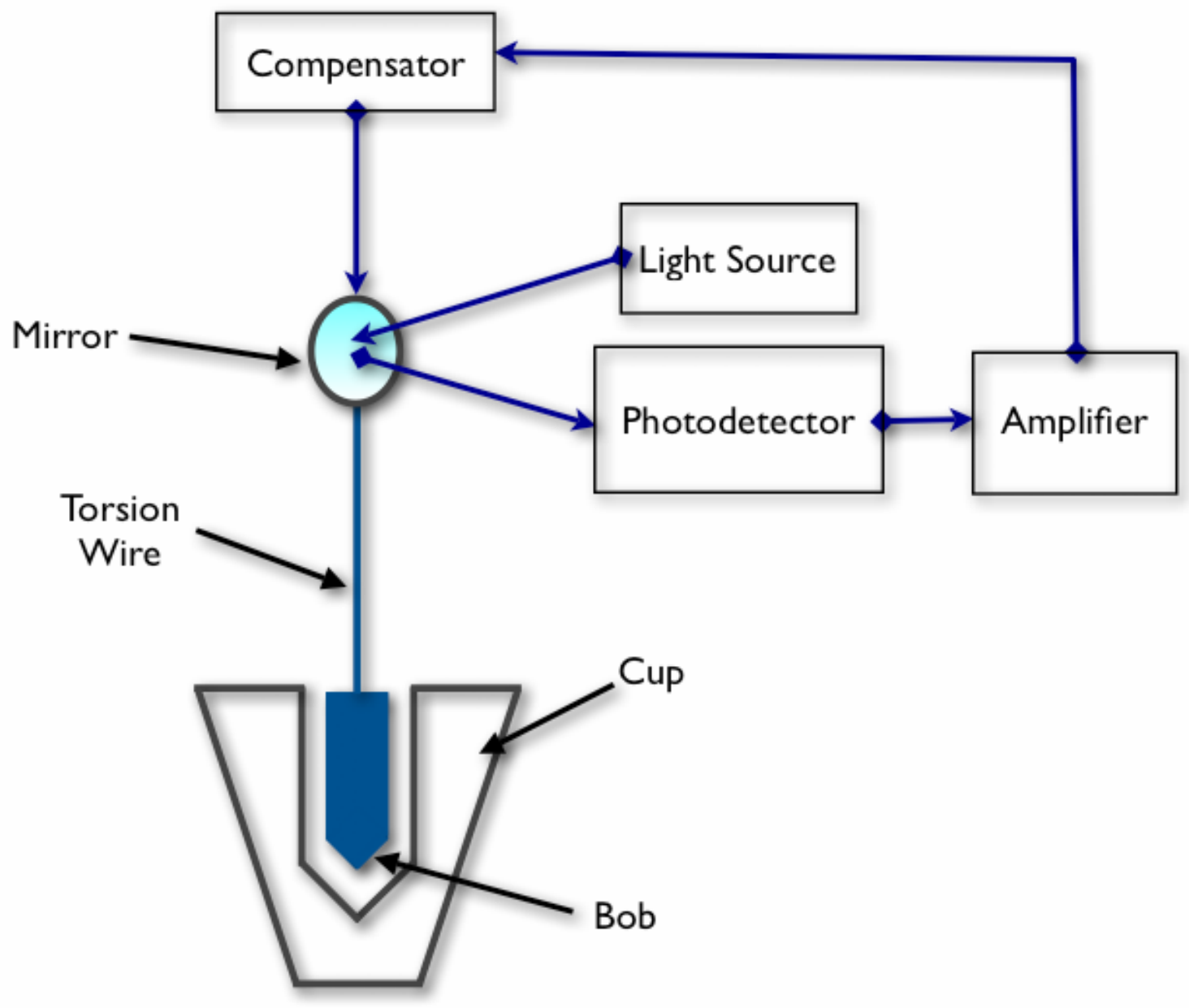

Figure 2.2. Schematic diagram of Contraves Low Shear 30 
To determine with confidence the zero shear viscosity of a sample, we need to obtain a constant viscosity over a range of at least one decade of shear rate. With bob radius of $R_{i}=5.5 \mathrm{~mm}$, cup inner radius of $R_{o}=6 \mathrm{~mm}$, bob length of $L=20 \mathrm{~mm}$, and bob underside cone angle $\alpha=20^{\circ}$ (see Figure 2.2), the lowest shear rate at which the Contraves Low Shear 30 can detect the signal is $0.0171 / \mathrm{s}$. Thus it is necessary that the crossover shear rate at which shear thinning transitions to the zero-shear plateau be greater than $0.17 \mathrm{1} / \mathrm{s}$. From this, we can estimate that for $\lambda$-phage DNA, the highest concentration we can measure is about $0.7 \mathrm{~g} / \mathrm{L}$ (see Figure 2.3). One disadvantage of the rheometer is that it takes at least 30 minutes to run one experiment and the filling volume is small $(1.5 \mathrm{ml})$, so that blocking water from evaporating during rheometry is an important task.

To minimize evaporation, we put a kimwipe tissue soaked with buffer solvent in the cylinder-shape container surrounding the cup-and-bob system, and checked how much evaporation occurred both by repeating the measurement at the first shear rate at which the viscosity was obtained after all other data were measured on that sample, and by remeasuring the concentration with UV spectrophotometry after unloading the sample. The results showed that our method of blocking evaporation is reliable, with changes in concentration less than $5 \%$. All rheometry experiments with $\lambda$-DNA were carried out at $25.0 \pm 0.3^{\circ} \mathrm{C}$. 


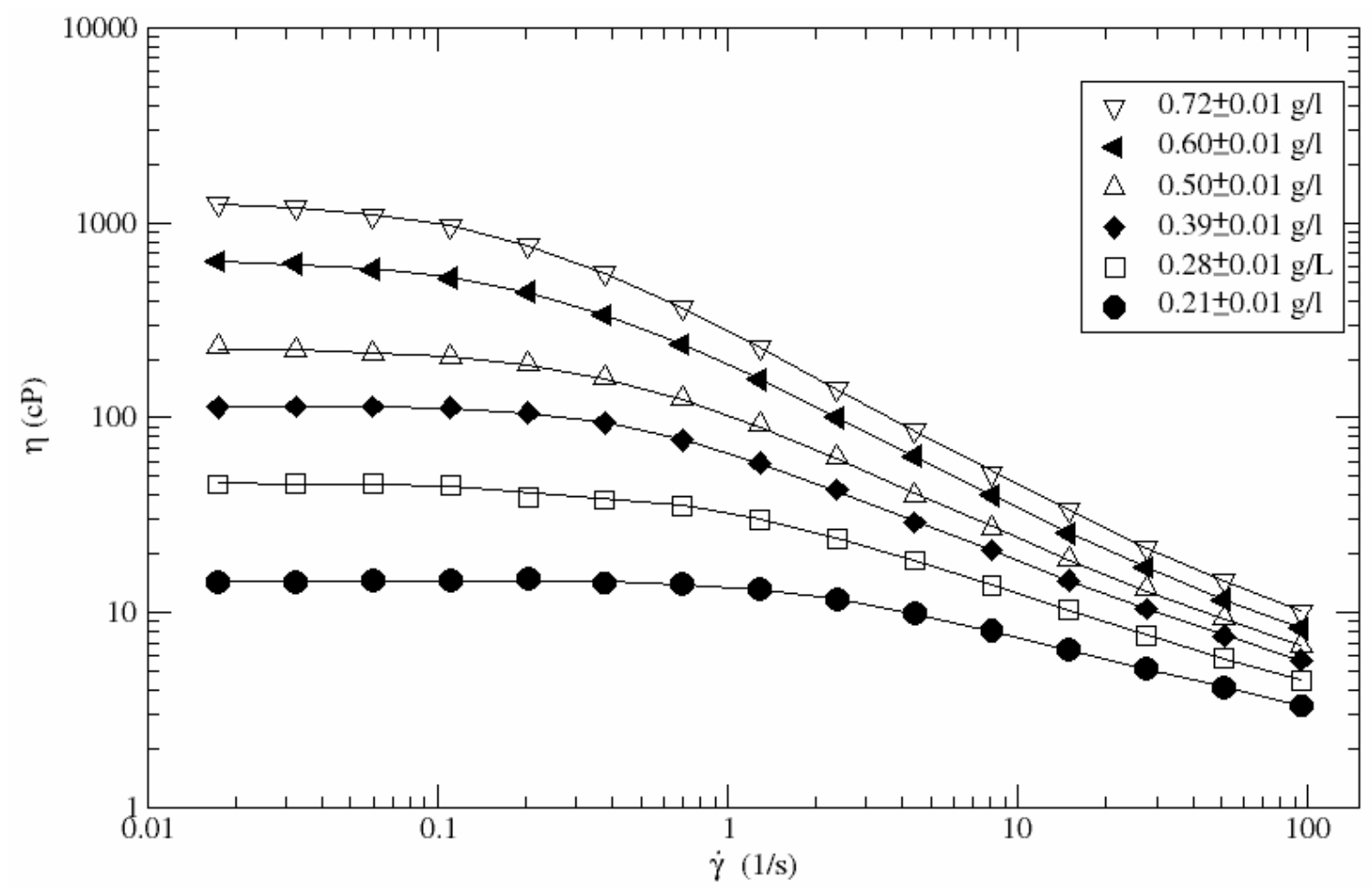

Figure 2.3. Dependence of shear viscosity on shear rate for $\lambda$-DNA solutions for several concentrations at $25.0 \pm 0.3^{\circ} \mathrm{C}$. The lines are the fits of the Carreau-Yasuda model with parameters of the fit given in Table 2.1.

\subsection{Results and Discussion}

\subsubsection{Determining the Zero-shear Viscosity}

\section{Carreau-Yasuda model}

Our measured shear viscosities for $\lambda$-DNA solutions are shown in Figure 2.3, along with fits of the Carreau-Yasuda model, which is

$$
\frac{\eta-\eta_{\infty}}{\eta_{0}-\eta_{\infty}}=\left[1+(\lambda \dot{\gamma})^{a}\right]^{(n-1) / a}
$$

where $\eta_{0}$ is the zero-shear-rate viscosity, $\eta_{\infty}$ is the infinite-shear-rate viscosity, $\lambda$ is a time constant where $1 / \lambda$ is the critical shear rate at which viscosity begins to decrease, $(n-1)$ is the power law exponent (since it describes the slope of $\left(\eta-\eta_{\infty}\right) /\left(\eta_{0}-\eta_{\infty}\right)$ in the 
power-law region) and $a$ is a dimensionless parameter that describes the width of transition between the zero-shear-rate region and the power-law region. We have chosen this model because it is quite flexible in fitting the non-Newtonian behavior of $\eta(\hat{\gamma})$ over a wide range of shear rates.

The fits to the Carreau-Yasuda equation were obtained using simulated annealing, which is a nonlinear iterative random search procedure with adaptive moves along the coordinate directions. It permits uphill moves using a Metropolis algorithm, and is thus able to avoid becoming trapped in local minima and can find the global best fit. ${ }^{19}$ We used the fits to the Carreau-Yasuda model to determine the zero-shear viscosity, although a reasonable value can also be obtained from the viscosity at the lowest measured shear rate; see table 2.1. There exists a clear trend in the dependence of all five parameters of the Carreau-Yasuda fits on concentration. However, we found for one concentration, $c=0.28 \mathrm{~g} / \mathrm{L}$, that there were large deviations from this trend, unless we dropped the data points at shear rates of 0.20 and $0.38 \mathrm{1} / \mathrm{s}$ where the data appear to show a small discontinuity. Once these two data points were dropped, the parameters for $c=0.28 \mathrm{~g} / \mathrm{L}$ followed the monotonic trends observed for the other concentrations, except for the parameter $a$, as shown in Table 2.1. These results indicate that the five-parameter Carreau-Yasuda model has as many parameters as can be meaningfully fit to our data. We also fit our data with a three-parameter model, the Cross model, but this model did not give good fits to the zero-shear viscosity and so we pursued it no further here. 
Table 2.1. The parameter values of the Carreau-Yasuda curvefit to shear thinning curve of $\lambda$-DNA solution for various concentrations. For the concentration $\mathrm{c}=0.28 \mathrm{~g} / \mathrm{L}$, the data in the shear rate range $0.20-0.38 \mathrm{~s}^{-1}$ were omitted when fitting the parameters, since the parameters were highly sensitive to slight noise in the these few data point; see discussion in text.

\begin{tabular}{cccccc}
\hline$c(\mathrm{~g} / \mathrm{L})$ & $\eta_{0}(\mathrm{cP})$ & $\eta_{\infty}(\mathrm{cP})$ & $\lambda(\mathrm{s})$ & $a$ & $N$ \\
\hline 0.21 & 15 & 0.47 & 0.55 & 1.7 & 0.60 \\
0.28 & 47 & 1.3 & 1.1 & 1.1 & 0.42 \\
0.39 & 110 & 1.6 & 2.1 & 1.6 & 0.37 \\
0.50 & 230 & 2.8 & 2.3 & 1.3 & 0.26 \\
0.60 & 650 & 3.3 & 4.1 & 1.2 & 0.19 \\
0.72 & 1300 & 4.0 & 5.7 & 1.3 & 0.15 \\
\hline
\end{tabular}

\subsubsection{The Zero-shear Viscosities of $\lambda$-DNA Solutions and the Universal Dependence on Concentration}

In order to reveal the effect of chain length on rheological behavior, first we need to define the chain dimensions of each polymer. There are at least four dilute solution properties associated with the size of the polymer chain. These include the radius of gyration, $R_{g}$, the second virial coefficient, $A_{2}$, the intrinsic viscosity, $[\eta]_{0}$, and the diffusion coefficient $D_{0}$. In dilute polymer solutions, for scaling purposes each polymer coil is thought of as a hard sphere whose interior is shielded from the flow due to hydrodynamic interactions. ${ }^{20}$ Thus, we can express the contribution of suspended polymer molecules on viscosity by using the Einstein formula:

$$
\eta_{0}-\eta_{s}=2.5 \eta_{s} \phi
$$

where $\eta_{0}$ is the zero shear viscosity, $\eta_{s}$ the solvent viscosity and $\phi$ the equivalent "hard sphere” volume fraction of polymer chains in solution. If we divide both sides of Eq. (2) by the mass concentration, $c$, then we have the following equation with $\phi=v V_{p e r}$ and $c=v M_{w} / N_{A}$ where $V_{p e r}$ is the "pervaded volume" or volume occupied by a hard sphere 
representation of the polymer molecule, $M_{w}$ is the weight-averaged molecular weight, $N_{A}$ Avogadro's number, and $v$ the number density of coils:

$$
[\eta]_{0}=2.5 \frac{N_{A} V_{p e r}}{M_{w}}
$$

To define a viscometric radius $R_{v}$ of the polymer, we take

$$
V_{p e r}=\frac{4 \pi}{3} R_{v}^{3}
$$

Then, from Eqs. (3) and (4), we obtain the viscometric radius of the polymer coil as:

$$
R_{v}=\left(\frac{3[\eta]_{0} M_{w}}{10 \pi N_{A}}\right)^{1 / 3}
$$

Once we know the ratio of $R_{v}$ to $R_{g}$, we can extract $[\eta]_{0}$ from $R_{g}$, or $R_{g}$ from $[\eta]_{0}$. The average experimental ratio of $R_{v}$ to $R_{g}$ is 0.76 for linear polymers in good solvents (see Table 6.1 in ref. 20). This value can also be obtained by combining Eq. (5) and the Flory-Fox parameter ${ }^{21} \Phi=\frac{[\eta]_{0} M_{w}}{6^{3 / 2} R_{g}^{3}}=1.9 \times 10^{23}$ where $\Phi$ has been determined by Monte Carlo simulation for bead-spring chains with excluded volume interactions, ${ }^{22}$ while the ratio of $R_{v}$ to $R_{g}$ derived by renormalization group (RG) theory for long chains in good dilute solvents is $0.73 .^{20,23}$ Using 0.76 for the ratio of $R_{v}$ to $R_{g}$, we have the following relationship.

$$
\frac{M_{w}}{N_{A} R_{g}^{3}}=\frac{4.6}{[\eta]_{0}}
$$

The effective radius of a polymer coil can also be defined using the second virial coefficient $A_{2}$. For a hard sphere, $A_{2}=\frac{4 N_{A} V_{p e r}}{M^{2}}$, where $V_{p e r}=\frac{4 \pi R_{t}^{3}}{3}$, and $R_{t}$ is the 
thermodynamic radius of an equivalent hard sphere model of the polymer. ${ }^{22}$ Hence we have

$$
R_{t}=\left(\frac{3 A_{2} M_{w}^{2}}{16 \pi N_{A}}\right)^{1 / 3}
$$

In the same way as for $R_{v}$, we can use the universal ratio of $R_{t}$ to $R_{g}$ obtained for goodsolvent conditions and the definition

$$
c^{*} \equiv \frac{M_{w}}{N_{A} R_{g}^{3}}
$$

to obtain $c^{*} M_{w} A_{2}$ for a given polymer species. For various synthetic polymers in various good solvents, ${ }^{22}$ we get an average value of 0.68 for $R_{t} / R_{g}$. Then,

$$
C^{*} \equiv \frac{M_{w}}{N_{A} R_{g}^{3}}=\frac{5.3}{M_{w} A_{2}}
$$

To organize literature data, we need some parameters that were not measured experimentally, and therefore we use the above universal ratios to estimate them. For example, the radius of gyration of $\lambda$-phage DNA has been measured to be $500 \mathrm{~nm}^{24}$ and the molecular weight is $3.15 \times 10^{7} \mathrm{~g} / \mathrm{mol}$, giving us $c^{*}$, but the second virial coefficient was not reported. Hence, we use Eq. (9) to get $M_{w} A_{2}$ with $C^{*}$ from Eq. (8).

In normalizing the viscosity and the concentration of polymer solutions, we have several choices. To normalize the concentration, we can use a coil-coil "overlap" concentration, which has been defined variously as

$$
c^{*} \equiv \frac{M_{w}}{N_{A} R_{g}^{3}} \text { (see Eq. (8)) or } c_{\eta}^{*} \equiv \frac{1}{[\eta]_{0}} \text { or } c_{A}^{*} \equiv \frac{1}{M_{w} A_{2}}
$$

Raspaud et al. ${ }^{8}$ normalized the polymer viscosity as $\eta_{p} / \eta_{\text {Rouse }}$ with $\eta_{\text {Rouse }}=\eta_{s}\left(c / c_{\eta}^{*}\right)^{1 /(3 v-1)}$ and normalized concentration as $c / c_{e}$ with $c_{e}=c_{A}^{*} n_{e}^{3 v-1}$. Note that they chose the overlap 
concentration $c_{\eta}^{*} \equiv 1 /[\eta]_{0}$ for $\eta_{\text {Rouse }}$, but to compute $c_{e}, c_{A}^{*} \equiv 1 /\left(M A_{2}\right)$ was used, even though consistency would seem to require using the same definition of crossover concentration to rescale both the viscosity and the concentration. They used different definitions of the overlap concentration for these two rescalings because they thereby attained a better collapse of data for multiple polymers onto the same scaling curve. We will therefore follow this procedure as well.

From the scaling curves of many polymer-solvent systems with excluded volume exponent lying above 0.5 (where $v=0.5$ corresponds to a theta solvent) up to 0.588 (corresponding to good solvent conditions), we can draw the conclusion that the linear rheological properties of any polymer-good solvent system can be predicted by using a plot of normalized viscosity vs. normalized concentration for a fixed solvent quality, e.g., for "good" solvents. However, since the "good" solvent limit is often not completely achieved and the solvent quality varies somewhat with polymer-solvent pair, the plots can be expected to show some scatter, even though we have attempted to account for varying solvent quality by using literature values of parameters, such as $R_{g}$ and $A_{2}$ or computing these from literature values of the excluded volume exponent $v$ (which ranged from 0.530 to 0.585 ); see Table 2.2. Nevertheless, there is a clear power-law scaling of $\eta_{p} / \eta_{\text {Rouse }}=(43 \pm 2) \times\left(c / c_{e}\right)^{3.12 \pm 0.05}$ for $c / c_{e}>0.50$ (see Figure 2.4). The scaling exponent is robust. If we change the lower limit of concentrations included in the fit, the value of the exponent remains constant at around 3.12 to within \pm 0.01 for any lower limit from $c / c_{e}=0.5$ up to $c / c_{e}=1.0$. This result is roughly consistent with the expected scaling $\eta_{p} / \eta_{\text {Rouse }} \propto\left(c / c_{e}\right)^{2.4 /(3 v-1)}=\left(c / c_{e}\right)^{3.1}$ for entangled semidilute solutions 
Table 2.2. Scaling parameters for various polymer-solvent pairs at various temperatures

\begin{tabular}{|c|c|c|c|c|c|c|c|c|c|c|c|}
\hline polymer & solvent & $\begin{array}{l}\text { temp } \\
\left({ }^{\circ} \mathrm{C}\right)\end{array}$ & ref. $^{\text {a }}$ & $v^{\mathrm{b}}$ & $\begin{array}{l}M_{w} \\
\left(10^{6} \mathrm{~g} / \mathrm{mol}\right)\end{array}$ & $\begin{array}{l}R_{g} \\
(\mathrm{~nm})\end{array}$ & $\begin{array}{l}c^{* \mathrm{c}} \\
(\mathrm{g} / \mathrm{L})\end{array}$ & $\begin{array}{l}{[\eta]_{0}} \\
(\mathrm{~L} / \mathrm{g})\end{array}$ & $\begin{array}{l}M_{w} A_{2} \\
(\mathrm{~L} / \mathrm{g})\end{array}$ & $n_{e}{ }^{\mathrm{d}}$ & $\begin{array}{l}c_{e}{ }^{\mathrm{e}} \\
(\mathrm{g} / \mathrm{L})\end{array}$ \\
\hline T2 phage DNA & $\begin{array}{l}\text { buffered } \\
\text { water }\end{array}$ & 30 & 1 & $0.577^{\mathrm{f}}$ & $105^{\mathrm{g}}$ & $1000^{\mathrm{h}}$ & 0.174 & $30^{\mathrm{i}}$ & $30^{\mathrm{j}}$ & $16^{\mathrm{k}}$ & 0.25 \\
\hline$\lambda$-phage DNA & $\begin{array}{l}\text { buffered } \\
\text { water }\end{array}$ & 25 & & $0.577^{\mathrm{f}}$ & 31.5 & $500^{1}$ & 0.419 & $13^{\mathrm{m}}$ & $13^{\mathrm{i}}$ & $16^{\mathrm{k}}$ & $0.60^{\mathrm{n}}$ \\
\hline $\begin{array}{l}\text { poly (ethylene } \\
\text { oxide) }\end{array}$ & water & 25 & 2 & $0.583^{\circ}$ & 0.333 & $36^{\circ}$ & 12.2 & $0.376^{\mathrm{P}}$ & $0.482^{\mathrm{q}}$ & $100^{r}$ & 65.3 \\
\hline polystyrene & benzene & 30 & 3 & $0.583^{\mathrm{S}}$ & $\begin{array}{l}4.00 \\
1.24 \\
\end{array}$ & $\begin{array}{r}110 \\
54 \\
\end{array}$ & $\begin{array}{r}5.0 \\
12.9 \\
\end{array}$ & $\begin{array}{l}0.7^{\mathrm{t}} \\
0.3^{\mathrm{t}}\end{array}$ & $\begin{array}{l}1.27 \\
0.52\end{array}$ & 185 & $\begin{array}{l}39.3 \\
96.0 \\
\end{array}$ \\
\hline polystyrene & $\begin{array}{l}\text { tricresyl } \\
\text { phosphate }\end{array}$ & 25 & 4 & $0.530^{\mathrm{u}}$ & 1.80 & $53^{v}$ & 19.7 & $0.206^{\mathrm{u}}$ & $0.256^{j}$ & 185 & 81.4 \\
\hline polystyrene & benzene & 35 & 5 & $0.583^{\mathrm{x}}$ & $\begin{array}{l}0.17 \\
0.42 \\
1.26 \\
2.89 \\
3.84 \\
6.77 \\
20.6 \\
\end{array}$ & $\begin{array}{r}18.8^{\mathrm{y}} \\
32.2^{\mathrm{y}} \\
61.8^{\mathrm{y}} \\
101.3^{\mathrm{y}} \\
120.0^{\mathrm{y}} \\
168.1^{\mathrm{y}} \\
325.9^{\mathrm{y}}\end{array}$ & $\begin{array}{r}42.50 \\
20.91 \\
8.86 \\
4.62 \\
3.70 \\
2.37 \\
0.99 \\
\end{array}$ & $\begin{array}{l}0.066^{\mathrm{x}} \\
0.129^{\mathrm{x}} \\
0.293^{\mathrm{x}} \\
0.547^{\mathrm{x}} \\
0.677^{\mathrm{x}} \\
1.035^{\mathrm{x}} \\
2.385^{\mathrm{x}}\end{array}$ & $\begin{array}{l}0.125 \\
0.253 \\
0.598 \\
1.148 \\
1.434 \\
2.239 \\
5.362 \\
\end{array}$ & 185 & $\begin{array}{r}402.3 \\
197.9 \\
83.9 \\
43.7 \\
35.0 \\
22.4 \\
9.4 \\
\end{array}$ \\
\hline polyisoprene & cyclohexane & 30 & 3 & $0.580^{\mathrm{S}}$ & $\begin{array}{l}0.94 \\
0.31 \\
\end{array}$ & $\begin{array}{l}54 \\
28 \\
\end{array}$ & $\begin{array}{r}9.9 \\
23.5 \\
\end{array}$ & $\begin{array}{l}0.470 \\
0.210 \\
\end{array}$ & $\begin{array}{l}0.650 \\
0.300 \\
\end{array}$ & 112 & $\begin{array}{r}51 \\
109 \\
\end{array}$ \\
\hline polybutadiene & cyclohexane & 30 & 3 & $0.583^{\mathrm{s}}$ & 0.96 & 53 & 10.7 & 0.6 & 0.610 & 38 & 25 \\
\hline
\end{tabular}

a $1=$ ref. 10; 2=ref. 25; 3=ref. 8; 4=ref. 26; and 5=ref. 27

${ }^{\mathrm{b}}$ excluded volume exponent, $v=(a+1) / 3$ with $a$ the Mark-Houwink exponent

c overlap concentration, $c^{*}=M_{w} /\left(N_{A} R_{g}^{3}\right)$ with $N_{A}$ Avogadro's number 
d number of monomers between entanglements, $n_{e}=M_{e} / m_{0}$ with $M_{e}$ the entanglement molecular weight, and $m_{0}$ the monomer molecular weight

e entanglement concentration, $c_{e}=n_{e}^{(3 v-1)} / M_{w} A_{2}$

f ref. 28-31

g ref. 32

h determined by scaling using $\lambda$-DNA with $R_{g}=500 \mathrm{~nm}^{24}$ and $R_{g} \sim M_{w}^{v}$ with $v=0.577^{29}$

i ref. 33

${ }^{j}$ calculated from Eq. (9), $c^{*}=\frac{5.3}{M_{w} A_{2}}$ and using $c^{*}=\frac{M_{w}}{N_{A} R_{g}^{3}}{ }^{20}$

k calculated from $n_{e}=\left(c_{e} \times M_{w} A_{2}\right)^{1 /(3 v-1)}$ with $c_{e}=0.25 \mathrm{~g} / \mathrm{l}^{8,10}$

${ }^{\mathrm{l}}$ ref. 24

${ }^{\mathrm{m}}$ For identical polymer species at the same or similar temperature, the value of $[\eta]_{0} c^{*}$ is the same irrespective of molecular weight within $10 \%$ error. ${ }^{8}$ Hence, we can use $[\eta]_{0}=5.2 / c^{*}$ from T2 phage DNA data

G $\quad{ }^{\mathrm{n}}$ determined from $c_{e}=n_{e}^{3 v-1} /\left(M_{w} A_{2}\right)$ with $n_{e}=16^{\mathrm{k}}, v=0.577^{\mathrm{f}}$, and $M_{w} A_{2}=13^{\mathrm{j}}$

${ }^{\circ} R_{g}=0.0215 M_{w}^{0.583 \pm 0.031} \mathrm{~nm}^{34}$

${ }^{\mathrm{P}} R_{v} / R_{g}=0.84$ for linear polymers in good solvents ${ }^{20,23}$

${ }^{\mathrm{q}} A_{2}=1.84 \times 10^{-2} \mathrm{M}_{w}^{-0.20 \pm 0.06} \mathrm{~mL} \cdot \mathrm{mol} / \mathrm{g}^{234}$

${ }^{\mathrm{r}} M_{e}=4400 \mathrm{~g} / \mathrm{mol}^{35}$

${ }^{s}$ ref. 36

${ }^{\mathrm{t}}$ ref. 8

u ref. 37; The excluded volume exponent is insignificantly higher than 0.5 . See the text.

${ }^{v} R_{v} / R_{g}=0.74$ for linear polystyrene solutions over the range of $10^{5} \leq M \leq 10^{6}$ (see Table 6.1 in ref. 20)

${ }^{\mathrm{w}} R_{\mathrm{g}}=0.012 M_{\mathrm{w}}^{0.585} \mathrm{~nm}^{38}$

х $[\eta]_{0}=7.8 \times 10^{-6} M_{v}^{0.75}(\mathrm{~L} / \mathrm{g})^{39}$

${ }^{\mathrm{y}} R_{g}=1.45 \times 10^{-2} M_{w}^{0.595}(\mathrm{~nm})^{40}$ 
in good solvent with $v=0.588$. Note that even though tricresyl phosphate is only marginally better than theta solvent, its data point remains on the single universal curve. However, further data for solutions with solvents only marginally better than theta will be required to establish that such solutions follow the same good-solvent scaling.

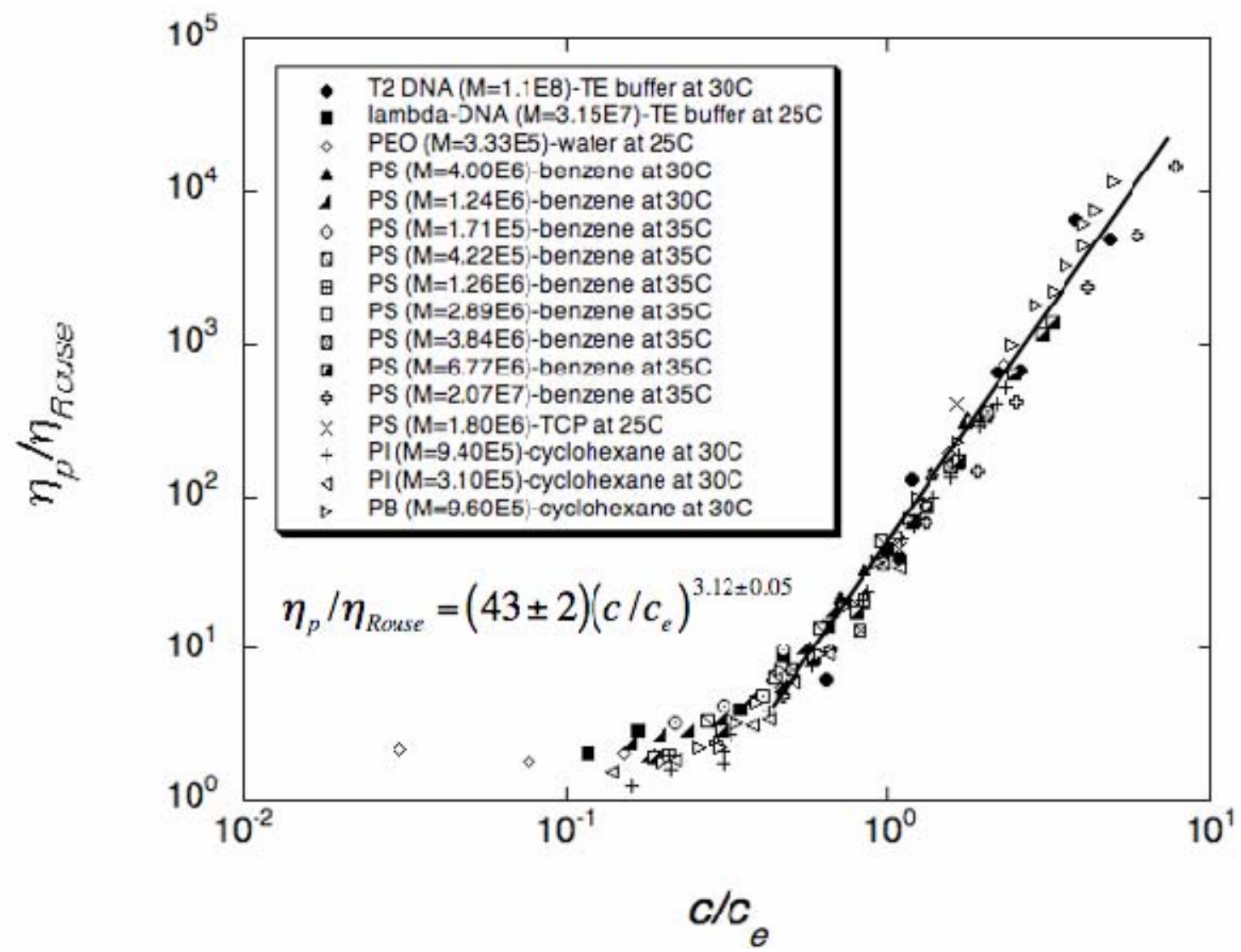

Figure 2.4. Master scaling curve of normalized zero shear viscosities of polymer solutions against normalized concentrations. $M$ stands for the weight-averaged molecular weight, $\mathrm{C}$ for degrees Celsius, TE for Tris-EDTA, PEO for poly (ethylene oxide), PS for polystyrene, TCP for tricresyl phosphate, PI for polyisoprene, and PB for polybutadiene. $\eta_{p}$ is the polymer contribution to zero shear viscosity, $\eta_{\text {Rouse }}$ is the hypothetical Rouse viscosity, and $c_{e}$ is the entanglement concentration. See text for detail.

Raspaud et al. ${ }^{8}$ used the same excluded volume exponent, $v=0.588$ for all three polymer species. But, when we include more polymer-solvent pairs, and use the appropriate excluded volume exponent corresponding to each pair (including the solvent- 
polymer pairs considered by Raspaud et al. ${ }^{8}$ ) we find the scaling law mentioned above, $\eta_{p} / \eta_{\text {Rouse }}=(43 \pm 2) \times\left(c / c_{e}\right)^{3.12 \pm 0.05}$. From this scaling law, the zero-shear viscosity for an arbitrary polymer-solvent pair at a given concentration can be estimated, once the values of the solvent viscosity, $\eta_{s}$, the intrinsic viscosity, $[\eta]_{0}$, the excluded volume exponent, $v$, the number of blobs per entanglement, $n_{e}=M_{e} / M_{0}$ with $M_{e}$ the entanglement molecular weight and $M_{0}$ the monomer molecular weight, and the second virial coefficient, $A_{2}$ are given. If the intrinsic viscosity or the second virial coefficient or both are unavailable from experiments but the radius of gyration, $R_{g}$ is known, we can extract these values from Eqs. (6) and (9) respectively.

\subsection{Conclusion}

We have measured the viscosities of aqueous $\lambda$-phage DNA solutions at $25.0 \pm 0.3^{\circ} \mathrm{C}$ with concentrations between the overlap concentration $c^{*}$ and the entanglement concentration $c_{e}$ as functions of shear rate with the Contraves rheometer. In addition, we collected sets of zero-shear viscosity versus concentration data for various polymersolvent- systems described in the literature. After normalizing the viscosity with the Rouse viscosity and the concentration using the entanglement concentration, all data lie approximately on a single universal curve with a power law scaling, $\eta_{p} / \eta_{\text {Rouse }}=(43 \pm 2) \times\left(c / c_{e}\right)^{3.12 \pm 0.05}$ for $c / c_{e}>0.5$. Moreover, the values of $\eta_{\text {Rouse }}$ and $c_{e}$ can be obtained from the polymer radius of gyration, the excluded volume exponent $v$, the entanglement molecular weight in the melt $M_{e}$, and the solvent viscosity $\eta_{s}$ so that a 
prior estimates of zero-shear viscosities for entangled polymer solutions can be made for any polymer in a good solvent.

We note the content of this chapter is reproduced with permission from Journal of Rheology (copyright 2005, 2007 American Institute of Physics). 


\section{References}

1. Graessley, W. W. Polymer, 1980, 21, 258-262.

2. Daoud, M.; Cotton, J. P.;Farnoux, B.; Jannink, G.; Sarma, G.; Benoit, H.; Duplessix, R.; Picot, C.; de Gennes, P. -G. Macromolecules, 1975, 8, 804-818.

3. de Gennes, P. -G. Macromolecules, 19776a, 9, 587-593.

4. de Gennes, P. -G. Macromolecules, 1976b, 9, 594-598.

5. de Gennes, P. -G., Scaling Concepts in Polymer Physics; Cornell University Press: Ithaca, NY, 1979.

6. Doi, M.; Edwards, S. F. The Theory of Polymer Dynamics; Oxford Science, Oxford, U. K., 1986.

7. Colby, R. H.; Rubinstein, M.; Daoud, M. J.Phys. II. France, 1994, 4, 1299-1310.

8. Raspaud, E.; Lairez, D.; Adam, M. Macromolecules, 1995, 28, 927-933 (1995).

9. Rubinstein, M.; Colby, R. H. Polymer Physics; Oxford University Press, New York, 2003.

10. Musti, R.; Sikorav, J. L.; Lairez, D.; Jannink, G.; Adam, M. C. R. Acad. Sci. Paris, t. Serie II b, 1995, 320, 599-605.

11. Colby, R. H.; Rubinstein, M. Macromolecules, 1990, 23, 2753-2757.

12. Menezes, E. V.; Graessley, W. W. J. Polym. Sci. Part B-Polym. Phys. Ed., 1982, 20, 1817-1833.

13. Osaki, K.; Inoue, T.; Uematsu, T.; Yamashita, Y. J. Polym. Sci. Part B-Polym. Phys. Ed. 2001, 39, 1704-1712.

14. Berry, G. C.; Fox, T. G. Adv. in Polym. Sci. 1968, 5, 261-357.

15. Sanger, F.; Coulson, A. R.; Hong, G. F.; Hill, D. F.; Petersen, G. B. J. Mol. Bio. 1982, 162, 729-773.

16. Gallagher, S. R. Current Protocols In Molecular Biology, Vol. 2.: John Wiley \& Sons, Inc., New York, 1995. 
17. Sambrook, J.; Fritsch, E. F.; Maniatis, T. Molecular Cloning: A Laboratory Manual, $2^{\text {nd }}$ ed.: CSH Laboratory Press, Cold Spring Harbor, New York, 2000.

18. Colby, R. H., personal communication, 2004.

19. Corana, A.; Marchesi, M.; Martini, C.; Ridella, S. ACM Transactions on Mathematical Software, 1987, 13, 262-280.

20. Graessley, W. W. Polymeric Liquids and Networks: Structure and Properties: Garland Science, 2004.

21. Flory, P. J.; Fox, T. G. J. Am. Chem. Soc., 1951, 73, 1904-1908.

22. Bernal, J. M. G.; Tirado, M. M.; Freire, J. J.; de la Torre, J. G. Macromoleulces, 1991, 24, 593-598.

23. Fujita, H., Polymer Solutions: Elsevier, Amsterdam, 1990.

24. Verma, R.; Crocker, J. C.; Lubensky, T.C.; Yodh, A. G. Phys. Rev. Lett., 1998, 81, 4004-4007.

25. van Zanten, J. H.; Amin, S.; Abdala, A. A. Macromolecules, 2004, 37, 3874-3880.

26. Graessley, W. W.; Park, W. S.; Crawley, R. L. Rheol. Acta., 1977, 16, 291-301.

27. Adam, M.; Delsanti, M. J. Physics. France, 1983, 44, 1185-1193.

28. Dondos, A. Polymer, 2000, 41, 4607-4616.

29. Dondos, A. European Polymer Journal, 2002, 38, 1311-1317.

30. Rice, S. A.; Doty, P. J. Am. Chem. Soc., 1957, 79, 3937-3947.

31. Doty, P.; Marmur, J.; Eigner, J.; Schildkraut, C. Proc. Nat'l. Acad. Sci., 1960, 46, 461-476.

32. Larson, R. G. J. Rheol., 2005, 49, 1-70.

33. Chapman, R. E.; Klotz, L.C.; Thompson, D. S.; Zimm, B. H. Macromolecules, 1969, 2, 637-643.

34. Devanand, K.; Selser, J. C. Macromolecules, 1991, 24, 5943-5947.

35. Dontula, P.; Macosko, C. W.; Scriven, L.E. AIChE J., 1998, 44, 1247-1255. 
36. Brandrup, J.; Immergut, E. H.; Grulke, E. A. Polymer Handbook, $4^{\text {th }}$ ed.:Wiley, New York, 1999.

37. Solomon, M. J.; Muller, S. J. J. Polym. Sci.: Part B: Polym. Phys., 1996, 34, 181-192.

38. Pedersen, J. S.; Schurtenberger, P. J. Polym. Sci.: Part B: Polym. Phys., 2004, 42, 3081-3094.

39. Einaga, Y.; Miyaki, Y.; Fujita, H. J. Polym. Sci., Polym. Phys. Ed., 1979, 17, 21032109.

40. Decker, D. Thesis, Strasbourg, 1968. 


\title{
Chapter 3
}

\section{Universal Scaling of Linear and Nonlinear Rheological Properties of Semidilute and Concentrated Polymer Solutions}

\begin{abstract}
While it has been previously demonstrated that the concentration dependence of the zero-shear viscosity of most semidilute polymer solutions in good solvents follows a universal scaling law derived from the de Gennes "blob" concept, ${ }^{1-3}$ we here examine more thoroughly the validity of the blob model in predicting both linear and nonlinear rheological properties of semidilute polymer solutions. To do so, we perform both oscillatory and steady shear rheometry on polystyrene (PS) solutions in tricresyl phosphate (TCP) with three nearly monodisperse molecular weights at six values of the reduced concentration $c / c_{e}$, where $c_{e}$ is the entanglement concentration. Bidisperse polystyrene solutions with molecular weights in a fixed ratio of long to short chain length at the same $c / c_{e}$ are also explored as a first step to confirm the validity of the universal "blob" model scaling for polydisperse polymer solutions. At the same $c / c_{e}$ below a critical value of around 2.0 for our PS/TCP solutions, linear and nonlinear rheological functions are successfully superimposed after the modulus and the frequency (or shear rate) of each solution are respectively normalized with the concentration-dependent plateau modulus $G_{N}^{0}$, and the equilibration time $\tau_{e, \text { scaling }}$ obtained from the de Gennes scaling relationships using the literature value of the solvent-quality exponent $v=0.53 .^{4}$
\end{abstract}


However, once the polymer volume fraction exceeds the "swelling volume fraction" $\phi_{s}$, above which the polymer takes on a random walk configuration on all length scales even in a good solvent, this universal scaling breaks down and the polymer conformation appears to be governed by Colby-Rubinstein's scaling laws for $\Theta$ solutions. We estimate that all polybutadiene solutions in phenyl octane (a good solvent) from Colby et al. ${ }^{5}$ are above $\phi_{s}$ and can be scaled using $\Theta$ solvent scaling laws for concentrations ranging all the way up to the melt, showing that the rheological properties of melts and solutions above $\phi_{s}$ follow the same universal behavior. In general, using the "blob" model for semidilute solutions and the Colby-Rubinstein scaling for concentrated solutions provides a way to obtain the entanglement density, which can be defined as $N / N_{e}(\phi)$ for concentrated solutions, with $N_{e}(\phi)$ the concentration-dependent number of monomers per entanglement. At a fixed value of $c / c_{e}$ or, equivalently, the entanglement density $N / N_{e}(\phi)$, linear and nonlinear rheological properties for polymer solutions collapse onto universal curves.

\subsection{Introduction}

While the rheological properties of both polymer melts and dilute polymer solutions have been extensively studied, the rheology of semidilute polymer solutions has received less attention, even though many commercially useful polymer solutions, such as polyelectrolytes, emulsions, gels, and personal care products, are semidilute. One possible reason for this is that semidilute solutions are "theoretically inconvenient" because for them both polymer-polymer and polymer-solvent interactions need to be 
considered simultaneously. However, progress can be made using the concept of the excluded volume "blob," or correlation blob, first suggested by Edwards ${ }^{6}$ and developed in detail by de Gennes. ${ }^{1}$ This model assumes that excluded volume interactions and hydrodynamic interactions are important on length scales smaller than that of the correlation blob in a good solvent, but are screened on larger length scales. Analysis of the relative contributions of thermal energy, excluded volume interactions, and entanglement interactions indicates the existence of four different polymer concentration regimes each with different concentration scaling laws governing chain configuration and/or dynamics. ${ }^{7-9}$ These four regimes are separated from each other by the three transitional concentrations $c^{*}, c_{e}$, and $c_{s}=\rho_{\text {polymer }} \phi_{s}$ with $\rho_{\text {polymer }}$ the polymer density, which are discussed shortly. While thorough investigations of thermodynamic properties, such as osmotic compressibility measured by light scattering, have been carried out, rheological properties, which are affected by hydrodynamic, frictional, and entanglement interactions, have not been quite as thoroughly studied. ${ }^{2,7}$ However, in the early 1980s, Marin et al. ${ }^{10}$ were among the first to study the universality of the linear viscoelastic behavior of concentrated polymer solutions and melts. Focusing on the similarity in rheological properties in the low frequency region of the complex moduli, they proposed time-chain length, and time-concentration superpositions in plots of $J_{N}^{0} G^{*}$ versus $\eta_{0} J_{N}^{0} \omega$, where $J_{N}^{0}$ is the plateau creep compliance, $G^{*}$ is the complex modulus, $\eta_{0}$ is the zero-shear viscosity, and $\omega$ is the frequency. Here, $J_{N}^{0}$ scales as $1 / G_{N}^{0}$ with $G_{N}^{0}$ the plateau modulus, and thus $\eta_{0} J_{N}^{0}$ can be considered to be a measure of the longest relaxation time. These superposition principles suggested that effects of chain length and concentration can be effectively subsumed into the plateau modulus and the 
relaxation time, which allows one to collapse $G^{\prime}$ and $G^{\prime \prime}$ curves for different molecular weights and concentrations into a universal plot in the low frequency region. But these early superposition methods were phenomenological, yielding simple, but unexplained, power-law relationships relating the modulus and time or frequency shift factors to the concentration or molecular weight.

About a decade ago, however, Raspaud et $a .^{2}$ presented a pioneering study of rheological scaling laws based on the “correlation blob” model by de Gennes. ${ }^{1,11,12}$ This work drew on a series of seminal papers that used light scattering to demonstrate the need for an additional concentration scaling parameter, the entanglement concentration $c_{e}$ other than the overlap concentration $c^{*} \cdot{ }^{13-15}$ Raspaud et al. measured zero-shear viscosities of three different polymer/solvent pairs and successfully placed almost all their experimental points on a single master curve of $\eta / \eta_{\text {Rouse }}=60 \times\left(c / c_{e}\right)^{3.4}$, where $\eta$ is the zero-shear viscosity of the polymer solution, $\eta_{\text {Rouse }}$ is the hypothetical Rouse viscosity for an unentangled polymer solution of the same molecular weight, $c_{e}$ is the concentration at which the entanglement effect begins, and $c$ is the concentration of polymer in weight per unit volume. Expanding on this by using data for eleven additional polymers, Heo and Larson ${ }^{3,16}$ created a master curve which for $c / c_{e}>0.5$ yielded a bestfit power law of

$$
\eta_{p} / \eta_{\text {Rouse }}=(43 \pm 2) \times\left(c / c_{e}\right)^{3.12 \pm 0.05}
$$

Here, for an entangled semidilute solution, $\eta_{p} \equiv \eta-\eta_{s}$ with $\eta_{s}$ the solvent viscosity, $\eta_{\text {Rouse }}=\eta_{s}\left(c / c_{\eta}^{*}\right)^{1 /(3 v-1)}$, where $c_{\eta}^{*} \equiv 1 /[\eta]_{0}$ with $[\eta]_{0}$ the intrinsic viscosity, and $v$ the 
excluded volume exponent. The entanglement concentration $c_{e}$ is defined as $c_{e} \equiv c_{A}^{*} n_{e}^{3 v-1}$ where $c_{A}^{*} \equiv 1 /\left(M_{w} A_{2}\right)$ with $A_{2}$ the second virial coefficient. ${ }^{2,3}$

Here, we will explore more broadly the dynamic similarity of semidilute polymer solutions at the same value of $c / c_{e}$ by comparing frequency and shear-rate-dependent viscoelastic properties. We first compare the reduced complex moduli $\left(G^{\prime} / G_{N}^{0} \& G^{\prime \prime} / G_{N}^{0}\right)$ against reduced frequency $\left(\omega \tau_{e}\right)$ of polystyrene (PS)/tricresyl phosphate (TCP) solutions with the same value of $c / c_{e}$. Here, $\tau_{e}$ is the "equilibration time", which is the Rouse time of an entanglement "tube" segment, which we will discuss in more detail below. Then, we compare the reduced zero-shear viscosity $\left(\eta_{p} / \eta_{\text {Rouse }}\right)$ and the reduced first normal stress coefficient $\left(\psi_{1} /\left(\eta_{\text {Rouse }} \tau_{e}\right)\right)$ against reduced shear rate $\left(\chi_{e}\right)$ for these solutions, again at constant values of $c / c_{e}$.

We will then discuss a transition to the concentrated solution regime, which occurs at concentrations above a "swelling" volume fraction $\phi_{s}$. In addition, we will present a procedure for determining the proportionality coefficient $K_{1}$ relating the equilibration time of the Doi-Edwards tube theory for polymer melts ${ }^{17} \tau_{e, D E}$, to the equilibration time derived from the scaling theory for entangled concentrated solutions ${ }^{8} \tau_{e, \text { scaling }}$ :

$$
\tau_{e, D E}=K_{1} \tau_{e, \text { scaling }}
$$

Through this relationship, we can plot the complex moduli of concentrated solutions and melts against the same renormalized frequency $\omega \tau_{e, D E}$ and thereby test whether entangled polymer melts are dynamically equivalent to concentrated solutions. 


\subsection{Theory}

Geometric self-similarity in molecular conformation is the foundation for the static scaling theory of semidilute polymer solutions. In a semidilute solution, whose concentration exceeds the polymer overlap concentration $c^{*}$, the "correlation blob size" $\xi$ can be defined as the distance scale below which a given polymer molecule is more likely to contact itself, due to chain connectivity, than to contact another polymer chain. Thus, the polymer conformation at small length scales, below the correlation blob size, remains the same as in a dilute solution, for which polymer excluded volume effects are important. But, as the length scale expands beyond the size of a single correlation blob, the excluded volume effect is progressively screened by interactions of the chain with other chains. Thus, inside a correlation blob, the polymer coil is swollen in a good solvent, while on length scales larger than the correlation blob, the chain conformation resembles that of a melt, for which excluded volume is screened out. Hence, the chain in a semidilute solution can be thought of as a "melt of correlation blobs" with each correlation blob acting as a rescaled monomer, and the conformation of a chain as a whole follows the random-walk formula, $R^{2} \approx(N / g) \xi^{2}$ with $R$ the end-to-end distance, $N$ the number of monomers per chain, and $g$ the number of monomers per correlation blob. ${ }^{2,11}$ Since the dynamics of polymer chains in the dilute regime are governed by the Zimm model, ${ }^{19}$ and the portion of the chain inside the correlation blob behaves as though it is in the dilute regime, the longest relaxation time of a polymer chain segment inside a correlation blob $\tau_{\text {blob }}$ should be

$$
\tau_{\text {blob }} \approx \frac{\xi^{2}}{D_{b l o b}} \approx \frac{\xi^{2}}{k_{B} T / \zeta_{b l o b}} \approx \frac{\eta_{s} \xi^{3}}{k_{B} T},
$$


where the diffusion coefficient of a correlation blob is $D_{\text {blob }} \approx k_{\mathrm{B}} T / \zeta_{\text {blob }}$, and the friction coefficient of a correlation blob is $\zeta_{\text {blob }} \approx \eta_{s} \xi$ according to the Stokes-Einstein relation. However, at length scales larger than $\xi$, excluded volume and hydrodynamic interactions should be screened out by the presence of the other chains, and so, if the chains are not entangled, their behavior should follow the Rouse theory, ${ }^{20}$ yielding the longest Rouse relaxation time,

$$
\tau_{\text {Rouse }} \approx \tau_{\text {blob }}(N / g)^{2} .
$$

In the unentangled regime, the elastic shear modulus $G_{\text {Rouse }}$ and the Rouse viscosity $\eta_{\text {Rouse }}$ are respectively

$$
G_{\text {Rouse }} \approx \frac{k_{B} T}{(N / g) \xi^{3}},
$$

and

$$
\eta_{\text {Rouse }} \approx \eta_{s}(N / g) \text {. }
$$

As the concentration increases even higher and exceeds the entanglement concentration $c_{e}$, the entanglement effect comes into play, and the dynamics of the polymer chains are then controlled by the tube diameter according to the Doi-Edwards tube model. ${ }^{17}$ Hence, the tube diameter should be controlled by the size of a correlation blob $\xi$ and the number of correlation blobs per entanglement strand $n_{e}$, and since the correlation blob is a renormalized monomer, $n_{e}$ should be almost the same as the number of monomers per entanglement $N_{e}$ for the same polymer species in melt state. ${ }^{2}$ The Rouse time required for a sub-molecule large enough to fill one "tube segment" to relax gives the time scale $\tau_{e}$. Therefore, if these three parameters, $n_{e}, \xi$, and $\tau_{e}$, are determined, we can compare the linear and nonlinear rheology of different semidilute linear polymer solutions by 
using $\xi$ and $n_{e}$ to normalize the modulus, and $\tau_{e}$ to normalize the frequency (or shear rate). Here, $n_{e} \xi^{3}$ is related to the plateau modulus of a semidilute solution by the classical rubber elasticity theory ${ }^{18}$ as

$$
G_{N}^{0} \approx k_{B} T /\left(n_{e} \xi^{3}\right) .
$$

Because correlation blobs in semidilute solutions fill the space as monomers in melts do, the volume fraction of polymer in the solution $\phi$ is equal to the volume fraction of monomers in a single correlation blob of volume $\xi^{3}$ :

$$
\phi \approx g b^{3} / \xi^{3},
$$

where $b$ is the statistical segment length, and $\xi \approx b g^{v}$ from the expression for the radius of a coil containing $g$ monomers, while $v$ is the excluded volume exponent for the polymer/solvent pair. Thus, the above implies that $g$ scales as $\phi^{1 /(1-3 v)}$ and $\xi$ scales as $\phi^{\nu /(1-3 \nu)}$, leading to

$$
G_{N}^{0}(\phi) \approx G_{N}^{0}(1) \phi^{3 v /(3 v-1)},
$$

where $G_{N}^{0}(1)$ is the plateau modulus of the melt.

This scaling exponent for $G_{N}^{0}$ is identical to that for the dependence of osmotic pressure $\pi$ on the volume fraction of a semidilute solution as shown in Figure 3.1:

$$
\pi \sim \phi^{3 v /(3 v-1)} .
$$




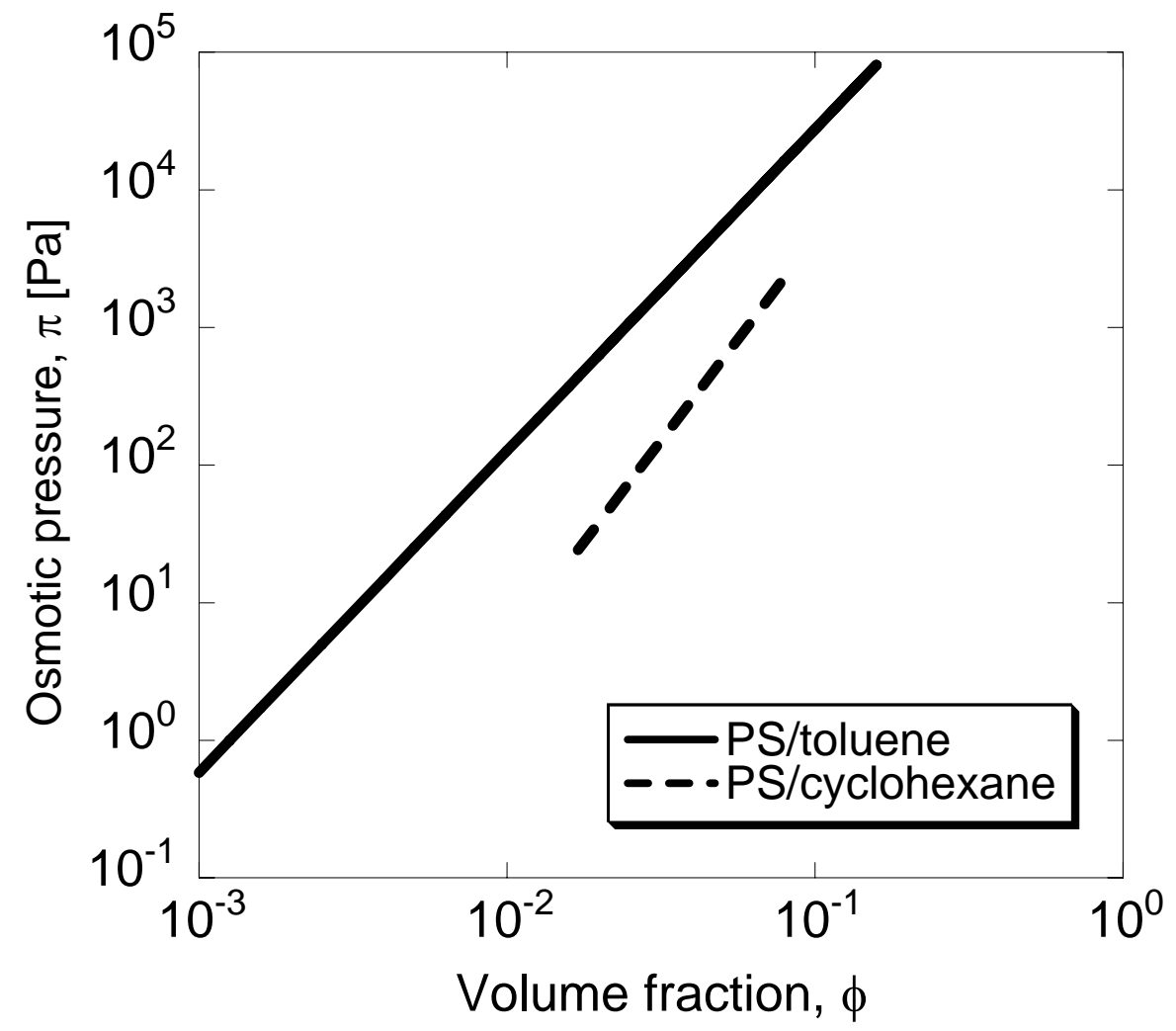

Figure 3.1. Osmotic pressure for polystyrene (PS) in toluene at $25^{\circ} \mathrm{C}$ (a good solvent) and in cyclohexane at the $\Theta$ temperature, $35^{\circ} \mathrm{C}$. Solid lines denote experimental data of PS/toluene ${ }^{22}$, and dashed lines denote those of PS/cyclohexane. ${ }^{21}$

Thus, if the modulus follows the same concentration scaling law as the osmotic pressure, the "dilution exponent" $\alpha$ in the relationship $G_{N}^{0} \sim \phi^{1+\alpha}$ should range from 1.31 for good solvents to 2.0 for $\Theta$ solvents. However, many experimental measurements on polymer solutions $^{7,23--26}$ seem to support the conjecture of Colby and Rubinstein ${ }^{12}$ that the “dilution exponent” $\alpha$ in the relationship $G_{N}^{0} \sim \phi^{1+\alpha}$ is $4 / 3$ for $\Theta$ solvents, which gives no 
practical difference from the value, $\alpha=1 /(3 v-1)=1.31$ for good solvents with $v=0.588$; see Figure 3.2.

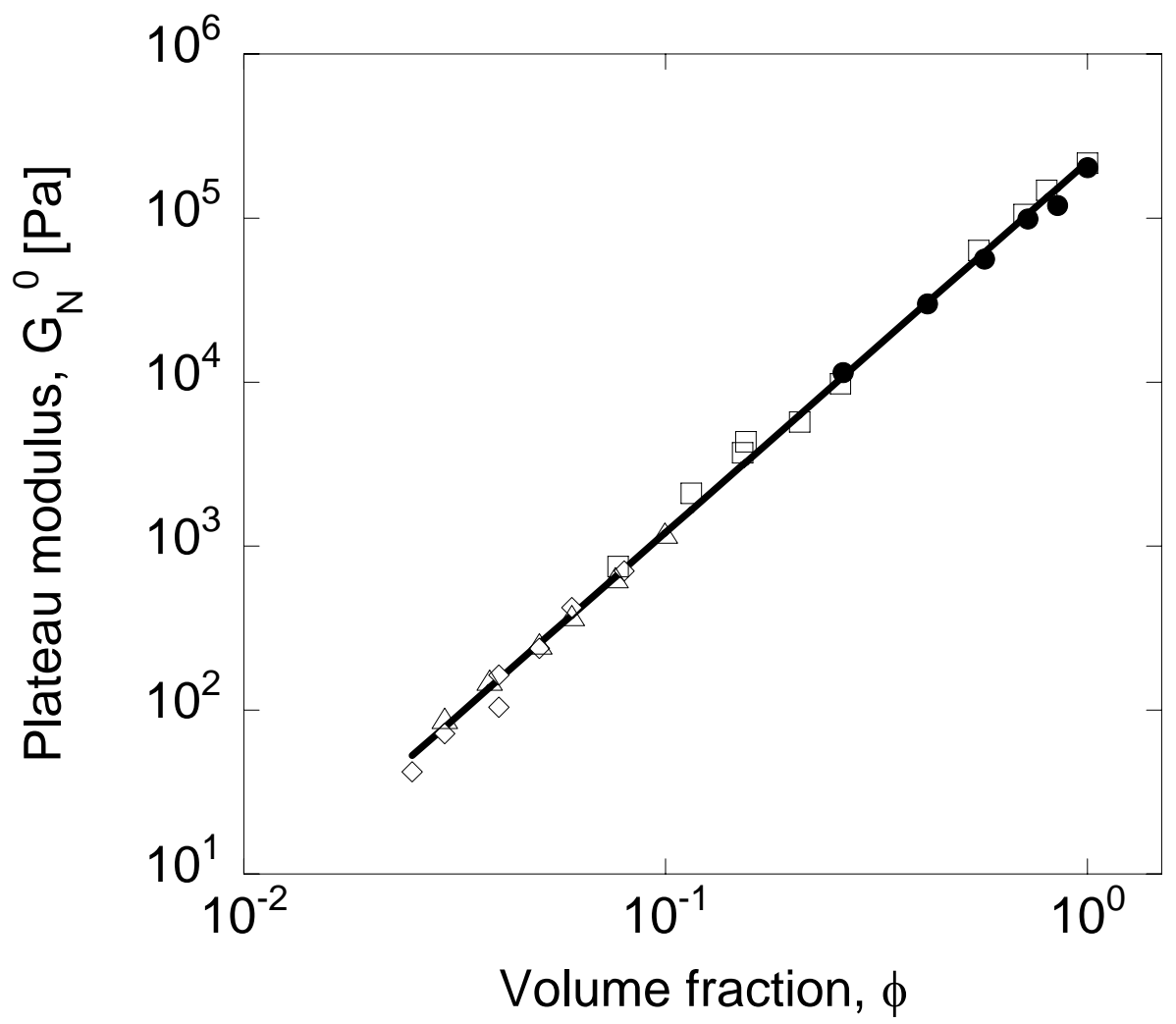

Figure 3.2. Plateau modulus against volume fraction of nearly monodisperse polystyrene solutions in Aroclor $1248^{25,26}$ (open diamonds and open triangles), n-butyl benzyl phthalate $^{23}$ (open squares), and in tricresyl phosphate ${ }^{24}$ (filled circles). The line is a leastsquares fit to all data.

These results, and experimental data on polymer solutions of various solvent qualities, suggest that the dilution exponent $\alpha$ is almost independent of solvent quality. But many of the experiments that were within the semidilute regime and believed to support the "universality" of the dilution exponent $\alpha$ (such as polystyrene solutions in Aroclor 1248 
in Figure 3.2, for which $v=0.567$ ) are not useful for exploring the effect of solvent quality on the plateau modulus because they are too close to being in the good-solvent limit ( $v \cong 0.588$ ), for which the blob theory gives a dilution exponent of 1.31 $(=1 /(3 v-1))$, essentially identical to the value proposed for a theta solvent by Colby and Rubinstein. Thus, for such polymer/solvent pairs, there is no strong difference between the Colby-Rubinstein scaling and the predictions of the blob theory. It is true that there are some solutions studied that have intermediate solvent quality; an example is polystyrene (PS) solutions in tricresyl phosphate (TCP), for which $v=0.53$, and therefore $1 /(3 v-1)=1.7$. Although this value of $\alpha$ is different enough from $\alpha=1.31$ to provide a good test of the de Gennes blob-theory scaling of plateau modulus with concentration, the concentrations studied so far lie mostly outside of the semidilute regime, and are instead within the concentrated regime ( $\phi \geq 0.25$; see filled circles in Figure 3.2), where the blob theory does not apply. Hence, a thorough test of the Colby-Rubinstein conjecture requires that we measure the dependence of the plateau modulus on polymer concentration in the semidilute (i.e., relatively low concentration) regime using a polymer in a marginally good solvent. We will discuss this further in the Results and Discussion section.

The characteristic time constant $\tau_{e, \text { scaling }}$, which, after the plateau modulus, is the second essential parameter for scaling, corresponds to the Rouse relaxation time of an entanglement strand. We can use Eq. 4, namely $\tau_{\text {Rouse }} \approx \tau_{\text {blob }}(N / g)^{2}$, to obtain an expression for the equilibration time from the correlation blob relaxation time:

$$
\tau_{e, \text { scaling }}(\phi) \approx \tau_{\text {blob }}\left[N_{e}(\phi) / g(\phi)\right]^{2} \approx \frac{\eta_{s}}{k_{B} T} \xi(\phi)^{3}\left[N_{e}(\phi) / g(\phi)\right]^{2} \text {, }
$$


where $N_{e}(\phi)$ is the number of monomers per entanglement strand at volume fraction $\phi$, and $N_{e}(\phi) / g(\phi)$ is essentially equal to $n_{e},{ }^{2}$ the number of correlation blobs needed for an entanglement. Since the radius of gyration of a polymer chain in a dilute solution scales with the number of monomers per chain as $R_{g} \approx N^{v}$, the correlation blob size $\xi$ at a concentration $c$ above the overlap concentration $c^{*}$ also follows this relationship $\xi \approx g^{v}$, where $c^{*} \approx N / R_{g}^{3}$ and $c \approx g / \xi^{3}$. Thus, $c / c^{*} \approx \frac{g / \xi^{3}}{N / R_{g}^{3}} \approx(N / g)^{3 v-1}$ since $\xi / R_{g} \approx(g / N)^{v}$, leading to $\xi \approx R_{g}\left(C / c^{*}\right)^{v /(1-3 v)}$. ( $R_{g}$ is the radius of gyration of the polymer in dilute solution, not in semidilute solution.) Therefore, from Eq. 11, we can write the equilibration time of a semidilute solution in terms of $c / c^{*}$ as

$$
\tau_{e, \text { scaling }}=\frac{\eta_{s}}{k_{B} T} R_{g}^{3}\left(c / c^{*}\right)^{3 v /(1-3 v)} n_{e}^{2} .
$$

In the Results and Discussion section, we will plot our experimental normalized linear complex moduli, $G^{\prime} / G_{N}^{0}, G^{\prime \prime} / G_{N}^{0}$ against $\omega \tau_{e}$, and our experimental nonlinear normalized steady shear functions, $\eta_{p} / \eta_{\text {Rouse }}, \psi_{1} /\left(\eta_{\text {Rouse }} \tau_{e}\right)$ against $\tau_{e}$, where $\psi_{1}$ is the first normal stress coefficient, and $\gamma$ is the shear rate.

\subsection{Experimental Materials and Methods}

\subsubsection{Materials}

We purchased nearly monodisperse polystyrene (PS) samples from Tosoh Bioscience and Polymer Laboratories; the part number (batch number) and weight-average molecular 
weight of each sample are given in Table 3.1. Tricresyl phosphate (TCP) is used as a marginally good solvent for PS with excluded volume exponent $v=0.53 .^{4}$

Table 3.1. Nominal and actual molecular weights of polystyrene samples

\begin{tabular}{|c|c|c|c|c|}
\hline Vendor & $\begin{array}{c}\text { Part No. } \\
(\text { Batch No. })\end{array}$ & $\begin{array}{c}\text { Nominal } M_{w} \\
\left(10^{6} \mathrm{~g} / \mathrm{mol}\right)\end{array}$ & $\begin{array}{c}\text { Actual } M_{w} \\
\left(10^{6} \mathrm{~g} / \mathrm{mol}\right)\end{array}$ & $\begin{array}{c}\text { Standard Error } \\
\left(10^{6} \mathrm{~g} / \mathrm{mol}\right)\end{array}$ \\
\hline \multirow{3}{*}{$\begin{array}{c}\text { Tosoh } \\
\text { Bioscience }\end{array}$} & F-128 & 1.09 & 1.28 & 0.01 \\
\cline { 2 - 5 } & F-288 & 2.11 & 2.53 & 0.08 \\
\cline { 2 - 5 } & F-550 & 5.48 & 6.62 & 0.06 \\
\hline $\begin{array}{c}\text { Polymer } \\
\text { Laboratories }\end{array}$ & $20144-13$ & 2.35 & 2.68 & 0.06 \\
\cline { 2 - 5 } & $20146-15$ & 4.74 & 5.56 & 0.10 \\
\hline
\end{tabular}

* All weight-average molecular weights were measured with a Wyatt DAWN EOS static light scattering goniometer.

\subsubsection{Sample Preparation}

\subsubsection{Weight-average Molecular Weight Measurements}

We selected six different reduced concentrations within the semidilute regime, namely $c / c_{e}=0.77,1.22,1.65,1.85,2.50$, and 3.00 , where $c_{e}$ is related to molecular weight through $c_{e}=c_{A}^{*} n_{e}^{3 v-1}$ where the overlap concentration here is based on the polymer thermal radius $R_{t}=\left[3 A_{2} M_{w}^{2} /\left(16 \pi N_{A}\right)\right]^{1 / 3}, 3,31$ and in turn, $c_{A}^{*} \equiv 1 /\left(M_{w} A_{2}\right) \sim M_{w}^{1-3 v}, 2,3$ since $R_{t}$ is proportional to the radius of gyration. ${ }^{3,32}$ Hence, determining the correct molecular weight is an essential step prior to measurements. Since we use polystyrene samples from 
two different vendors, we need to check the absolute weight-average molecular weights of these samples using the same device by the same person to ensure consistency. Values of $M_{w}$ in Table 1 represent the absolute molecular weights measured by small angle static light scattering with a Wyatt DAWN EOS; see Figure 3.3.

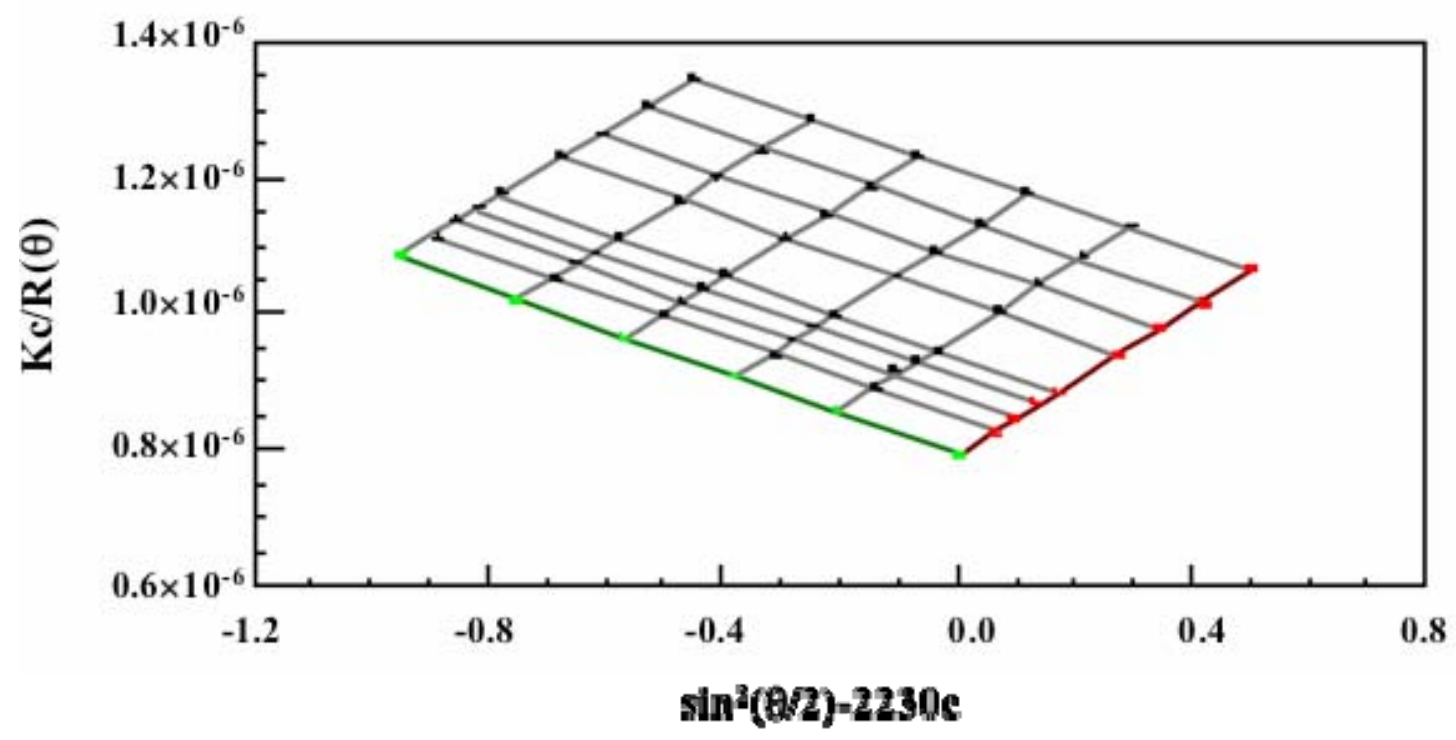

Figure 3.3. Sample Zimm plot of $1.28 \times 10^{6} \mathrm{~g} / \mathrm{mol}$ polystyrene in toluene at room temperature. Here, $\theta, c, R(\theta)$, and $K$ respectively denote the detection angle, the concentration of a sample in $\mathrm{g} / \mathrm{ml}$, the excess Rayleigh ratio, and $K=\frac{4 \pi^{2}}{N_{A}} \frac{n_{0}^{2}}{\lambda_{0}^{4}}\left(\frac{d n}{d c}\right)^{2}$ with $N_{A}$ the Avogadro's number, $n_{0}$ the solvent refractive index, $\lambda_{0}$ the vacuum wavelength of incident light, and $d n / d c$ the refractive index increment. Note that a negative "stretch factor," -2230, has been used for enhanced interpretability. We employed a "Berry plot" for $2.53 \times 10^{6} \mathrm{~g} / \mathrm{mol}$ and $6.62 \times 10^{6} \mathrm{~g} / \mathrm{mol}$ polystyrene, since this method of plotting is recommended for polymers with the molecular weight higher than 1 million $\mathrm{g} / \mathrm{mol}$.

\subsubsection{Preparation of Solutions}

We prepared the samples by mixing PS and TCP in dichloromethane (DCM). Even though TCP is a thermodynamically good solvent for PS, dissolving PS in TCP is a kinetically slow process due to relatively high viscosity of TCP $\left(58 \mathrm{cP}\right.$ at $\left.25^{\circ} \mathrm{C}\right)$. For this 
reason, we use DCM as a co-solvent that helps PS chains dissolve faster by increasing the solvent volume, and by reducing the viscosity of the solution. After complete blending of these three components on a roller overnight, we evaporate most of DCM in a fume hood and completely remove the remaining DCM by placing the samples in a vacuum oven for about three weeks until less than $0.001 \mathrm{~g}$ of weight loss occurs over two consecutive days.

\subsubsection{Measuring Rheological Properties with Bubble-free Samples}

We used an ARES LS (Rheometrics) with $25.0 \mathrm{~mm}$ diameter parallel plates to obtain

the linear and nonlinear viscoelastic properties of semidilute PS/TCP solutions at a temperature of $25.0 \pm 0.1^{\circ} \mathrm{C}$ controlled using a water bath. Due to the relatively long relaxation times of PS/TCP solutions, when a sample was removed from a container and loaded on the bottom plate of the fixture, air bubbles easily got trapped inside the sample. To remove them, we first placed the bottom plate, on which the sample was loaded, into a vacuum oven and held it at $60^{\circ} \mathrm{C}$ for several hours. Then, we applied a vacuum to burst any remaining bubbles. In addition, air bubbles can become trapped in a sample when the upper plate is driven into the sample when setting the gap. Lowering the upper plate faster than the relaxation time of a sample does not give the polymer chains enough time to rearrange themselves under stress, which creates an uneven surface, capturing air between upper plate and the surface of the sample. Thus, additional care was taken when lowering the upper plate. 


\subsection{Results and Discussion}

\subsubsection{Nearly Monodisperse Polystyrene Solutions}

Here, we test the dynamic similarities of semidilute nearly monodisperse polystyrenes (PS) of three different molecular weights in tricresyl phosphte (TCP). Tables 3.2 and 3.3 list all necessary parameters for computing the Rouse viscosity $\eta_{\text {Rouse }}(\phi)$, the plateau modulus $G_{N}^{0}(\phi)$, and the equilibration time $\tau_{e, \text { scaling }}(\phi)$ of each solution. Here, $\eta_{\text {Rouse }}(\phi)$ is calculated from $\eta_{\text {Rouse }}(\phi)=\eta_{s}\left(c / c_{\eta}^{*}\right)^{1 /(3 v-1)}$ with $c_{\eta}^{*} \equiv 1 /[\eta]_{0}$, where $[\eta]_{0}$ is the intrinsic viscosity of the polymer.

Table 3.2. The parameters of polystyrene (PS)/tricresyl phosphate (TCP) solutions at $25^{\circ} \mathrm{C}$; the zero-shear viscosity of TCP $\eta_{s}$, the number of correlation blobs per entanglement for PS/TCP solutions $n_{e}$, the excluded volume exponent for PS/TCP solutions $v$, the dilution exponent $\alpha$ for PS/TCP solutions, the plateau modulus of PS melt at $180^{\circ} \mathrm{C} G_{N}^{0}(1)$, and the densities of PS and TCP at $25^{\circ} \mathrm{C}, \rho_{P S}$ and $\rho_{T C P}$.

\begin{tabular}{|c|c|c|c|c|c|c|}
\hline$\eta_{s}$ & $n_{e}{ }^{\mathrm{a}}$ & $v^{\mathrm{b}}$ & $\alpha^{\mathrm{c}}$ & $G_{N}^{0}(1)^{\mathrm{d}}$ & $\rho_{P S}$ & $\rho_{T C P}$ \\
$\mathrm{~Pa} \cdot \mathrm{s}$ & & & $\mathrm{Pa}$ & $\mathrm{g} / \mathrm{L}$ & $\mathrm{g} / \mathrm{L}$ \\
\hline 0.058 & 185 & 0.53 & 1.7 & $2.23 \times 10^{5}$ & 1060 & 1160 \\
\hline
\end{tabular}

${ }^{\text {a }}$ ref. 2

${ }^{\mathrm{b}}$ the excluded volume exponent is calculated as $v=(a+1) / 3$ from the Mark-Houwink exponent, $a$ in $[\eta]_{0}=4.2 \times 10^{-5} M_{w}^{0.59}(\mathrm{~L} / \mathrm{g})$ from data in the molecular weight range of $2 \times 10^{5}-2 \times 10^{6} \mathrm{~g} / \mathrm{mol}^{4}{ }^{4} \mathrm{We}$ assume that this same excluded volume exponent applies to our samples, including those with molecular weight higher than two million g/mol.

c $\alpha$ is computed from the relationship, $\alpha=1 /(3 v-1)$.

${ }^{\mathrm{d}} G_{N}^{0}(1)$ is calculated from $G_{N}^{0}(1)=(4 / 5) \rho R T / M_{e}{ }^{28}$ 
Table 3.3. Parameters of polystyrene (PS) of three different molecular weights in tricresyl phosphate (TCP): the weight-average molecular weight $M_{w}$, the intrinsic viscosity $[\eta]_{0}$, the radius of gyration $R_{g}$, the overlap concentration $c^{*}$, the second virial coefficient multiplied by the molecular weight $M_{w} A_{2}$, and the entanglement concentration $c_{e}$ of each PS sample.

\begin{tabular}{|c|c|c|c|c|c|}
\hline$M_{w}$ & {$[\eta]_{0}{ }^{\mathrm{a}}$} & $R_{g}{ }^{\mathrm{b}}$ & $c^{{ }^{*} \mathrm{c}}$ & $M_{w} A_{2}{ }^{\mathrm{d}}$ & $c_{e}{ }^{\mathrm{e}}$ \\
\hline $\mathrm{g} / \mathrm{mol}$ & $\mathrm{L} / \mathrm{g}$ & $\mathrm{Nm}$ & $\mathrm{g} / \mathrm{L}$ & $\mathrm{L} / \mathrm{g}$ & $\mathrm{g} / \mathrm{L}$ \\
\hline $2.28 \times 10^{6}$ & 0.168 & 43.9 & 25.2 & 0.258 & 84.3 \\
\hline $6.62 \times 10^{6}$ & 0.444 & 104.8 & 9.55 & 0.680 & 56.4 \\
\hline
\end{tabular}

${ }^{\mathrm{a}}[\eta]_{0}=4.2 \times 10^{-5} M_{w}^{0.59}(\mathrm{~L} / \mathrm{g})^{4}$

${ }^{\mathrm{b}} R_{v} / R_{g}=0.74$ for linear PS solutions over the range $10^{5} \leq M_{w} \leq 10^{6} \cdot{ }^{31}$ Here, the viscometric radius of the polymer chain $R_{v}$ is calculated as $R_{v}=\left(\frac{3[\eta]_{0} M_{w}}{10 \pi N_{A}}\right)^{1 / 3}$ with $N_{A}$ Avogadro's number, and $R_{g}=\left(\frac{3[\eta]_{0} M_{w}}{10 \pi N_{A}}\right)^{1 / 3} / 0.74^{3}$

${ }^{\mathrm{c}} C^{*} \equiv \frac{M_{w}}{N_{A} R_{g}^{3}}$

${ }^{\mathrm{d}} M_{w} A_{2}=6.5 / c^{*}$ for PS in good solvents ${ }^{2}$

${ }^{\mathrm{e}} c_{e} \equiv\left(1 / M_{w} A_{2}\right) n_{e}^{3 v-1} \cdot 2,3$

Now, we can compare rheological data of PS/TCP solutions, using $\tau_{e \text {,scaling }}$ as a “relative” equilibration time for comparing data for PS/TCP solutions with varying concentration and molecular weight. We measured linear rheological properties for $c / c_{e}=0.77,1.22,1.65,1.85,2.50$, and 3.00 , which are tabulated in Table 3.4 and shown in Figure 3.4. 
Table 3.4. Volume fraction $\phi$, equilibration time $\tau_{e, \text { scaling }}$ calculated from Eq. (12), and the plateau modulus $G_{N}^{0}(\phi)=G_{N}^{0}(1) \phi^{1+\alpha}$ for each PS/TCP solution at various molecular weights $M_{w}$, and reduced concentrations $c / c_{e}$.

\begin{tabular}{|c|c|c|c|c|c|c|c|c|c|c|c|}
\hline \multicolumn{6}{|c|}{ Nominal $c / c_{e}=0.77$} & \multicolumn{6}{|c|}{ Nominal $c / c_{e}=1.22$} \\
\hline $\begin{array}{c}M_{w} \\
\mathrm{~g} / \mathrm{mol}\end{array}$ & $\phi$ & $\begin{array}{c}\text { Actual } \\
c / c_{e}\end{array}$ & $\tau_{e, \text { scaling }}$ & $\begin{array}{c}G_{N}^{0}(\phi) \\
\mathrm{Pa}\end{array}$ & $\begin{array}{c}\eta_{\text {Rouse }} \\
\mathrm{Pa}\end{array}$ & $\begin{array}{c}M_{w} \\
\mathrm{~g} / \mathrm{mol}\end{array}$ & $\phi$ & $\begin{array}{c}\text { Actual } \\
C / C_{e}\end{array}$ & $\begin{array}{c}\tau_{e, \text { scaling }} \\
\mathrm{s}\end{array}$ & $\begin{array}{c}G_{N}^{0}(\phi) \\
\mathrm{Pa}\end{array}$ & $\begin{array}{c}\eta_{\text {Rouse }} \\
\mathrm{Pa}\end{array}$ \\
\hline $1.28 \times 10^{6}$ & 0.061 & 0.77 & 0.0206 & 118 & 3.33 & $1.28 \times 10^{6}$ & 0.094 & 1.18 & 0.0065 & 373 & 6.87 \\
\hline $2.53 \times 10^{6}$ & 0.040 & 0.76 & 0.0626 & 39 & 3.27 & $2.53 \times 10^{6}$ & 0.065 & 1.22 & 0.0176 & 138 & 7.27 \\
\hline $6.62 \times 10^{6}$ & 0.024 & 0.78 & 0.2711 & 40 & 3.41 & $6.62 \times 10^{6}$ & 0.036 & 1.20 & 0.0844 & 29 & 7.10 \\
\hline \multicolumn{6}{|c|}{ Nominal $c / c_{e}=1.65$} & \multicolumn{6}{|c|}{ Nominal $c / C_{e}=1.85$} \\
\hline $\begin{array}{c}M_{w} \\
g / m o l\end{array}$ & $\phi$ & $\begin{array}{c}\text { Actual } \\
c / c_{e}\end{array}$ & $\tau_{e, \text { scaling }}$ & \multicolumn{2}{|c|}{$G_{N}^{0}(\phi)$} & $\begin{array}{c}M_{w} \\
\mathrm{~g} / \mathrm{mol}\end{array}$ & $\phi$ & $\begin{array}{c}\text { Actual } \\
c / c_{e}\end{array}$ & $\begin{array}{c}\tau_{e, \text { scaling }} \\
\mathrm{S}\end{array}$ & \multicolumn{2}{|c|}{$G_{N}^{0}(\phi)$} \\
\hline $1.28 \times 10^{6}$ & 0.131 & 1.65 & 0.0026 & & 925 & $1.28 \times 10^{6}$ & 0.146 & 1.84 & 0.0020 & \multicolumn{2}{|r|}{1,237} \\
\hline $2.53 \times 10^{6}$ & 0.089 & 1.67 & 0.0075 & & 324 & $2.53 \times 10^{6}$ & 0.098 & 1.84 & 0.0058 & \multicolumn{2}{|r|}{419} \\
\hline $6.62 \times 10^{6}$ & 0.049 & 1.63 & 0.0369 & & 66 & $6.62 \times 10^{6}$ & 0.056 & 1.86 & 0.0259 & \multicolumn{2}{|r|}{94} \\
\hline \multicolumn{6}{|c|}{ Nominal $c / c_{e}=2.50$} & \multicolumn{6}{|c|}{ Nominal $c / c_{e}=3.00$} \\
\hline $\begin{array}{c}M_{w} \\
\mathrm{~g} / \mathrm{mol}\end{array}$ & $\phi$ & $\begin{array}{c}\text { Actual } \\
c / c_{e}\end{array}$ & $\begin{array}{c}\tau_{e, \text { scaling }} \\
\mathrm{s}\end{array}$ & $\begin{array}{r}G_{N}^{0}( \\
\text { Pa }\end{array}$ & & $\begin{array}{c}M_{w} \\
\mathrm{~g} / \mathrm{mol}\end{array}$ & $\phi$ & $\begin{array}{l}\text { Actual } \\
c / c_{e}\end{array}$ & $\begin{array}{c}\tau_{e, \text { scaling }} \\
\mathrm{s}\end{array}$ & \multicolumn{2}{|c|}{$G_{N}^{0}(\phi)$} \\
\hline $1.28 \times 10^{6}$ & 0.199 & 2.50 & 0.0009 & & 6,398 & $1.28 \times 10^{6}$ & 0.239 & 3.00 & 0.0005 & \multicolumn{2}{|r|}{9,547} \\
\hline $2.53 \times 10^{6}$ & 0.133 & 2.50 & 0.0025 & & 2,632 & $2.53 \times 10^{6}$ & 0.160 & 3.00 & 0.0016 & \multicolumn{2}{|r|}{3,937} \\
\hline $6.62 \times 10^{6}$ & 0.075 & 2.50 & 0.0117 & & 756 & $6.62 \times 10^{6}$ & 0.091 & 3.00 & 0.0071 & \multicolumn{2}{|r|}{1,131} \\
\hline
\end{tabular}


(a) Nominal $c / c_{e}=0.77$
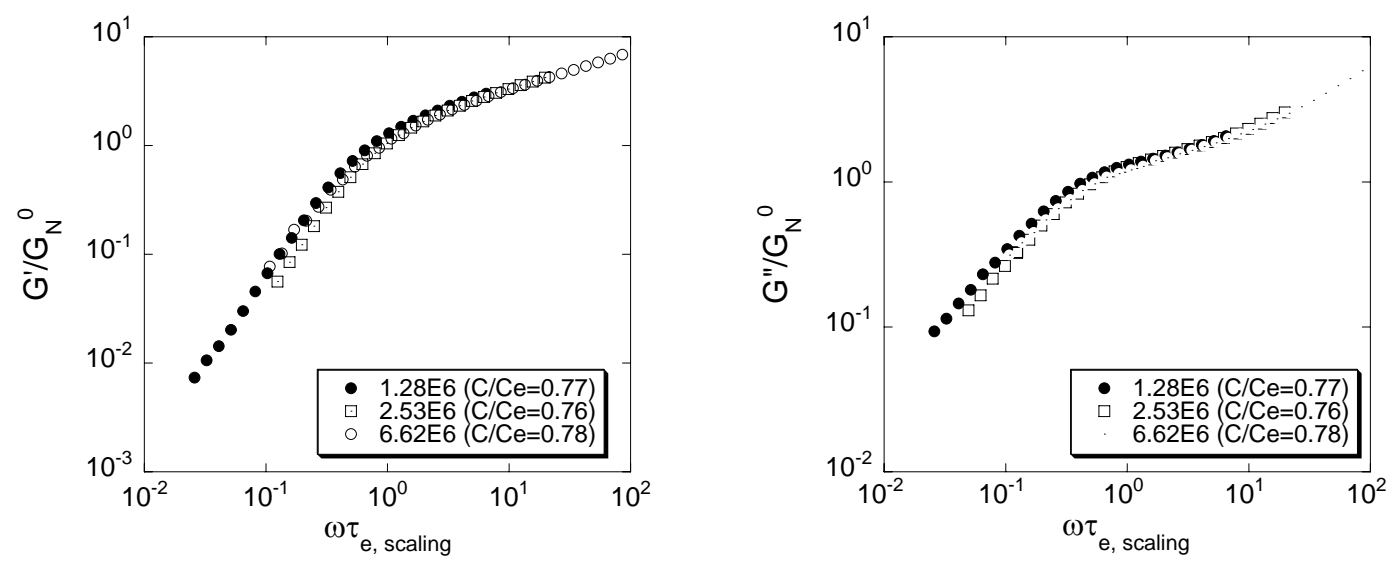

(b) Nominal $c / c_{e}=1.20$
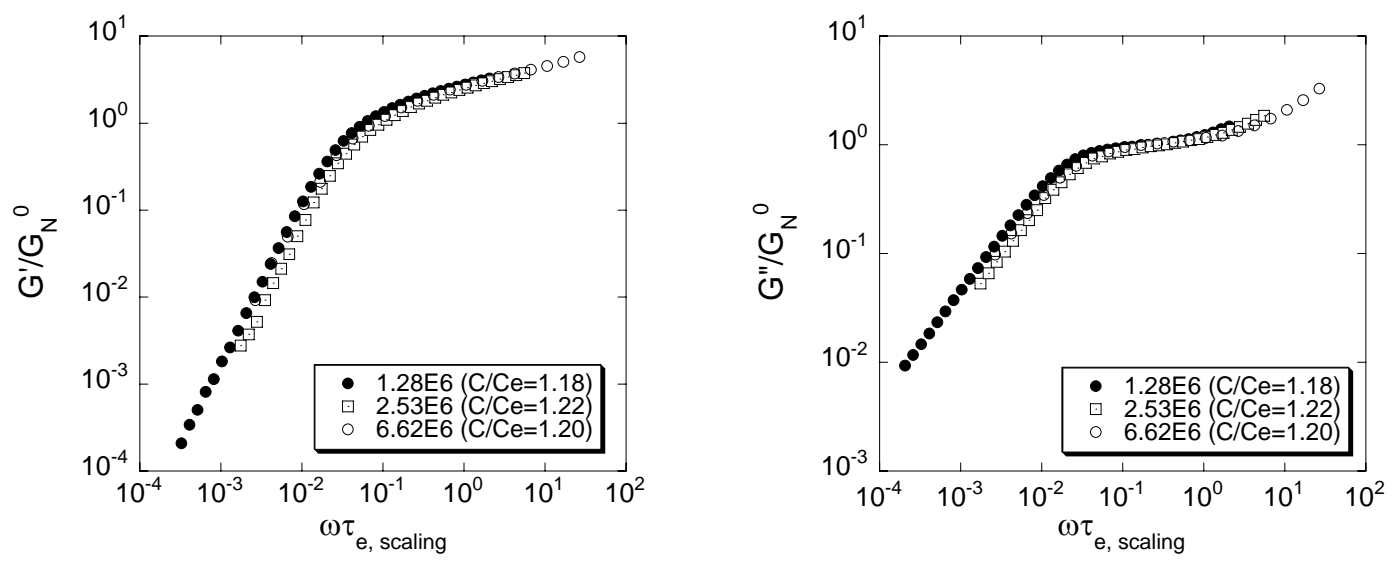
(c) Nominal $c / c_{e}=1.65$
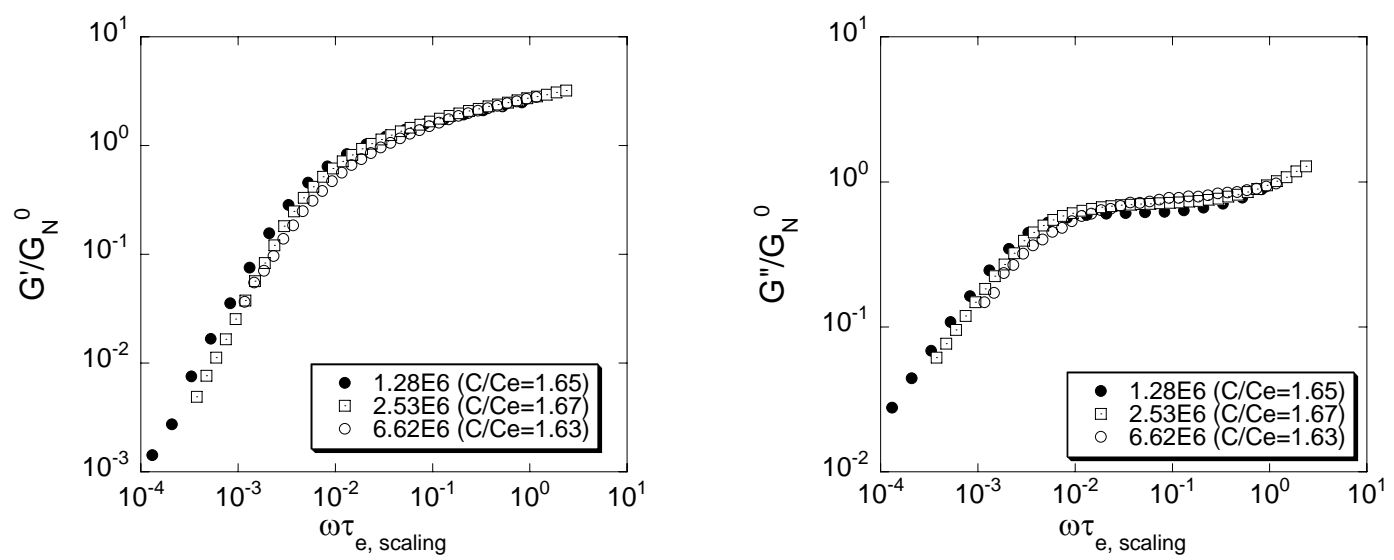

(d) Nominal $c / c_{e}=1.85$
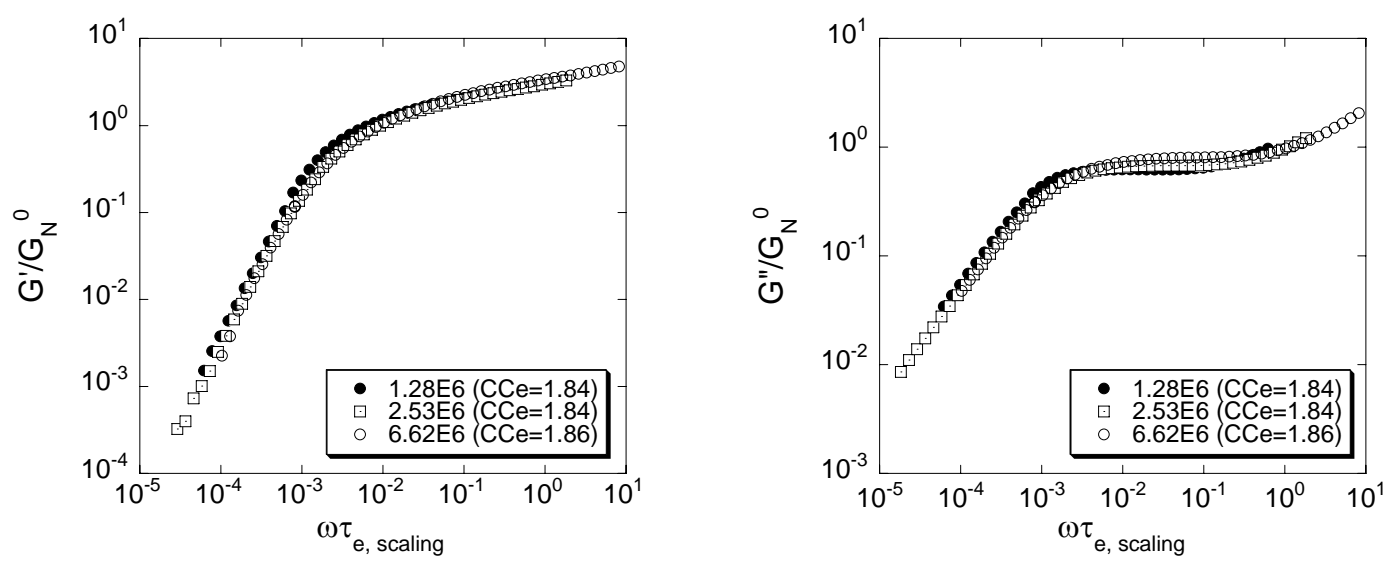
(e) Nominal $c / c_{e}=2.50$
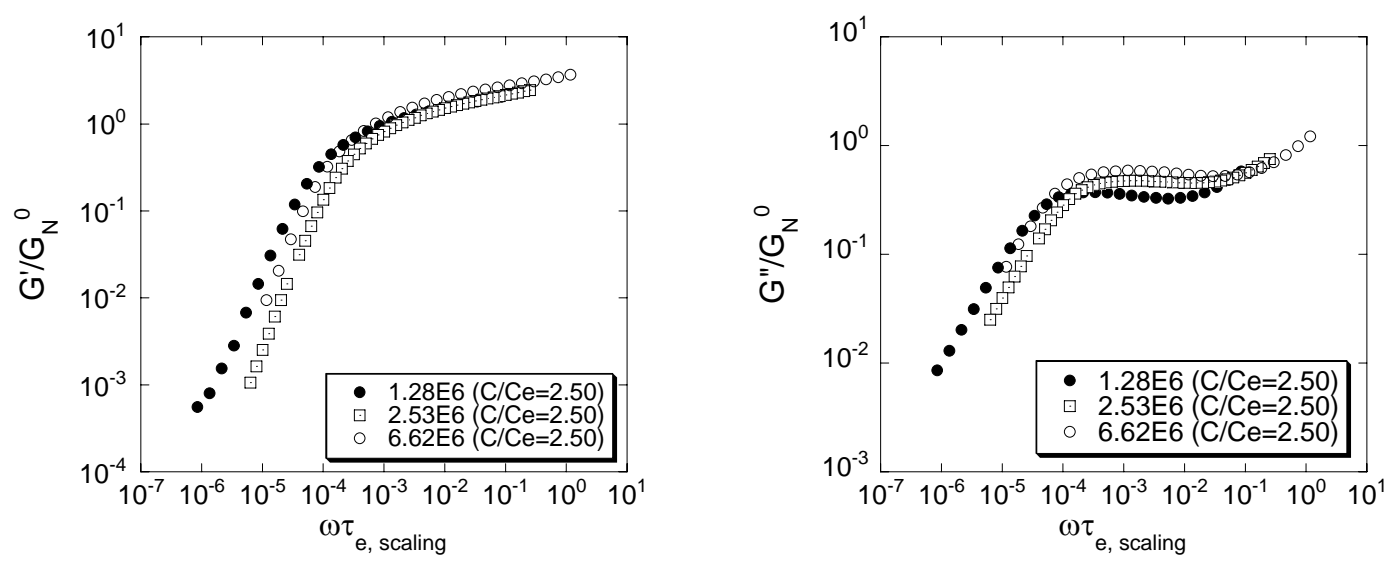

(f) Nominal $c / c_{e}=3.00$
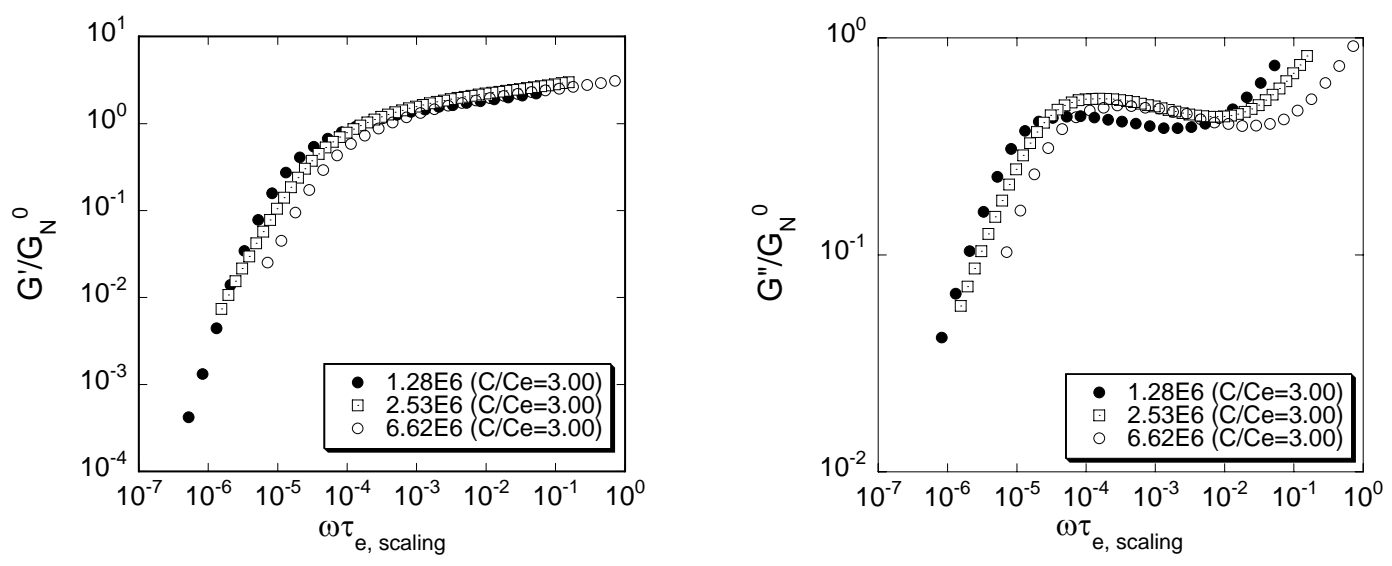

Figure 3.4. Plots testing "universal scaling" of the linear viscoelastic properties of semidilute monodisperse polystyrene (PS)/tricresyl phosphate (TCP) solutions. In each plot, normalized storage and loss moduli, $G^{\prime} / G_{N}^{0}$ and $G^{\prime \prime} / G_{N}^{0}$ are plotted against normalized frequency, $\omega \tau_{e \text {, scaling }}$ at a given nominal $c / c_{e}$. The small differences in values of $c / c_{e}$ within each figure are recorded in the legends. Filled circles, open squares, and 
open circles respectively represent the storage and loss moduli of $1.28 \times 10^{6} \mathrm{~g} / \mathrm{mol}$, $2.53 \times 10^{6} \mathrm{~g} / \mathrm{mol}$, and $6.62 \times 10^{6} \mathrm{~g} / \mathrm{mol}$ PS solutions.

We were able to measure nonlinear rheological functions for only $c / c_{e}=0.77$ and 1.22 due to edge fracture at volume fractions higher than about $10 \%{ }^{32,33}$ Even so, for these solutions, excellent superposition is obtained in plots of the nonlinear functions $\psi_{1} /\left(\eta_{\text {Rouse }} \tau_{e, \text { scaling }}\right)$, and $\eta_{p} / \eta_{\text {Rouse }}$ against $\bar{t}_{e, \text { scaling }}$, except for low shear-rate regions; see Figure 5. This can be attributed to poor torque values since the lower limit of the transducer (Force Rebalance Transducer, 2K FRTN1 for high range) is $2 \mathrm{~g} \cdot \mathrm{cm}$.
(a) Nominal $c / c_{e}=0.77$
(b) Nominal $c / c_{e}=1.22$
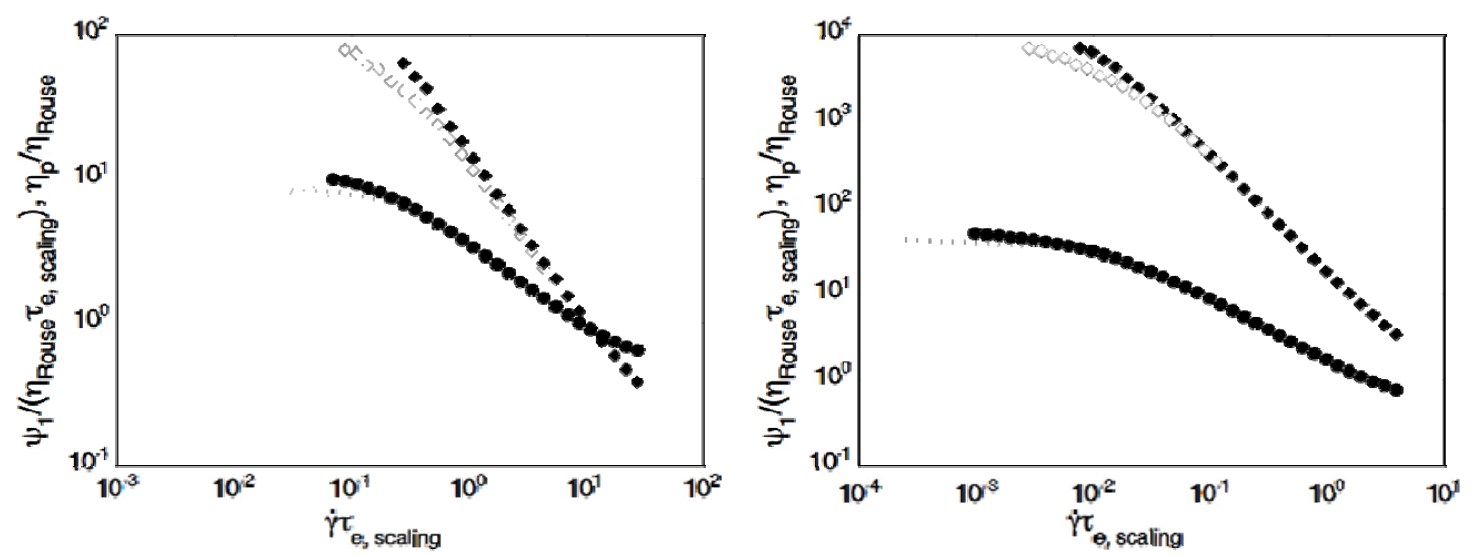

Figure 3.5. "Universal scaling" of the nonlinear viscoelastic properties of semidilute monodisperse PS/TCP solutions. The normalized first normal stress coefficient $\psi_{1} /\left(\eta_{\text {Rouse }} \tau_{e, \text { scaling }}\right)$ and the normalized steady shear viscosity $\eta_{p} / \eta_{\text {Rouse }}$ are plotted against the normalized shear rate, $\dot{x}_{e, \text { scaling }}$, at two nominal values of $c / c_{e}$, where the Rouse viscosities for these two concentrations are computed from $\eta_{\text {Rouse }}=\eta_{s}\left(c[\eta]_{0}\right)^{1 /(3 v-1)}$ as given earlier in this section, and are tabulated in Table 3.4. Filled and open diamonds, respectively, represent the normalized first normal stress difference coefficients of $6.62 \times 10^{6} \mathrm{~g} / \mathrm{mol}$ and $2.53 \times 10^{6} \mathrm{~g} / \mathrm{mol}$ PS solutions. Filled and open circles, respectively, 
represent the normalized steady shear viscosities of $6.62 \times 10^{6} \mathrm{~g} / \mathrm{mol}$ and $2.53 \times 10^{6} \mathrm{~g} / \mathrm{mol}$ PS solutions.

In Figure 3.4, $G^{\prime} / G_{N}^{0}$ and $G^{\prime \prime} / G_{N}^{0}$ are plotted against $\omega \tau_{e \text {,scaling }}$ for PS of three different molecular weights in TCP at various $c / c_{e}$ 's, ranging from 0.77 to 3.00. At relatively low $c / c_{e}$, namely $c / c_{e}=0.77,1.22,1.65$, and 1.85 , we have excellent collapse with only slight discrepancies in the terminal region. However, the shapes of the $G^{\prime \prime} / G_{N}^{0}$ curves between the terminal and the high-frequency regions begin to deviate from each other with increasing $c / c_{e}$, indicating the gradual breakdown of the universality based on the "blob" model within the semidilute regime. Thus, the superposition of the complex moduli starts to break down when the reduced concentration exceeds $c / c_{e}=2.0$ for nearly monodisperse PS/TCP solutions. This breakdown of "universal” scaling for semidilute solutions is also observed with the binary blends of two different molecular weights of PS in TCP, as will be presented below. These findings imply that the definition of the plateau modulus obtained from blob theory and classical rubber elasticity theory, $G_{N}^{0} \approx k_{B} T /\left(n_{e} \xi^{3}\right)$ with $\xi \approx R_{g}\left(c / c^{*}\right)^{v /(1-3 v)}$ can only be used up to a reduced concentration of about $c / c_{e}=2.0$ for our solutions, which corresponds roughly to polymer volume fractions higher than about $15 \%$.

This breakdown in de Gennes blob-theory scaling laws occurring at polymer volume fractions higher than about $15 \%$ can be ascribed to a transition from the semidilute to the concentrated regime. As explained by Milner, ${ }^{9}$ with increasing concentration, the correlation blob size $\xi$ shrinks, eventually down to the size of the "thermal blob" $l_{s}$, below which the conformation of polymer chain follows random-walk scaling even in a good solvent, as shown in Figure 1 of Hayward and Graessley. ${ }^{34}$ The thermal blob size is 
the length scale at the energy associated with excluded volume interactions reaches the thermal energy, which for reasons of entropy maximization favors the random walk. Milner called this length scale "swelling length" because the excluded volume swelling effect sets in above this length. The swelling volume fraction $\phi_{s}$ at which the blob size equals the swelling length, is estimated using $\phi_{s}=N_{s} \Omega_{0} / l_{s}^{3}$, where $N_{s}$ is the number of monomers in a chain of thermal blob size $l_{s}$, and $\Omega_{0}$ is the volume of a monomer. One can simplify this using the "packing length" $l_{p}$, which depends only on polymer stiffness and bulkiness, and is independent of solvent; it is defined as $l_{p}=N \Omega_{0} / R^{2}(N)$. Since $R^{2}\left(N_{s}\right)=l_{s}^{2}=N_{s} b^{2}$, we have $l_{p}=N_{s} \Omega_{0} /\left(N_{s} b^{2}\right)$, and in turn, we obtain

$$
\phi_{s}=l_{p} / l_{s} .
$$

Note that the weaker the solvent (i.e., the closer it is to being a theta solvent), the larger the swelling length is, and the smaller is the swelling concentration. However, since Eq. (13) is a scaling relationship with an unknown prefactor of order unity, ${ }^{9}$ we cannot calculate directly the swelling concentration for PS/TCP. Nevertheless, we note that Milner's calculated value of $\phi_{s}=0.24$ for polystyrene-benzene is not too far from the volume fraction (around 0.15) at which we see a breakdown in superposition based on the correlation blob, and we expect the swelling concentration for our system, PS/TCP, to be smaller than for PS/benzene, since benzene is a better solvent for PS than is TCP. Milner also nicely summarized the proposed concentration scaling relationships for the tube diameter $a(\phi)$, the number of monomers per entanglement $N_{e}(\phi)$, and the plateau modulus $G_{N}^{0}(\phi)$, above and below $\phi_{s}$, respectively based on Colby-Rubinstein conjecture for $\Theta$ solutions, and de Gennes’ blob model for polymer solutions in a good solvent, with 
the prefactors chosen to match the scaling relationships at the crossover point. (We will give Milner's scaling relationships in the next section.) For our PS/TCP solutions, we do not have enough data above the swelling concentration to test the Colby-Rubinstein scaling behavior. However, other data in the literature allows us to check this scaling behavior above $\phi_{s}$, and so we examine these data in the next section.

\subsubsection{Melt vs. Solution Rheology}

As alluded to in previous section, Milner ${ }^{9}$ proposed the following crossover between scaling relationships for the tube diameter $a(\phi)$ for concentrated solutions $\left(\phi_{s}<\phi \leq 1\right)$ for which Colby-Rubinstein scaling applies, and semidilute entangled solutions $\left(\phi_{e}<\phi<\phi_{s}\right)$, for which de Gennes blob scaling applies, with $\phi_{e}$ the volume fraction corresponding to $c_{e}$,

$$
a(\phi)=\left\{\begin{array}{l}
a(1) \phi^{-\alpha_{c} / 2}, \quad \phi>\phi_{s} \\
a(1) \phi_{s}^{-\alpha / 2}\left(\phi / \phi_{s}\right)^{v /(1-3 v)}, \quad \phi<\phi_{s}
\end{array} .\right.
$$

Note that in the semidilute regime, this scaling law for $a(\phi)$ is the same as the de Gennes scaling law for the blob size $\xi(\phi)$. Since for entangled solutions in the semidilute regime, $G_{N}^{0} \approx k_{B} T /\left(a^{2} \xi\right)$, while the de Gennes scaling is $G_{N}^{0} \approx k_{B} T / \xi^{3}$, these two scaling laws are identical.

The exponent $\alpha_{c}$ should take on the value, $\alpha_{c}=4 / 3=1.33$ in $\Theta$ solvents, according to the Colby-Rubinstein conjecture. As Milner notes, however, above the swelling concentration $\phi_{s}$, in any solvent, the polymer configuration is a random walk on all length scales, and hence the Colby-Rubinstein $\Theta$ solvent scaling applies for all polymer- 
solvent systems above $\phi_{s}$, and the dilution exponent $\alpha$ should therefore take on the universal value $\alpha_{c}$ above $\phi_{s}$. Nevertheless, in what we use $\alpha_{c}$ as a slightly polymersolvent-system specific parameter since many experiments have shown values of $\alpha_{c}$ that differ slightly (by a few percent at most) from 1.33 .

We obtain the number of monomers per entanglement at a volume fraction $\phi$ from Milner, ${ }^{9}$

$$
N_{e}(\phi)=\left\{\begin{array}{l}
N_{e}(1) \phi^{-\alpha_{c}}, \quad \phi>\phi_{s} \\
N_{e}(1) \phi_{s}^{-\alpha_{c}}\left(\phi / \phi_{s}\right)^{1 /(1-3 v)}, \quad \phi<\phi_{s}
\end{array} .\right.
$$

Here, $\quad N_{e}(\phi)=M_{e}(\phi) / M_{0}$ with $M_{0}$ the monomer molecular weight, and $M_{e}(\phi)=(4 / 5) \rho \phi R T / G_{N}^{0}(\phi)$. Thus, the modulus scales as $G_{N}{ }^{0} \propto \phi / N_{e} \propto \phi^{1+\alpha}$. In the concentrated region, $\phi>\phi_{s}, \alpha=\alpha_{c} \approx 1.33$, while in the semidilute regime, $\phi<\phi_{s}$, we recover the de Gennes scaling where $\alpha=1 /(3 v-1)$, and $G_{N}{ }^{0} \propto \phi^{3 v /(3 v-1)}$. Since the correlation blob size $\xi$ is proportional to $\phi^{v /(1-3 v)}$ within the semidilute regime (below $\phi_{s}$ ), and the excluded volume effect due to solvent is screened out at any length scale above $l_{s}$ when the volume fraction goes above $\phi_{s}$, we have

$$
\xi(\phi)=\left\{\begin{array}{l}
b \phi^{-1}, \quad \phi>\phi_{s} \\
b \phi_{s}^{-1}\left(\phi / \phi_{s}\right)^{v /(1-3 v)}, \quad \phi<\phi_{s},
\end{array},\right.
$$

and

$$
g(\phi)=\phi \xi^{3} / b^{3}=\left\{\begin{array}{l}
\phi^{-2}, \quad \phi>\phi_{s} \\
\phi_{s}^{-2}\left(\phi / \phi_{s}\right)^{1 /(1-3 v)}, \quad \phi<\phi_{s}
\end{array} .\right.
$$

Finally, from Eqs. 15 and 17, we obtain a formula for the number of blobs per entanglement $N_{e}(\phi) / g(\phi)$ : 


$$
N_{e}(\phi) / g(\phi)=\left\{\begin{array}{ll}
N_{e}(1) \phi^{2-\alpha_{c}}, & \phi>\phi_{s} \\
N_{e}(1) \phi_{s}^{2-\alpha_{c}}, & \phi<\phi_{s}
\end{array} .\right.
$$

Note that the coefficients of the equations are chosen to satisfy continuity of the variables at $\phi=\phi_{s}$ and $\phi=1$. For example, at $\phi=1$, the correlation blob size is equal to the statistical segment length $b$ from $\left\langle R^{2}\right\rangle_{0}=N b^{2}=[N(1) / g(1)] \xi(1)^{2}$, and the number of monomers per blob $g$ is unity.

The above can be summarized as follows. For a very good solvent with $v=0.588$, the dilution exponent below the swelling concentration $\phi_{s}$ is $\alpha=1 /(3 v-1) \approx 1.31$, while above $\phi_{s}$, the exponent is almost the same, $\alpha=\alpha_{c}=1.33$. Thus, for a very good solvent, the dilution exponent is essentially constant across the whole concentration range, as observed experimentally. For a marginally good- solvent system, like PS/TCP (for which $v=0.53), \alpha$ is larger ( $\alpha \approx 1.7$ ), and $\alpha$ can approach $\alpha=2$ as the solvent quality approaches $\Theta$ quality. However, as the solvent quality worsens, the swelling concentration drops, and the range of concentration over which $\alpha$ can exceed $\alpha_{c}$ diminishes. When the $\Theta$ condition is reached, then the Colby-Rubinstein argument applies for essentially all concentrations above dilute and $\alpha=\alpha_{c}=1.33$. Thus, the dilution exponent exhibits a non-monotonic behavior: for both very good and $\Theta$ solvents, $\alpha$ is essentially constant at a "universal value" of around 1.3-1.33 over the entire concentration range, while for marginally good solvents, the exponent is higher than this at low polymer concentrations only. As illustrated in Figure 3.6, if we start at some intermediate solvent quality and worsen the quality, the magnitude of the deviation in the exponent at low concentrations from the "universal value" increases, but the range of concentrations over which this deviation occurs shrinks to zero as the solvent quality is 
worsened towards the theta condition. On the other hand, if we increase the solvent quality, the range of concentrations over which there is a deviation from the "universal exponent” increases, while the deviation itself shrinks nearly to zero when the solvent becomes very good. Thus, the same scaling behavior is reached at both end points ( $\Theta$ and very good solvent quality), but in different ways. This rather subtle, and non-intuitive, behavior has led to confusion in the literature regarding whether or not the dilution exponent has a universal value for all polymer/solvent pairs over all concentrations.

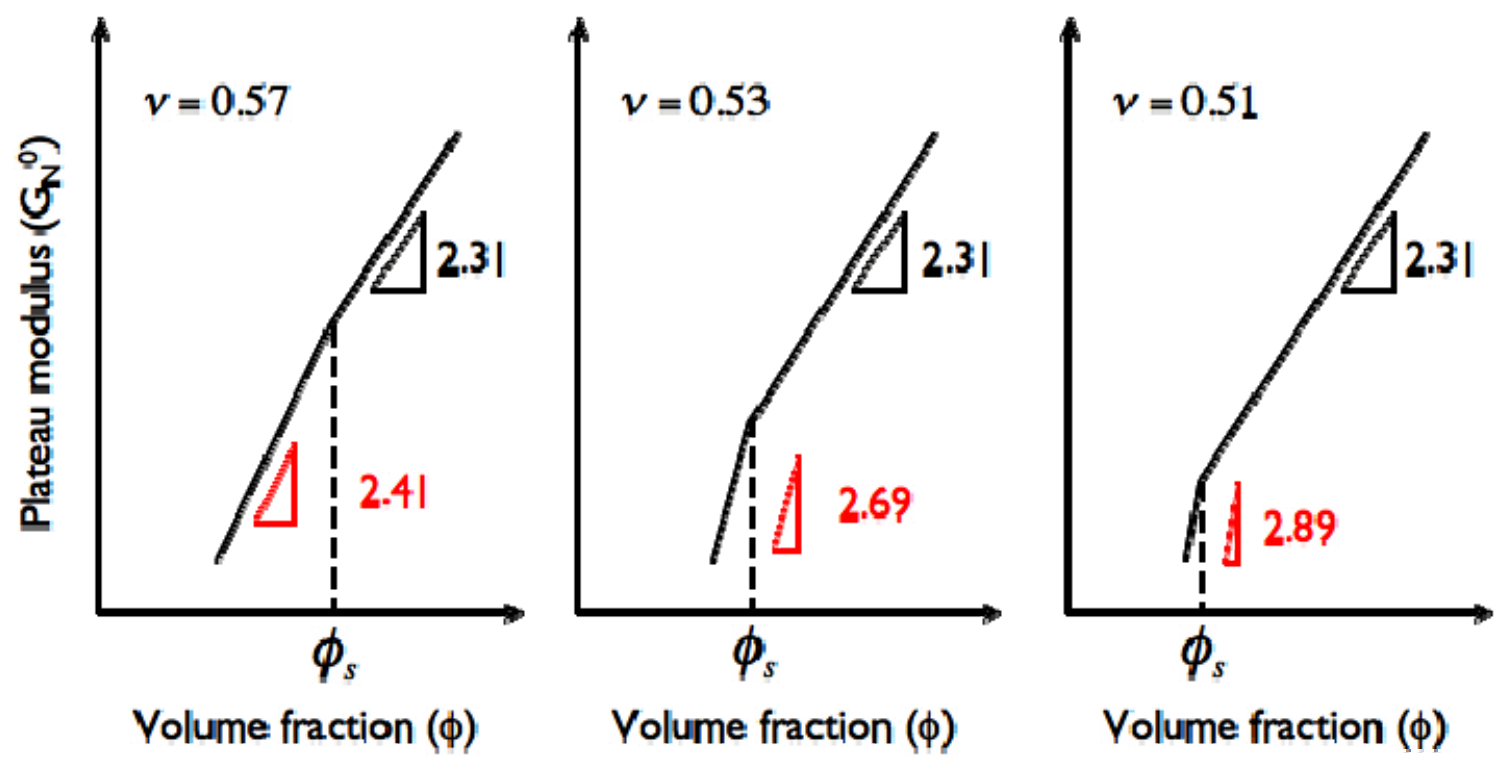

Figure 3.6. Schematic of the scaling of plateau modulus $G_{N}^{0}(\phi)$ of polymer solutions with three different excluded volume exponents, namely $v=0.57,0.53$, and 0.51 , versus volume fraction $\phi$. See text for detail.

\subsubsection{Nearly Monodisperse 1,4 Polybutadiene Melts and Solutions in Phenyl Octane}

In principle, once the reduced concentration $c / c_{e}$ exceeds unity, the solution is entangled, and the blob theory for a semidilute polymer solution or the Colby-Rubinstein 
scaling for a concentrated polymer solution implies that such a solution ought to be "dynamically equivalent" to a melt having the "same" entanglement density. For semidilute or concentrated entangled solutions, the degree of entanglement can be measured by the ratio $c / c_{e}$, while for melts, it is usually measured by the ratio, $M / M_{e}$ (or $N / N_{e}$ ) of the molecular weight, $M$ to the molecular weight between entanglements $M_{e}$. We will call this latter ratio the "entanglement density," and will show in what follows that it can also be defined for semidilute solutions, once the dilution exponent is determined. In addition to establishing a common measure of entanglement density for solutions and melts so that "dynamically equivalent" entangled solutions or melts can be identified, the frequency and modulus must be appropriately rescaled, so that data for both solutions and melts can be plotted using common reduced variables.

With this in mind, we now examine an extensive set of data for nearly monodisperse 1,4 polybutadiene (PBd) solutions in phenyl octane $(\mathrm{PhO})$ and melts of $\mathrm{PBd}$ of various molecular weights, published by Colby et al. ${ }^{5}$ To plot these data on a universal scale, we use the parameters for PBd melts that Likhtman and McLeish ${ }^{28}$ determined by fitting the data of PBd melts at $28^{\circ} \mathrm{C}$ of Baumgaertel et $a .^{35}$ with the Likhtman-McLeish (LM) theory. They thereby determined the values $G_{N}^{0}=1.47 \times 10^{6} \mathrm{~Pa}, M_{e}=1.93 \times 10^{3} \mathrm{~g} / \mathrm{mol}$ (note that $M_{e}$ was used as an independent fitting parameter, not calculated from $\left.M_{e}=(4 / 5) \rho R T / G_{N}^{0}\right)$, and $\tau_{e, D E}=4.9 \times 10^{-7} \mathrm{~s}$. Here, we have shifted slightly $\tau_{e, D E}$ at $28^{\circ} \mathrm{C}$ to the value $\tau_{e, D E}=5.66 \times 10^{-7} \mathrm{~s}$ at $25^{\circ} \mathrm{C}$ using the WLF equation with the parameters $C_{1}=3.48, C_{2}=163 \mathrm{~K}$, and $T_{0}=25^{\circ} \mathrm{C} .^{5}$ The plateau modulus of a $\mathrm{PBd} / \mathrm{phenyl}$ octane $(\mathrm{PhO})$ solution at $25^{\circ} \mathrm{C}$ scales as follows: 


$$
G_{N, P B d}^{0}(\phi)=G_{N, P B d}^{0}(1) \phi^{2.29} .
$$

This scaling relation was determined from experimental measurements of the plateau modulus of $\mathrm{PBd} / \mathrm{PhO}$ solutions against volume fraction. ${ }^{5}$

Because the glass transition temperature of this solution at any volume fraction is at least $100 \mathrm{~K}$ lower than the experimental temperature $298 \mathrm{~K}$ we can use the Fox equation, $1 / T_{g}(\phi)=\phi / T_{g, \text { polymer }}+(1-\phi) / T_{g, \text { solvent }}$. Here, the glass transition temperature of bulk PBd is $T_{g, P B d}=174 \mathrm{~K}$ and that of $\mathrm{PhO}$ is $T_{g, \text { PhO }}=152 \mathrm{~K}$. Thus the glass transition temperatures of the solutions are in the narrow range between $152 \mathrm{~K}$ and $174 \mathrm{~K}$, well below the temperatures at which the measurements were made $\left(25^{\circ} \mathrm{C}\right)$ and thus the polymer has a similar monomeric friction coefficient in all solutions and the melt. Colby et $a .^{5}{ }^{5}$ were able to obtain complex moduli of PBd/PhO solutions $\left(M_{w}=925,000 \mathrm{~g} / \mathrm{mol}, M_{w} / M_{n}<1.1\right.$, and $50 \%$ cis $1,4,42 \%$ trans 1,4 , and $8 \%$ vinyl) at various volume fractions from 0.03 up to unity. The swelling volume fraction $\phi_{s}$ for PBd-PhO solutions can be bounded from above by the value found for PBd in the good solvent cyclohexane, which is 0.045 . Note that PhO is a poorer solvent than cyclohexane for PBd ( $v=0.609$ for PBd/cyclohexane, ${ }^{9}$ while $v=0.554$ for $\mathrm{PBd} / \mathrm{PhO}^{5}$ ) implying that $\mathrm{PBd} / \mathrm{PhO}$ solution has a longer swelling length, and in turn smaller swelling volume fraction $\left(\phi_{s}=l_{p} / l_{s}\right)$, than does $\mathrm{PBd} /$ cyclohexane since the packing length is independent of solvent quality. From this, we estimate that $\phi_{s}$ for $\mathrm{PBd} / \mathrm{PhO}$ solutions should be significantly less than 0.045 . Therefore, we can safely treat all $\mathrm{PBd} / \mathrm{PhO}$ solutions of Colby et al. ${ }^{5}$ as concentrated solutions with $v=0.5$ and $\alpha=1.29$. This is a remarkable fact for polybutadiene solutions. Even though many researchers ${ }^{7,36,37}$ have demonstrated that the dilution exponent of PBd solutions in marginally good to good solvents is independent of solvent 
quality in seeming contradiction to de Gennes' scaling theory, it turns out that this is not because there is no difference in dilution exponent between good solvent and $\Theta$ solvent within the semidilute regime, but instead is because the volume fractions of almost all PBd solutions studied actually belong to the concentrated regime, not the semidilute regime. For polystyrene solutions, where $\phi_{s}$ is much larger (around 0.15-0.25), the volume fractions used in our studies presumably cover both semidilute and concentrated regimes. Consistent with this, we showed in the last section that the de Gennes' scaling law collapses our PS/TCP data in the semidilute regime, where we used a dilution exponent of $\alpha=1.7$, based on the excluded volume exponent, rather than the exponent near 1.3, while superposition based on the exponent $\alpha=1.7$ gradually breaks down with increasing volume fraction.

To relate $\tau_{e, \text { scaling }}$ with $\tau_{e, D E}$ at a volume fraction that lies in concentrated regime, we take advantage of the fact that Colby et al. ${ }^{5}$ obtained both PBd melt and solution data at the same temperature of $25^{\circ} \mathrm{C}$. Thus, we can match the formula for $\tau_{e, D E}$ for a PBd melt to that for a PBd solution simply by setting the "solution" volume fraction to $\phi=1$, and requiring the two formulas to give the same answer. We use the value of $\tau_{e, D E}$ of a $\mathrm{PBd}$ melt that Likhtman and McLeish obtained by fitting their model (the Likhtman-McLeish (LM) model) to the experimental melt data of Baumgaertel et al. ${ }^{36}$ by LM model, ${ }^{28}$ giving $\tau_{e, D E}=5.66 \times 10^{-7} \mathrm{~s}$. Now we can obtain $\tau_{e, \text { scaling }}(\phi)$ for a PBd solution with the volume fraction ranging $\phi_{s}<\phi \leq 1$ from Eqs. (11), (16) and (18),

$$
\tau_{e, \text { scaling }}=\frac{\eta_{s}}{k_{B} T} \xi^{3}\left[N_{e}(\phi) / g(\phi)\right]^{2}=\frac{\eta_{s}}{k_{B} T} b^{3} N_{e}^{2}(1) \phi^{1-2 \alpha_{c}}
$$


Comparing $\tau_{e, D E}=5.66 \times 10^{-7} \mathrm{~s}$ and $\tau_{e, \text { scaling }}(1)=8.68 \times 10^{-8} \mathrm{~s}$ thus gives us the prefactor, $K_{1}=6.52$ in $\tau_{e, D E}=K_{1} \cdot \tau_{e, \text { scaling }}$ for this particular polymer-solvent pair without adjustable parameters. Since we have a series of $G^{\prime}$ and $G^{\prime \prime}$ curves for PBd melts and solutions available, we are now able to compare these functions for varying $M / M_{e}$ for melts and solutions, plotted as: $G^{\prime} / G_{N}^{0} \& G^{\prime \prime} / G_{N}^{0}$ versus $\omega \tau_{e, D E} \cdot \tau_{e, D E}(\phi)=K_{1} \cdot \tau_{e, \text { scaling }}(\phi)$ and $G_{N}^{0}(\phi)$ are tabulated in Table 3.5, and the plots are shown in Figure 3.7, with filled symbols representing solutions and open symbols melts.

Table 3.5. The equilibration times and the plateau moduli of a series of $\mathrm{PBd} / \mathrm{PhO}$ solutions $\left(M_{w}=9.25 \times 10^{5} \mathrm{~g} / \mathrm{mol}\right)$. Here, we employ $N_{e}(\phi)=N_{e}(1) \phi^{-\alpha_{c}}$ to compute the number of monomers per entanglement at a volume fraction $\phi$.

\begin{tabular}{|c|c|c|c|}
\hline$\phi$ & $N / N_{e}(\phi)$ & $\begin{array}{c}K_{1} \cdot \tau_{e, \text { scaling }}(\phi) \\
\mathrm{s}\end{array}$ & $\begin{array}{c}G_{N}^{0}(\phi) \\
\mathrm{Pa}\end{array}$ \\
\hline 0.021 & 3.4 & $7.03 \times 10^{-4}$ & $2.58 \times 10^{2}$ \\
\hline 0.027 & 4.6 & $3.82 \times 10^{-4}$ & $4.50 \times 10^{2}$ \\
\hline 0.062 & 13.3 & $5.19 \times 10^{-5}$ & $2.83 \times 10^{3}$ \\
\hline 0.140 & 37.9 & $7.88 \times 10^{-6}$ & $1.76 \times 10^{4}$ \\
\hline 0.280 & 92.8 & $1.82 \times 10^{-6}$ & $8.39 \times 10^{4}$ \\
\hline 0.488 & 184.1 & $7.15 \times 10^{-7}$ & $2.93 \times 10^{5}$ \\
\hline 1.000 & 479.3 & $5.66 \times 10^{-7}$ & $1.47 \times 10^{6}$ \\
\hline
\end{tabular}



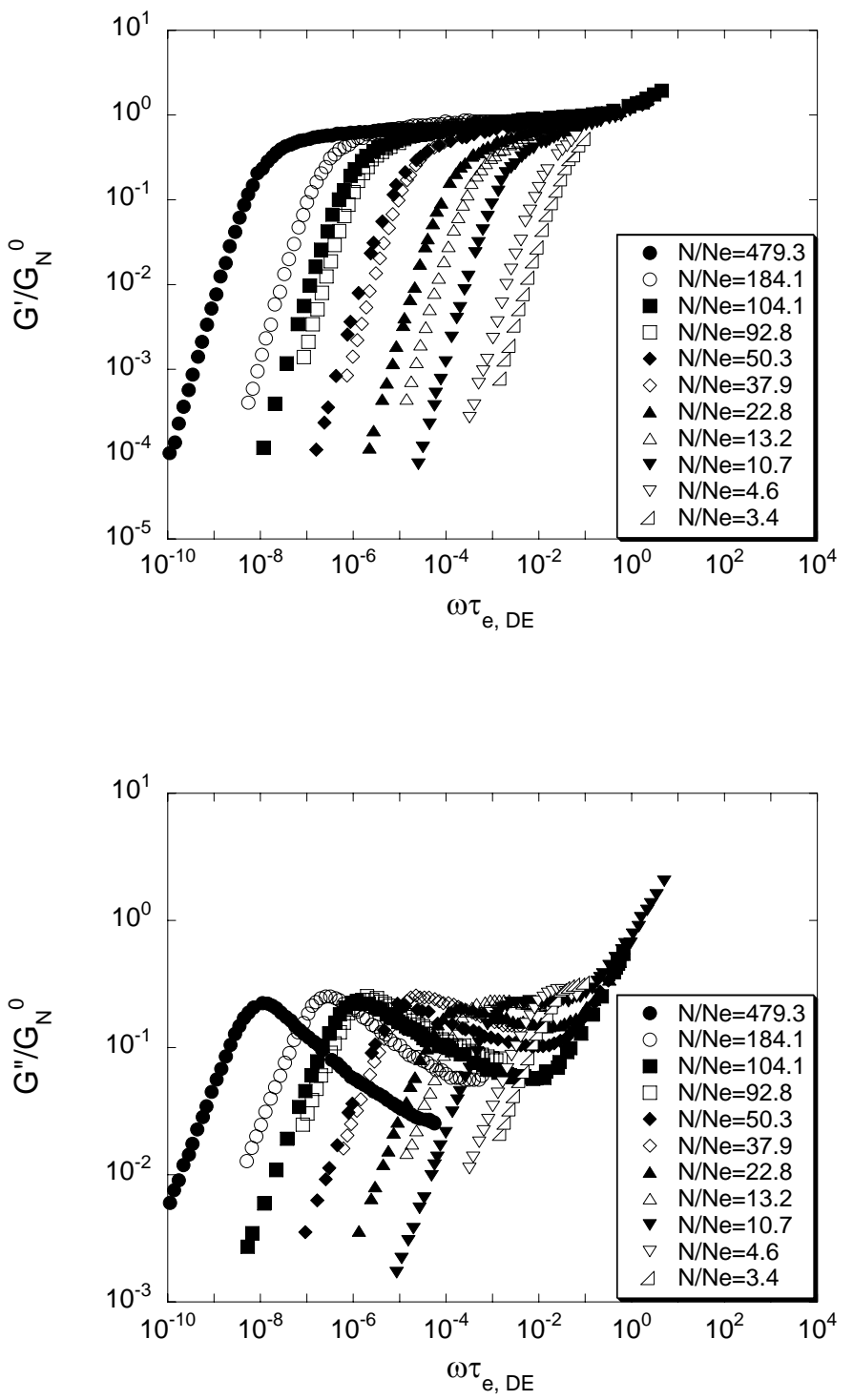

Figure 3.7. Normalized complex moduli, $G^{\prime} / G_{N}^{0}$ and $G^{\prime \prime} / G_{N}^{0}$ against normalized frequency, $\omega \tau_{e, D E}$, for nearly monodisperse polybutadiene (PBd) melts and solutions. Here, $\tau_{e, D E}$ for PBd solutions is computed from $\tau_{e, D E}=K_{1} \cdot \tau_{e, \text { scaling }}, K_{1}=6.52$, and Eq. (20), with $\alpha_{c}=1.29$, and $N_{e}(1)=35.7$ (see Table 3.5). Filled symbols denote $G^{\prime} / G_{N}^{0}$ and $G^{\prime \prime} / G_{N}^{0}$ for PBd melts with $M_{w}=9.25 \times 10^{5}, 2.01 \times 10^{5}, 0.97 \times 10^{5}, 0.44 \times 10^{5}$, and $0.21 \times 10^{5}$ $\mathrm{g} / \mathrm{mol}^{36}$ and open symbols denote PBd solutions with $M_{w}=9.25 \times 10^{5} \mathrm{~g} / \mathrm{mol}$ at volume fractions of $0.488,0.280,0.140,0.062,0.027$, and $0.021 .^{5}$ 
Note in Figure 3.7 that treating all solutions and melts as "concentrated solutions" puts all data (melts and solutions) into a simple progression. As $N / N_{e}(\phi)$ increases, either because of increased molecular weight of increased concentration (and therefore lower $N_{e}(\phi)$ ), the transition to terminal behavior occurs at a lower reduced frequency. Note also that a melt and a solution with almost the same value of $N / N_{e}(\phi)$ (open and filled squares) have almost identical $G^{\prime}$ and $G^{\prime \prime}$ curves. Unlike PS/TCP solutions, for PBd/PhO solutions a single scaling relationship for the dependence of the plateau modulus on $\phi$, $G_{N}^{0}(\phi)=G_{N}^{0}(1) \phi^{2.29}$, is observed to hold at volume fractions from $100 \%$ down to $3 \%{ }^{5}$ Note that the loss moduli of $\mathrm{PBd} / \mathrm{PhO}$ solutions show a slight indication of an "early" upturn at high frequencies, which is not present in the melt. If real, and if the "early" upturn continues at higher frequencies, then there may be a significant, and unexplained difference between solutions and melts at high frequency.

From the data of Figure 3.7, we can extract a reduced crossover frequency, $\omega_{X} \tau_{e, D E}$ by extrapolating the data for $G^{\prime} / G_{N}^{0}$ and $G^{\prime \prime} / G_{N}^{0}$ in the terminal region where $G^{\prime} \propto \omega$ and $G^{\prime \prime} \propto \omega^{2}$ up to the reduced frequency where $G^{\prime} / G_{N}^{0}$ crosses $G^{\prime \prime} / G_{N}^{0}$. From the crossover frequencies of a series of PBd solutions, we get a scaling relationship between the reduced crossover relaxation time, $\tau_{X} / \tau_{e, D E}=1 /\left(\omega_{X} \tau_{e, D E}\right)$ and $N / N_{e}$; see Figure 3.8. 


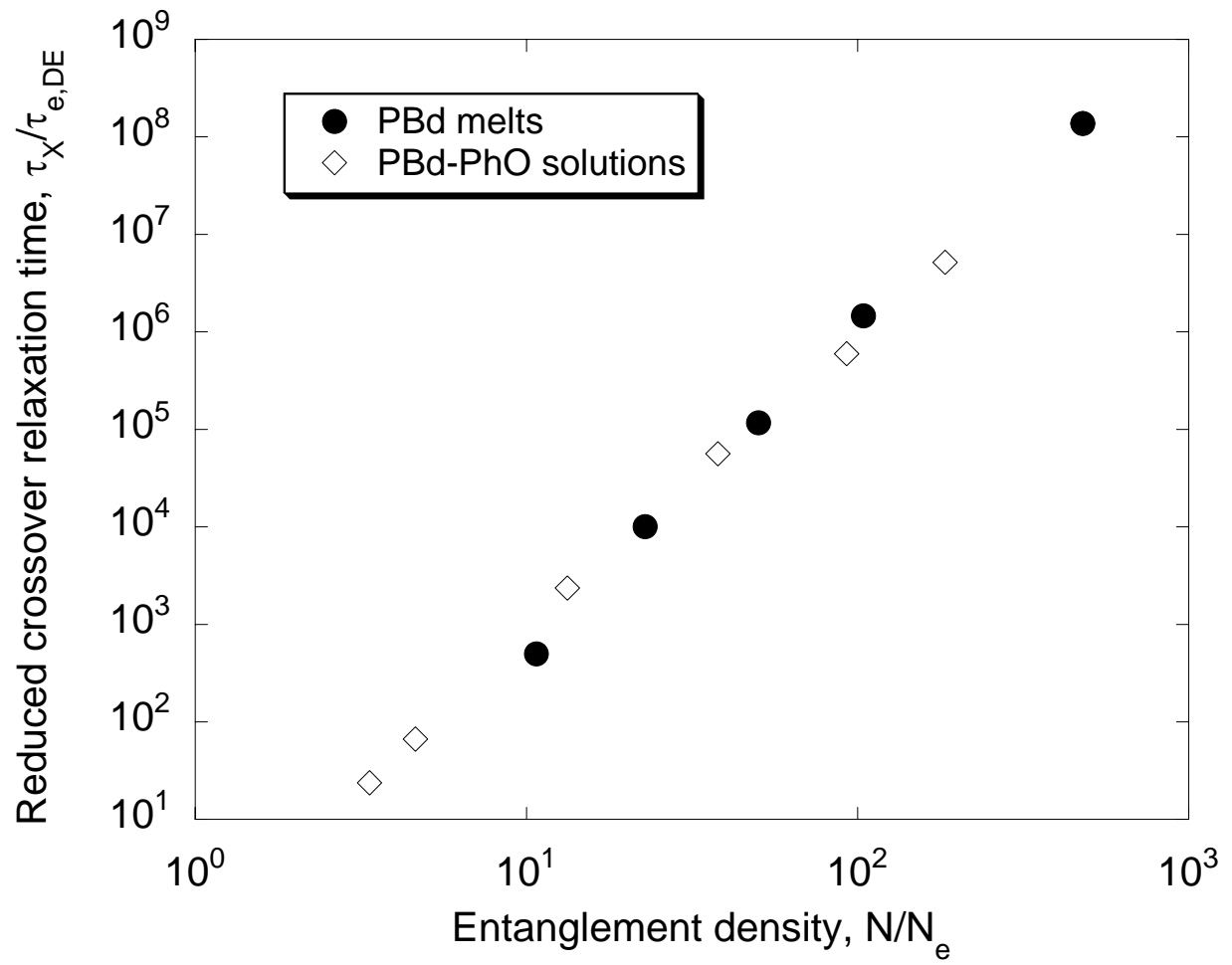

Figure 3.8. The reduced crossover relaxation time of polybutadiene (PBd) melts and $\mathrm{PBd} /$ phenyl octane $(\mathrm{PhO})$ solutions versus the number of entanglements per molecule: $N / N_{e}$ for melts and solutions. Here, we employ $M_{e}(1)=1.93 \times 10^{3} \mathrm{~g} / \mathrm{mol}$ from Likhtman and McLeish ${ }^{28}$ to obtain $N_{e}(1)=M_{e}(1) / M_{0}$ with $M_{0}=54 \mathrm{~g} / \mathrm{mol}$ the monomer molecular weight of PBd. For solutions, we use the scaling law $N_{e}(\phi)=N_{e}(1) \phi^{-\alpha_{c}}=\left(1.93 \times 10^{3} / 54\right) \phi^{-}$ 1.29. Filled circles represent PBd melts $\left(N / N_{e}=10.7,22.9,50.3,104.2\right.$, and 479.3) and open diamonds represent $\mathrm{PBd} / \mathrm{PhO}$ solutions of $M_{\mathrm{w}}=9.25 \times 10^{5}\left(N / N_{e}=3.4,4.6,13.3\right.$, 37.9, 92.8, 184.1, and 479.3).

Figure 3.8 shows us the similarities between melts and solutions. The best-fit powerlaw slope of the line for melts is $3.27 \pm 0.09$ and that for solutions is $3.09 \pm 0.05$, which suggests that, almost to within experimental error, an entangled concentrated solution is essentially the same as a "melt of correlation blobs." 


\subsubsection{Extension to Binary Blends}

Most synthetic polymers have broad molecular weight distributions and some polydispersity is inevitable even when synthesizing nearly monodisperse linear polymers through anionic polymerization. Since polydisperse systems are both more common and more relevant commercially, we would like to encompass polydispersity within the “universal scaling” law.

Binary blends of monodisperse polymers provide a first step to confirm the validity of the "blob" model scaling for polydisperse polymer solutions. Comparing both linear and nonlinear rheological responses of one binary blend with another having the same ratio of short- to long-chain molecular weight and the same $c / c_{e}$ should determine the validity of the universal scaling for polydisperse semidilute solutions that are not monodisperse. Here, we base $c / c_{e}$ on the weight average molecular weights of the short and long chains, each of which occupies 50 weight $\%$ of the polymer; see Table 3.6 .

Table 3.6. Weight-average molecular weights and parameters of two binary blends of PS samples in TCP

\begin{tabular}{|c|c|c|c|c|c|c|c|c|c|}
\hline & \multicolumn{3}{|c|}{ Molecular weights and ratios } & \multicolumn{4}{c|}{ Parameters of short chain polymer } \\
\hline & $\begin{array}{c}M_{w, \text { short }} \\
\mathrm{g} / \mathrm{mol}\end{array}$ & $\begin{array}{c}M_{w, \text { long }} \\
\mathrm{g} / \mathrm{mol}\end{array}$ & $\begin{array}{c}M_{w, \text { long }} / \\
M_{w, \text { short }}\end{array}$ & $v^{\mathrm{a}}$ & $\begin{array}{c}{[\eta]_{0}^{\mathrm{a}}} \\
\mathrm{L} / \mathrm{g}\end{array}$ & $\begin{array}{c}R_{g}{ }^{\mathrm{b}} \\
\mathrm{nm}\end{array}$ & $\begin{array}{c}c^{{ }^{*} \mathrm{c}} \\
\mathrm{g} / \mathrm{L}\end{array}$ & $\begin{array}{c}M_{w} A_{2}{ }^{\mathrm{d}} \\
\mathrm{L} / \mathrm{g}\end{array}$ & $\begin{array}{c}C_{e}{ }^{\mathrm{e}} \\
\mathrm{g} / \mathrm{L}\end{array}$ \\
\hline Blend 1 & $1.28 \times 10^{6}$ & $2.68 \times 10^{6}$ & 2.09 & 0.53 & 0.17 & 43.87 & 25.19 & 0.26 & 84.32 \\
\hline Blend 2 & $2.68 \times 10^{6}$ & $5.56 \times 10^{6}$ & 2.07 & 0.53 & 0.26 & 64.90 & 16.29 & 0.40 & 54.52 \\
\hline
\end{tabular}


${ }^{\mathrm{b}} R_{v} / R_{g}=0.74$ for linear PS solutions over the range $10^{5} \leq M_{w} \leq 10^{6} \cdot{ }^{31}$ Here, the viscometric radius of the polymer chain $R_{v}$ is calculated as $R_{v}=\left(\frac{3[\eta]_{0} M_{w}}{10 \pi N_{A}}\right)^{1 / 3}$ with $N_{A}$ Avogadro's number, and $R_{g}=\left(\frac{3[\eta]_{0} M_{w}}{10 \pi N_{A}}\right)^{1 / 3} / 0.74^{3}$

${ }^{\mathrm{c}} C^{*} \equiv \frac{M_{\mathrm{w}}}{N_{A} R_{g}^{3}}$

${ }^{\mathrm{d}} M_{\mathrm{w}} A_{2}=6.5 / \mathrm{c}^{*}$ for PS in good solvents ${ }^{2}$

${ }^{\mathrm{e}} C_{e} \equiv\left(1 / M_{w} A_{2}\right) n_{e}^{3 v-1} \cdot 2,3$

As illustrated in Figures 3.9 and 3.10, two binary blends with almost the same ratio of short to long chain molecular weight and the same $c / c_{e}$ show excellent agreement in both linear and nonlinear rheological responses with exceptions for $c / c_{e}=2.50$ and 3.00, just as was the case for monodisperse PS/TCP solutions. This allows us to further extend the universal scaling from blob theory to polydisperse polymer solutions.

(a)

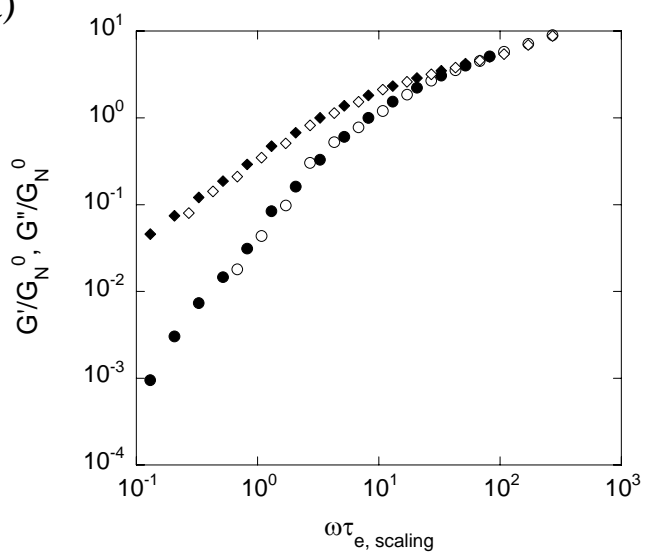

(b)

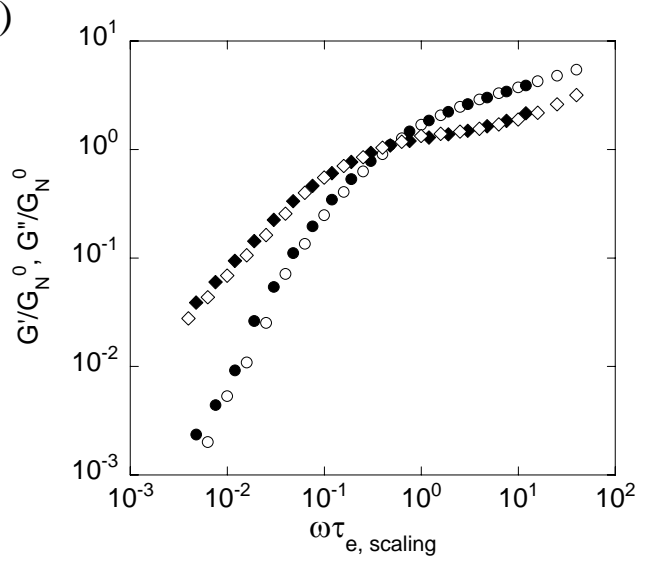


(c)

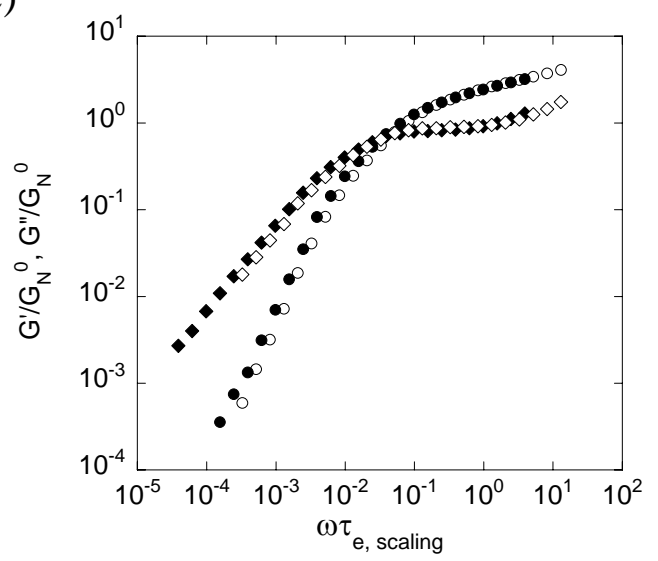

(e)

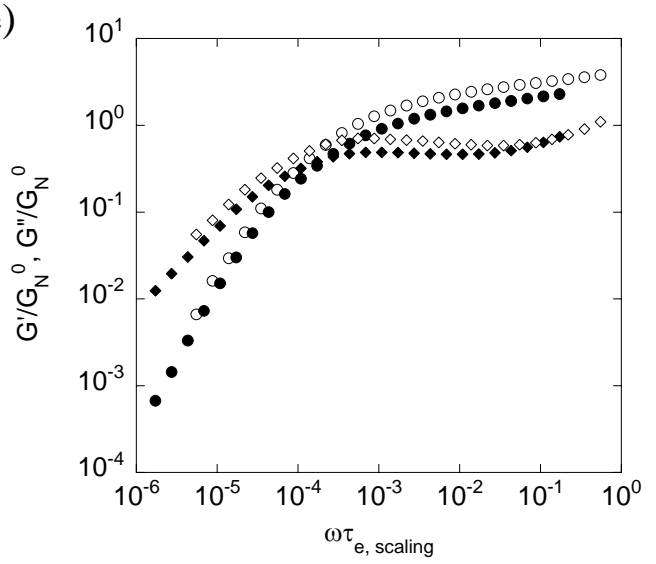

(d)

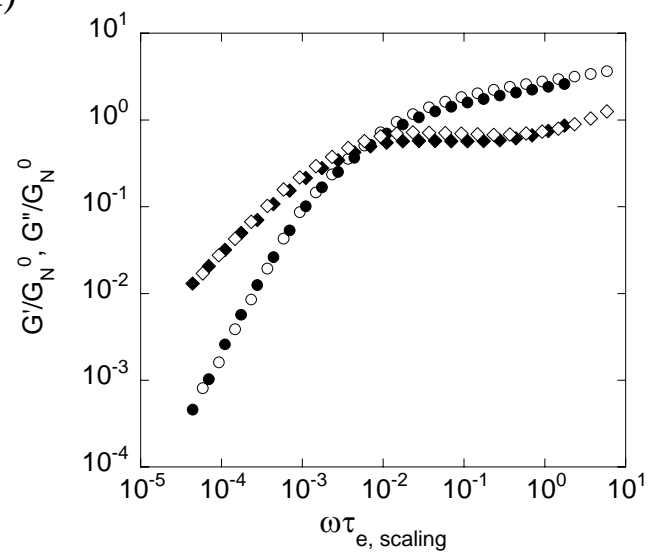

(f)

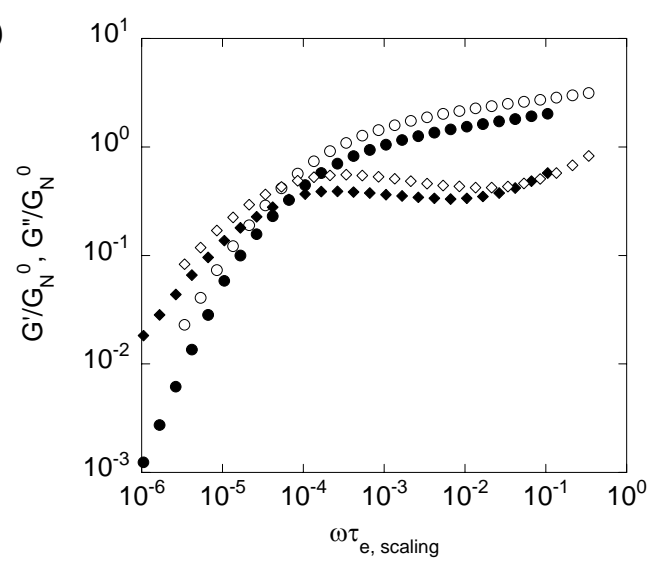

Figure 3.9. Superposition of linear viscoelastic properties of two binary blends of $\mathrm{PS} / \mathrm{TCP}$ solutions at six different values of $c / c_{e}$ : (a) $c / c_{e}=0.50$, (b) $c / c_{e}=1.00$, (c) $c / c_{e}=1.50$, (d) $c / c_{e}=2.00$, (e) $c / c_{e}=2.50$, and (f) $c / c_{e}=3.00$. Filled circles and diamonds respectively represent the normalized storage and loss moduli of the solutions with equal mass fractions of $M_{w, \text { short }}=1.28 \times 10^{6} \mathrm{~g} / \mathrm{mol}$ and $M_{w, \text { long }}=2.68 \times 10^{6} \mathrm{~g} / \mathrm{mol}$. Open circles and diamonds respectively represent the normalized storage and loss moduli of the solutions with $M_{w, \text { short }}=2.68 \times 10^{6} \mathrm{~g} / \mathrm{mol}$ and $M_{w, \text { long }}=5.56 \times 10^{6} \mathrm{~g} / \mathrm{mol} . \quad \tau_{e, \text { scaling }}$ is based on the weight average molecular weight of long and short chains. 


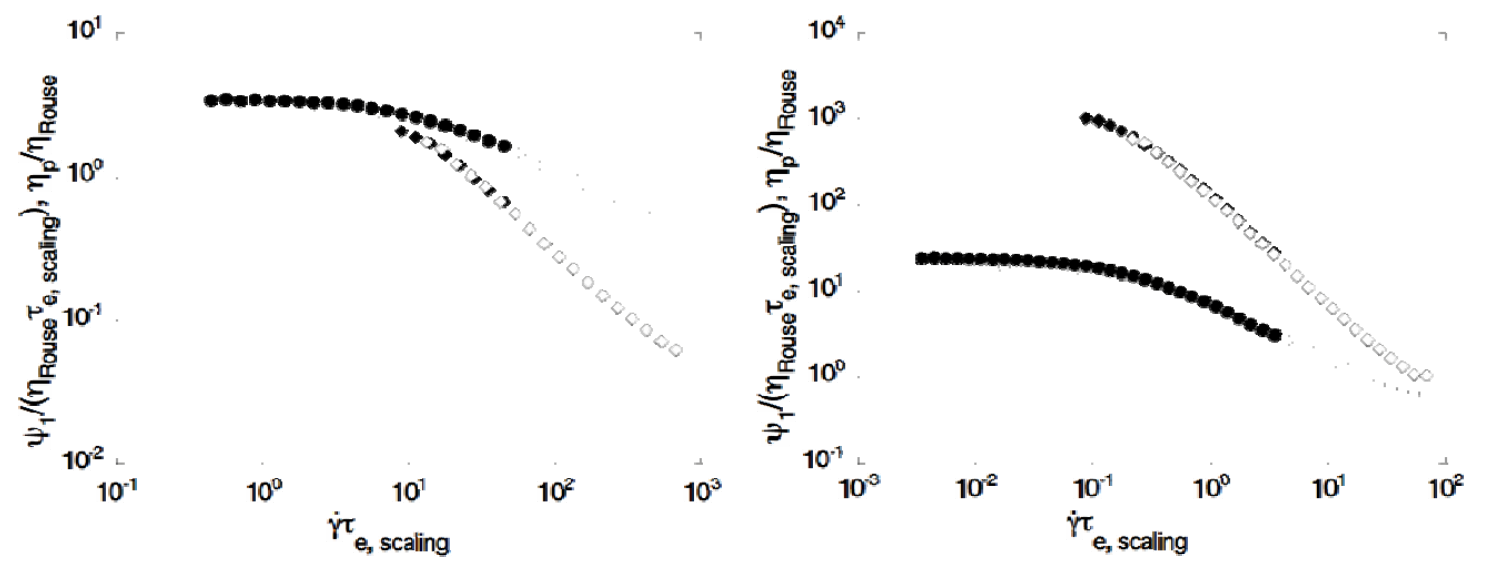

Figure 3.10. The same as Figure 3.9 except for nonlinear viscoelastic properties: (a) $c / c_{e}=0.50$, and (b) $c / c_{e}=1.00$.

\subsection{Conclusions}

We have explored the "universal scaling” of rheological properties of semidilute linear polymer solutions based on the "blob" model for semidilute solutions and based on the conjecture of Colby-Rubinstein for concentrated solutions. ${ }^{12}$ Starting with nearly monodisperse polystyrene solutions where "universal scaling" has already been confirmed for the zero-shear viscosity, ${ }^{3}$ we have tested the dynamic similarity of the frequency-dependent linear viscolelastic properties of polystyrene solutions, polybutadiene solutions and melts, and of binary blends of two monodisperse polystyrene solutions, as well as the shear-rate-dependent nonlinear properties of polystyrene solutions. We found that for polystyrene solutions in TCP, "universal scaling” using the de Gennes semidilute blob concept, which gives $G_{N}^{0}(\phi)=G_{N}^{0}(1) \phi^{3 v /(3 v-1)}$ and $\tau_{e, \text { scaling }}=\left(\eta_{s} / k_{B} T\right) R_{g}^{3}\left(c / c^{*}\right)^{3 v /(1-3 v)} n_{e}^{2}$ as scaling parameters, allows us to collapse all linear and nonlinear data, for both monodisperse and bidisperse solutions, at 
concentrations in the semidilute range, where the semidilute range for this polymer/solvent pair lies below a volume fraction of around 0.15 , which is roughly consistent with our estimated value of the "swelling concentration" $\phi_{s}$ identified by Milner. This swelling concentration is the concentration at which the size of a correlation blob at a volume fraction $\xi(\phi)$, equals that of thermal blob $l_{s}$. For polymer chains at volume fractions below $\phi_{s}$ there is a range of length scales over which the polymer is swollen in a good solvent, while above $\phi_{s}$ they follow random walk configurations at any length scale. At higher concentrations of PS in TCP, near the value we estimate for $\phi_{s}$, the semidilute scaling laws based on the above formulas fail to collapse the PS/TCP data. For $\phi>\phi_{s}$, we can extract the expressions for two normalizing parameters for universal scaling using the Colby-Rubinstein conjecture for $\Theta$ solutions): $G_{N}^{0}(\phi)=G_{N}^{0}(1) \phi^{1+\alpha_{c}}$ and $\tau_{e \text {, scaling }}=\left(\eta_{s} / k_{B} T\right) b^{3} N_{e}^{2}(1) \phi^{1-2 \alpha_{c}}$, where $\alpha_{c}$ is around 1.3 for any solvent quality. While we do not have enough data for PS/TCP at concentrations above $\phi_{s}$ to test these scaling laws, we were able to use data from Colby et al. ${ }^{5}$ for polybutadiene in phenyl octane to test and confirm these relationships for the concentrated regime over a wide range of concentrations all the way up to the melt, since the value of $\phi_{s}$ for these polybutadiene solutions appears to be very low. Thus, scaling theory for polymer solutions is confirmed for all viscoelastic data we have examined, as long as we recognize the crossover from semidilute concentrations, where the de Gennes blob theory holds, to the concentrated regime where the Colby-Rubinstein exponent seems to be valid. The melt state is thus viewed as just a special case of “concentrated solutions," and there is no behavior we could identify that is distinctive to solutions, except possibly at the very highest frequencies, where solutions seem to show an early "upturn” in G", before 
such an upturn is seen in the melts. Near the crossover between semidilute and concentrated solutions, no simple scaling is likely to apply, although if the crossover concentration were known precisely, it might even be possible to superimpose data from above the crossover onto the data below it, by using prefactors Milner obtained by imposing continuity on the scaling quantities at the crossover concentration $\phi_{s}$. 


\section{References}

1. de Gennes, P. -G. Scaling Concepts in Polymer Physics; Cornell University Press: Ithaca, New York, 1979.

2. Raspaud, E.; Lairez, D.; Adam, M. Macromolecules 1995, 28, 927-933.

3. Heo, Y.; Larson, R. G. J. Rheology 2005, 49, 1117-1128.

4. Solomon, M. J.; Muller, S. J. J. Polym. Sci., Polym. Phys. 1996, 334, 181-192.

5. Colby, R. H.; Fetter, L. J.; Funk, W. G.; Graessley, W. W. Macromolecules 1991, 24, 3873-3882.

6. Edwards, S. F. Proc. Phys. Soc. 1966, 88, 265-280.

7. Graessley, W. W. Polymeric Liquids \& Networks: Dynamics and Rheology; Garland Science: New York, 2008 (to be published).

8. Rubinstein, M.; Colby, R. H. Polymer Physics, Oxford University Press Inc., New York, 2003.

9. Milner, S. T. Macromolecules 2005, 38, 4929-4939.

10. Marin, G.; Montfort, J. P.; Monge, P. Rheol. Acta. 1982, 21, 4-5, 449-451.

11. Colby, R. H.; Rubinstein, M.; Daoud, M. Journal de Physique II (France) 1994, 4, 1299-1310.

12. Colby, R. H.; Rubinstein, M. Macromolecules 1990, 23, 2753-2757. 
13. Adam, M.; Delsanti, M. Journal de Physique (Paris) 1983, 44, 1185-1193.

14. Adam, M.; Delsanti, M. Journal de Physique (Paris) 1984, 45, 1513-1521.

15. Adam, M.; Lairez, D.; Raspaud, E. Journal de Physique II 1992, 2, 2067-2073.

16. Heo, Y; Larson, R. G. J. Rheol. 2007, 51, 1099-1100.

17. Doi, M.; Edwards, S. F. The Theory of Polymer Dynamics; Oxford University Press Inc.: New York, 1986.

18. Ferry, J. D. Viscoelastic Properties of Polymers, 3rd ed.; Wiley: New York, 1980.

19. Zimm, B. H. J. Chem. Phys. 1956, 24, 269-278.

20. Rouse, P. E. J. Chem. Phys. 1953, 21, 1272-1280.

21. Stepanek, P.; Perzynski, R; Delsanti, M.; Adam, M. Macromolecules 1984, 17, $2340-$ 2343.

22. Noda, I.; Kato, N.; Kitano, T.; Nagasawa, M. Macromolecules 1981, 14, 668-676.

23. Isono, Y.; Fujimoto, T.; Takeno, N.; Kaijiura, H.; Nagasawa, M. Macromolecules 1978, 11, 888-893.

24. Plazek, D. J.; Riande, E.; Markovitz, H.; Taghupathi N. J. Polym. Sci.: Polym. Phys. Ed. 1979, 17, 2189-2213.

25. Osaki, K.; Nishimura, Y.; Kurata, M. Macromolecules 1985, 18, 1153-1157. 
26. Osaki, K.; Takatori, E.; Tsunashima, Y.; Kurata, M. Macromolecules 1987, 20, 525529.

27. Fetters, L. J.; Lohse, D. J.; Richiter, T. A.; Zirkel, A. Macromolecules 1994, 27, 4639-4647.

28. Likhtman, A. E.; McLeish, T. C. B. Macromolecules 2002, 35, 6332-6343.

29. Larson, R. G.; Sridhar, T.; Leal, L. G.; McKinley, G. H.; Likhtman, A. E.; McLeish, T. C. B. J. Rheol. 2003, 47, 809-818.

30. Schausberger, A.; Schindlauer, G.; Janeschitzkriegl, H. Rheol. Acta. 1985, 24, $220-$ 227.

31. Graessley, W. W. Polymeric Liquids \& Networks: Structure and Properties; Garland Science: New York, 2004.

32. Larson, R. G. Rheol. Acta. 1992, 31, 213-263.

33. Pattamaprom, C.; Larson, R.G. Macromolecules 2001, 34, 5229-5237.

34. Hayward, R. C.; Graessley, W. W. Macromolecules 1999, 32, 3502-3509.

35. Baumgaertel, M.; De Rosa, M. E.; Machado, J.; Masse, M.; Winter, H. H. Rheol. Acta. 1992, 31, 75-82.

36. Roovers, J. Polymer Journal (Tokyo, Japan) 1986, 18, 153-162.

37. Raju, V. R.; Menezes, E. V.; Marin, G.; Graessley, W. W.; Fetters, L. J. Macromolecules 1981, 14, 1668-1676. 


\title{
Chapter 4
}

\section{Semidilute Solution Rheology for Turbulent Drag Reduction Phenomenology}

\begin{abstract}
As an application of universal scaling of semidilute polymer solutions, we investigated the shear-dependent rheological properties of poly (ethylene oxide) solutions for reducing turbulent drag in boundary layer on the wall-surface. Even though drag reduction research begins with dilute solutions, we need to also consider semidilute regime because relative drag reduction to Newtonian flow increases with the square root of concentration, ${ }^{1}$ and formation of aggregates of polymers diminishes the overlap concentration at which semidilute regime begins; see Figure 4.1. Non-Newtonian flow with a long chain polymer dissolved in it shows a significant drop in friction as the polymer chain in the flow suppreses turbulence with increasing Reynolds number. But, there exists an upper limit to the maximum drag reduction we can get, which is called Maximum Drag Reduction line (MDR). Beyond the onset Reynolds number for drag reduction, the friction dramatically reduces until it reaches MDR; the higher the absolute slope, the more quickly we obtain MDR.
\end{abstract}




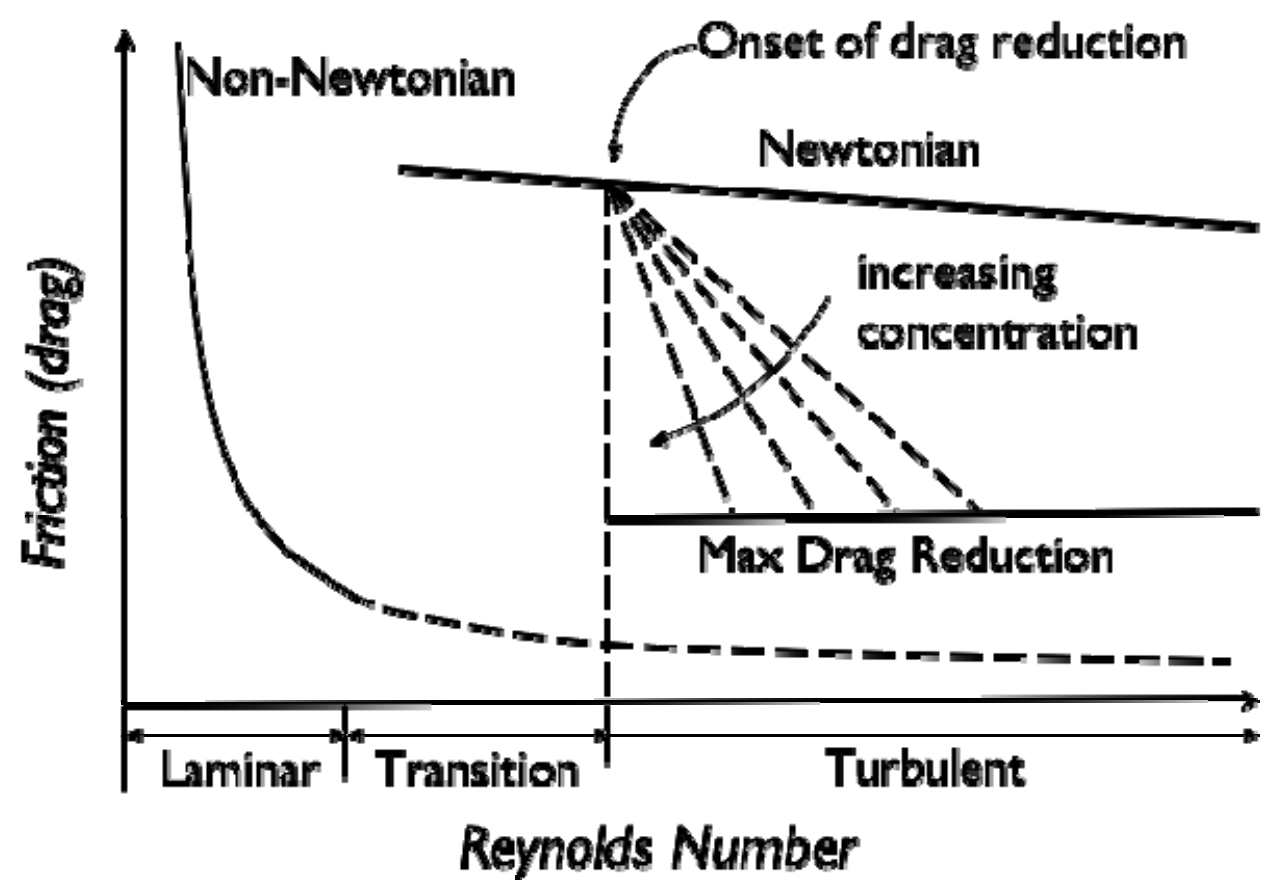

Figure 4.1. Schematic of the concentration effect on the relative drag reduction to Newtonian flow. Beyond the onset of drag reduction in Reynolds number, polymer solutions with higher concentration will reach the maximum drag reduction more quickly.

\subsection{Introduction}

Since the relaxation time scale of polymers can be comparable to that of eddy in flow, polymer chains can have an impact on the macroscopic flow properties of polymer solutions. Drag reduction is one of these examples, which have been extensively studied in many disciplines since Toms (1949)²; high extensional viscosity near the wall due to stretching of polymer by turbulence disrupts eddy momentum transport in the wall normal direction resulting in drag reduction. As the molecular conformation of a polymer chain in a flow determines the probability of interaction with neighbor polymer chains, polymers with linear structure and higher molecular weight (around $10^{6} \mathrm{~g} / \mathrm{mol}$ ) have greater chances of entanglement leading to higher proportion of reduction in drag at a given concentration. ${ }^{3}$ Due to this, commercial poly (ethylene oxide), Polyox WSR 301, 
308, N60K with molecular weight of 4 million, 8million, and 2 million $\mathrm{g} / \mathrm{mol}$ have been commonly used for drag reduction research. To describe the dynamics of these poly (ethylene oxide) (PEO) solutions, Finitely Extensible Nonlinear Elastic model with Peterlin's closure (FENE-P), a reasonable compromise between reality and simplicity, was used. Even though this model is only capable of obtaining the longest relaxation time of the polymer in solution as compared to FENE model with multiple relaxation time spectra, a single relaxation time is known to be sufficient to qualitatively capture the features of turbulent drag reduction in the near-wall region. ${ }^{4,5}$ Shown below is the FENEP constitutive equation.

$$
\stackrel{\nabla}{\mathbf{S}}+\frac{1}{\lambda}\left(\frac{\mathbf{S}}{1-(\operatorname{tr} \mathbf{S}) / R_{0}^{2}}-\left(R_{0}^{2} / b\right) \mathbf{I}\right)=0
$$

where $\mathbf{S}=\langle\mathbf{R R}\rangle$ with $\mathbf{R}$ the vector between two beads (the angular brackets indicate a configuration average), $\lambda$ is the longest relaxation time of the polymer solution, $R_{0}$ is the maximal possible extension of the polymer chain, $b$ is a measure of the extensibility of the polymer chain modeled as a dumbbell, formulated $b=H R_{0}^{2} / k_{B} T$ with $H$ the spring constant, and $k_{B}$ the Boltzmann constant. In steady shear flow, the shear-dependent viscosity $\eta$ can be predicted using this constitutive equation as given below.

$$
\eta=\eta_{s}+\eta_{p}=\eta_{s}+v k_{B} T \cdot \lambda \cdot \frac{b}{\left(4 \lambda^{2} \gamma^{2} b^{2}\right)^{1 / 3}} \cdot\left(\Delta_{1}^{1 / 3}+\Delta_{2}^{1 / 3}\right)
$$

where, $\eta_{p}$ is the polymer contribution to the zero-shear viscosity,

$\eta_{s}$ the solvent viscosity, $v$ is the number of polymer chains in a unit volume, 


$$
\Delta_{1}=1+\sqrt{1+\frac{2(b+3)^{3}}{27 \lambda^{2} \gamma^{\prime} b^{2}}} \text { and } \Delta_{2}=1-\sqrt{1+\frac{2(b+3)^{3}}{27 \lambda^{2} \gamma^{2} b^{2}}} \text {. }
$$

Here, $\eta_{p}, \eta_{s}, b$, and $\lambda$ are the fitting parameters of FENE-P equation. With this equation, in principle, we obtain not only steady shear viscosity, but also the first normal stress difference and the storage and loss moduli, which are the essential rheological properties of polymer solutions. Thus, determining the above-listed fitting parameters is a foremost task for characterizing the flow of drag-reducing polymer solutions.

\subsection{Sample Preparations}

We prepared WSR301 and WSR308 PEO solutions of highest required concentration with HPLC grade water. To prevent shear degradation, we dissolved PEO powder in water by rotating the solution-containing bottles on a roller at 3 6 rpm. We allowed 48 hours for dissolution using this method. After this, we diluted some of the samples by a factor of 2 and 4 to get solutions with lower concentrations.

\subsection{Steady Shear Viscosity Measurements}

As explored by Toms $(1949)^{2}$, dilute polymer solutions with only a few parts per million can effectively reduce the drag up to $80 \%$. It is also well known that longer polymer chains lead to more reduction in drag due to early overlap and entanglement effect at a given concentration. To confidently determine the zero-shear viscosity of a dilute polymer solution, we need at lease one decade of low shear rate plateau region. Thus, Contraves Low Shear 30 rheometer (Contraves) with bob radius of $R_{i}=5.5 \mathrm{~mm}$, cup inner 
radius of $R_{0}=6 \mathrm{~mm}$, bob length of $L=20 \mathrm{~mm}$ was used for the shear rates up to $100(1 / \mathrm{s})$. As explained in Chapter 2, this rheometer is sensitive enough to measure a zero-shear viscosity of around $2 \mathrm{cP}$ at a shear rate of $0.017(1 / \mathrm{s}){ }^{6}$ Two more rheometers, AR1000 and ARES (by Shaqfeh group at Stanford University) were also used for higher shear rates; see Figure 4.2.

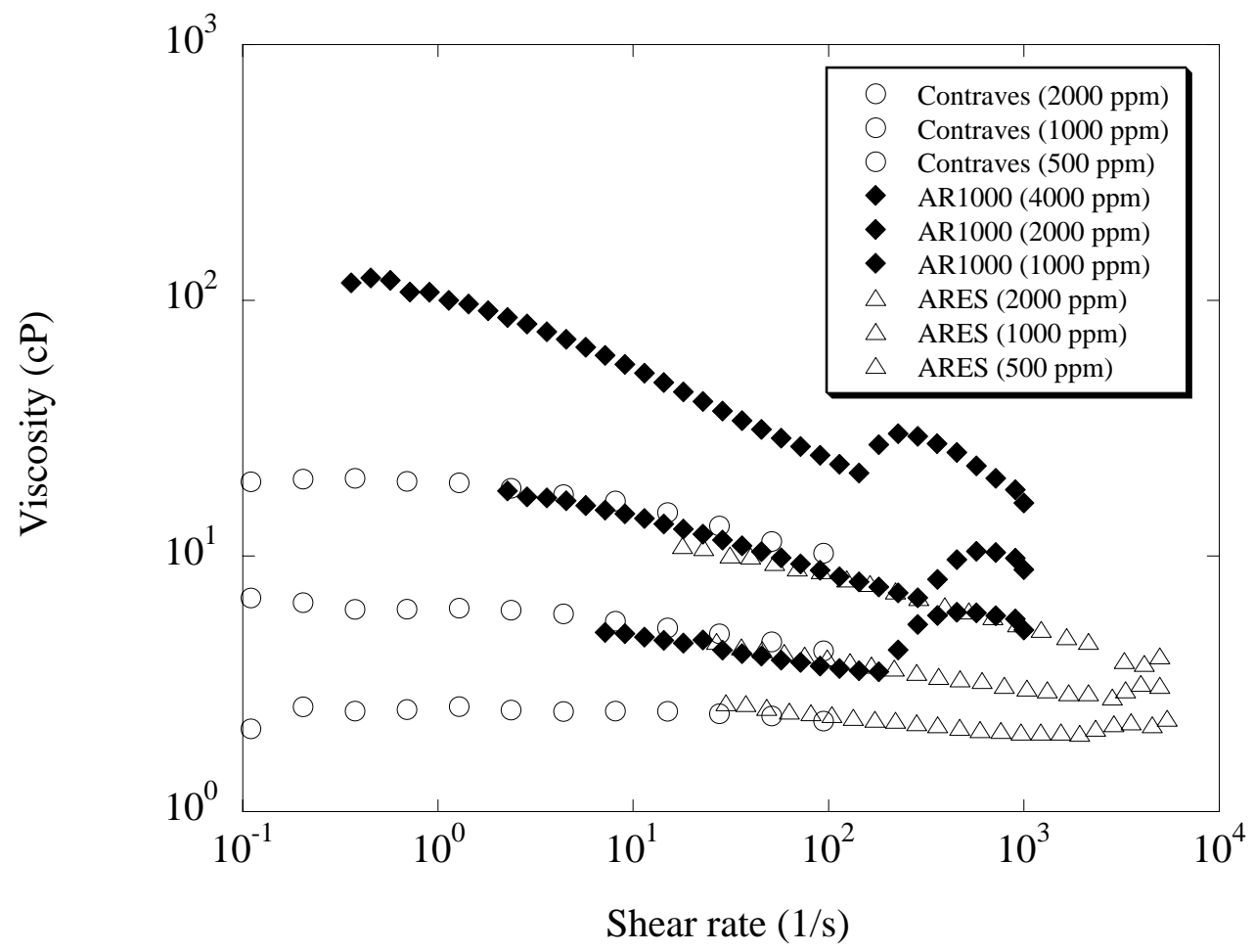

Figure 4.2. Shear-dependent viscosities of WSR301 PEO solutions at four concentrations with three different rheometers. The concentrations shown next to the rheometer used in the legend correspond to the shear-dependent viscosities from top to bottom.

As illustrated in Figure 4.2, these results are reasonably consistent with each other from three different rheometers. The pronounced upturn at high shear rates by AR1000 results from elastic instability, of which onset is dependent on the geometry of a fixture. 


\subsection{Constitutive Equations}

\subsubsection{FENE-P Fitting with Simulated Annealing}

We used Simulated Annealing (SA) method to obtain the fitting parameters of FENE-P to experimental data. SA is a heuristic algorithm for approximating the global optimum of a given function inspired by thermodynamic annealing in metallurgy. This procedure is robust because uphill moves are allowed by Metropolis acceptance probability, $p=\exp \left[-\left(E_{1}-E_{2}\right) / k_{B} T\right]$, to avoid getting trapped in local minima. ${ }^{7,8}$

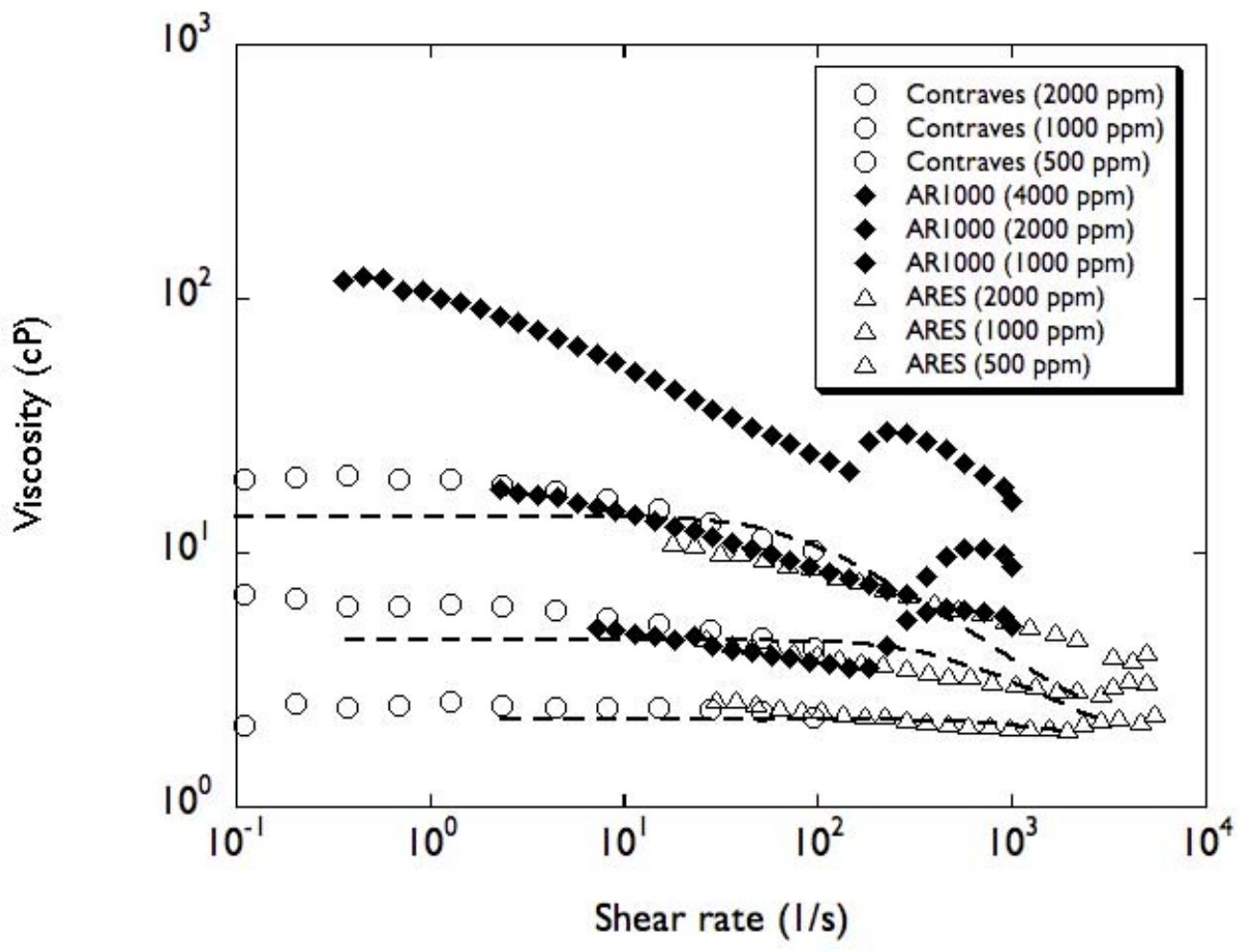

Figure 4.3. FENE-P fitting (dashed lines) with $b$ and $\eta_{s}$ fixed to the combined experimental data of WSR 301. Note that we were not able to acquire the FENE-P fit to $4000 \mathrm{ppm}$ data due to the lack of sufficient plateau region. 
It is reasonable to fix two fitting parameters $b$ and $\eta_{s}$ because they physically mean the extensibility and the solvent viscosity, which are not supposed to vary over shear rates. However, we were not able to obtain a reasonably good fit to the combined experimental data set with these two parameters fixed as demonstrated in Figure 4.3; the zero-shear viscosities of WSR 301 samples are consistently higher than the FENE-P predictions. Although the viscosities of solutions at different concentrations are supposed to converge to the viscosity of its solvent at high shear rates, we observe that the "solvent viscosity" grows with concentration of a solution.

\subsubsection{Modified FENE-P}

To overcome the aforementioned anomalous behavior of shear dependent viscosities of PEO solutions, D. T. Walker fit data for all concentrations of a given polymer simultaneously with cleverly chosen power-law functional forms for the relaxation time $\lambda$, the polymer contribution to zero-shear viscosity $\eta_{p}$, and the "solvent viscosity" $\eta_{s}$.

$$
\begin{gathered}
\eta_{p}=\eta_{w}\left([\eta]_{0} c\right)^{n} \\
\eta_{s}=\eta_{w}+\eta_{w}\left([\eta]_{0} c\right)^{m} \\
\lambda=a\left([\eta]_{0} c\right)^{k} \\
b=5.23 \times 10^{-3} M_{w}
\end{gathered}
$$

Here, $\eta_{w}$ is the viscosity of water, $[\eta]_{0}$ is the intrinsic viscosity of a PEO solution, and $n, m, a$, and $k$ are the fitting parameters of modified FENE-P model. The "solvent viscosity" obtained from this fit is not the water viscosity, but another variable that grows 
with polymer concentration. It actually represents an apparent high-shear rate plateau viscosity obtained at shear rates of $1000 \mathrm{~s}^{-1}$ or so. To see how these viscosities for PEO compare with those of other polymer solutions, we employed the "universal scaling curve" for the zero-shear viscosity as a function of concentration. ${ }^{6}$ To construct this curve, we collected multiple data sets of zero-shear viscosity versus concentration for semidilute polymer solutions in good solvents both from literature and from our own experiments. We found that all experimental data including a non-aggregated PEO solution ${ }^{9}$ above a critical concentration $c / c_{e}>0.5$ fall on a single empirical curve given by $\eta_{p} / \eta_{\text {Rouse }}=(43 \pm 2) \times\left(c / c_{e}\right)^{3.12 \pm 0.05}$ with $v$ the excluded volume exponent, $\eta_{p}=\eta_{0}-\eta_{s}$ the polymer contribution to the zero shear viscosity of the solution with $\eta_{0}$ the zero-shear viscosity and $\eta_{s}$ the actual solvent viscosity, and with $\eta_{\text {Rouse }}=\eta_{s}\left(c[\eta]_{0}\right)^{1 /(3 v-1)}$ the hypothetical Rouse polymer viscosity, and $c_{e}=n_{e}^{3 v-1} /\left(M_{w} A_{2}\right)$ the entanglement concentration of the polymer solution; see Figure 4.4. Here, $n_{e}$ is the number of "blobs" per entanglement and can be obtained from $n_{e} \cong N_{e} \equiv M_{e} / M_{0}$ with $N_{e}$ the number of entanglements per molecule in melts, $M_{e}$ the entanglement molecular weight, and $M_{0}$ the monomer molecular weight. ${ }^{6,10-13}$

We now wish to place the data for PEO solutions on this plot. To get the entanglement crossover concentration, $c_{e}$, we need the entanglement molecular weight, $M_{e}$ for which two values for PEO have been given in the literature, namely $1624 \mathrm{~g} / \mathrm{mol}^{14}$ and 4400 g/mol. ${ }^{15}$ 


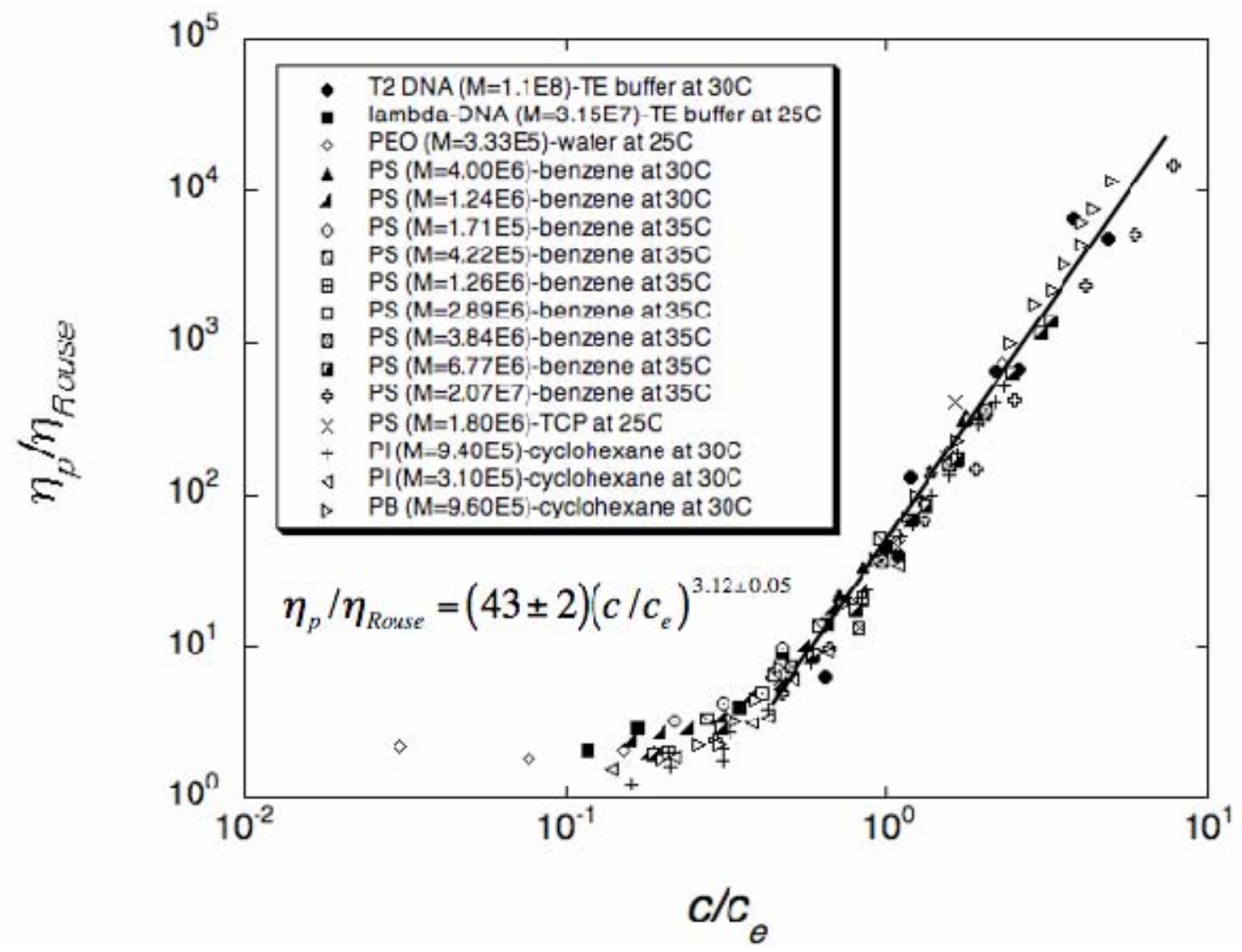

Figure 4.4. Experimental data points of semidilute polymer solutions in good solvents lying on a single universal plot, $\eta_{p} / \eta_{\text {Rouse }}=(43 \pm 2) \times\left(c / c_{e}\right)^{3.12 \pm 0.05}$

In Figure 4.5, it is shown that the zero-shear viscosities of low-molecular-weight, presumably non-aggregated PEO solutions studied by van Zanten et al., ${ }^{9}$ collapse better onto the universal curve with the choice with $M_{e}=4400$ than with $M_{e}=1624$. However, the zero-shear viscosities of WSR 301, 308 and N60K from Walker's fits, using $M_{e}=4400$ and the intrinsic viscosity of Bailey et al. $(1959)^{16}$ do not fall on the universal curve; see the black filled symbols on Figure 4.6. Use of the other value of $M_{e}=1624$ brings the PEO zero-shear viscosities closer to the universal curve but they still lie well above it. 


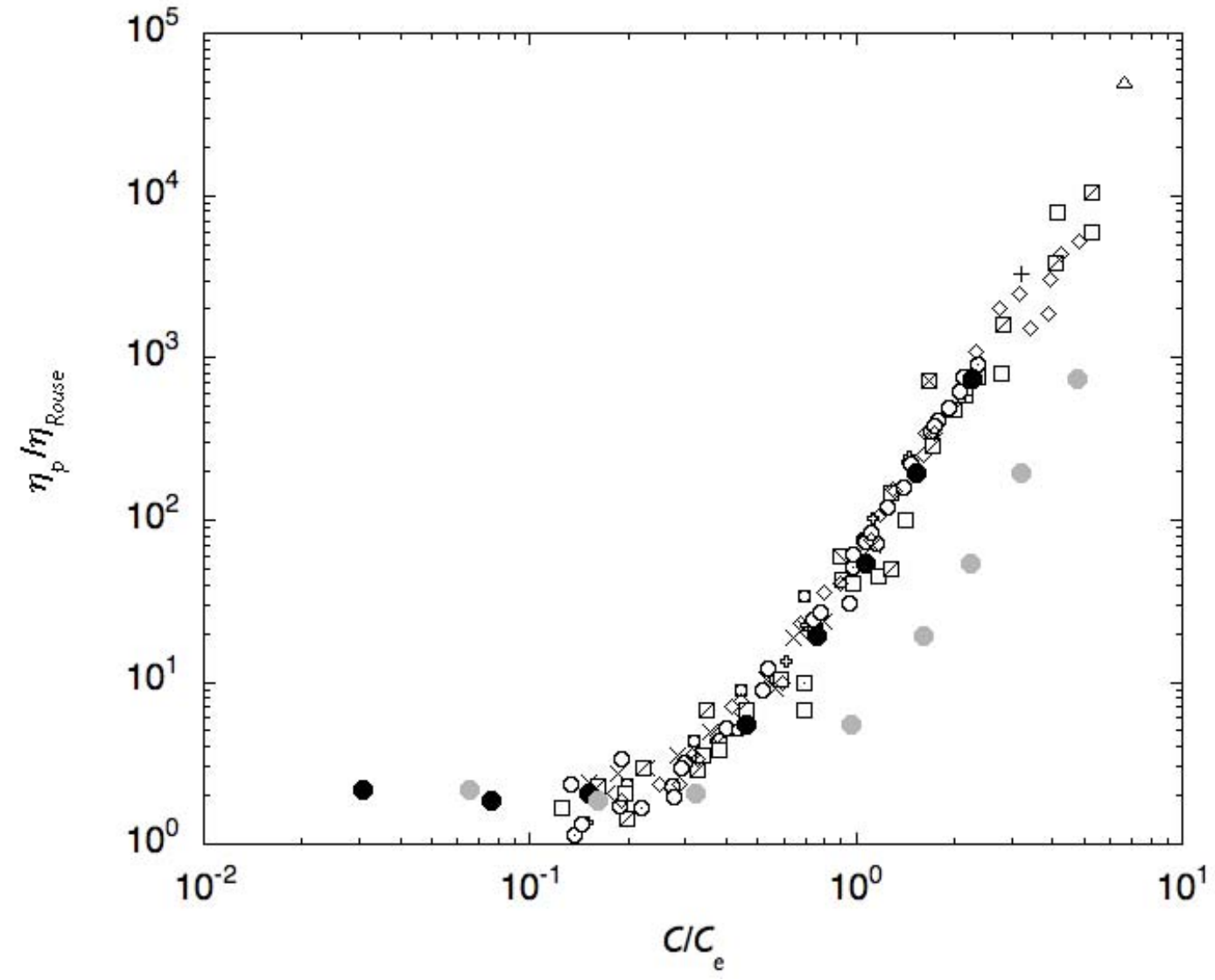

Figure 4.5. Comparison Non-aggregated PEO $\left(M_{w}=3.33 \times 10^{5}\right)$ solutions with two different entanglement molecular weights, $M_{e}=4400 \mathrm{~g} / \mathrm{mol}$ (black filled circles) and $M_{e}=1624 \mathrm{~g} / \mathrm{mol}$ (gray filled circles).

Studies by the Solomon, Hanratty, and others indicate that PEO molecules of high molecular weight (above a million) are aggregated in aqueous solution and this is consistent with the high value of the zero-shear viscosity relative to the expected value for non-aggregated solutions given by universal curve. However, we note that the "solvent viscosities" for these solutions, which are the filled red symbols on Figure 4.6 and are calculated by Eq. 4 (illustrated in Figure 4.7), fall on the universal curve. 


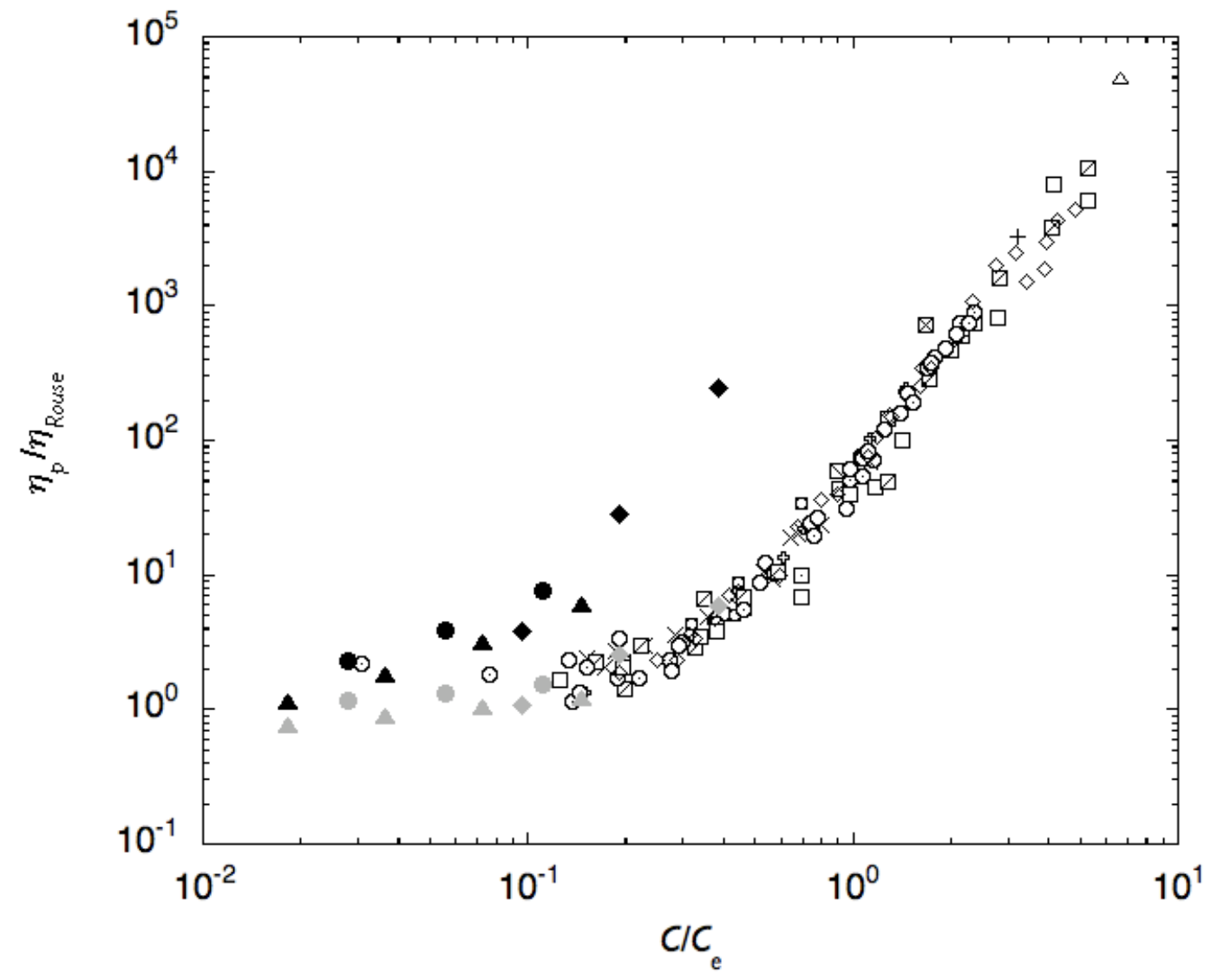

Figure 4.6. Comparison of zero-shear viscosities (black filled symbols) and "solvent viscosities" (gray filled symbols) of WSR 301 (circles), 308 (diamonds), and N60K (triangles) on the universal curve.

This indicates that when these solutions are sheared, the aggregates are either broken down or their contribution to the viscosity is suppressed, so that the apparent "solvent viscosity” for these solutions is the actual zero-shear viscosity that these solutions would have if they were not aggregated. 


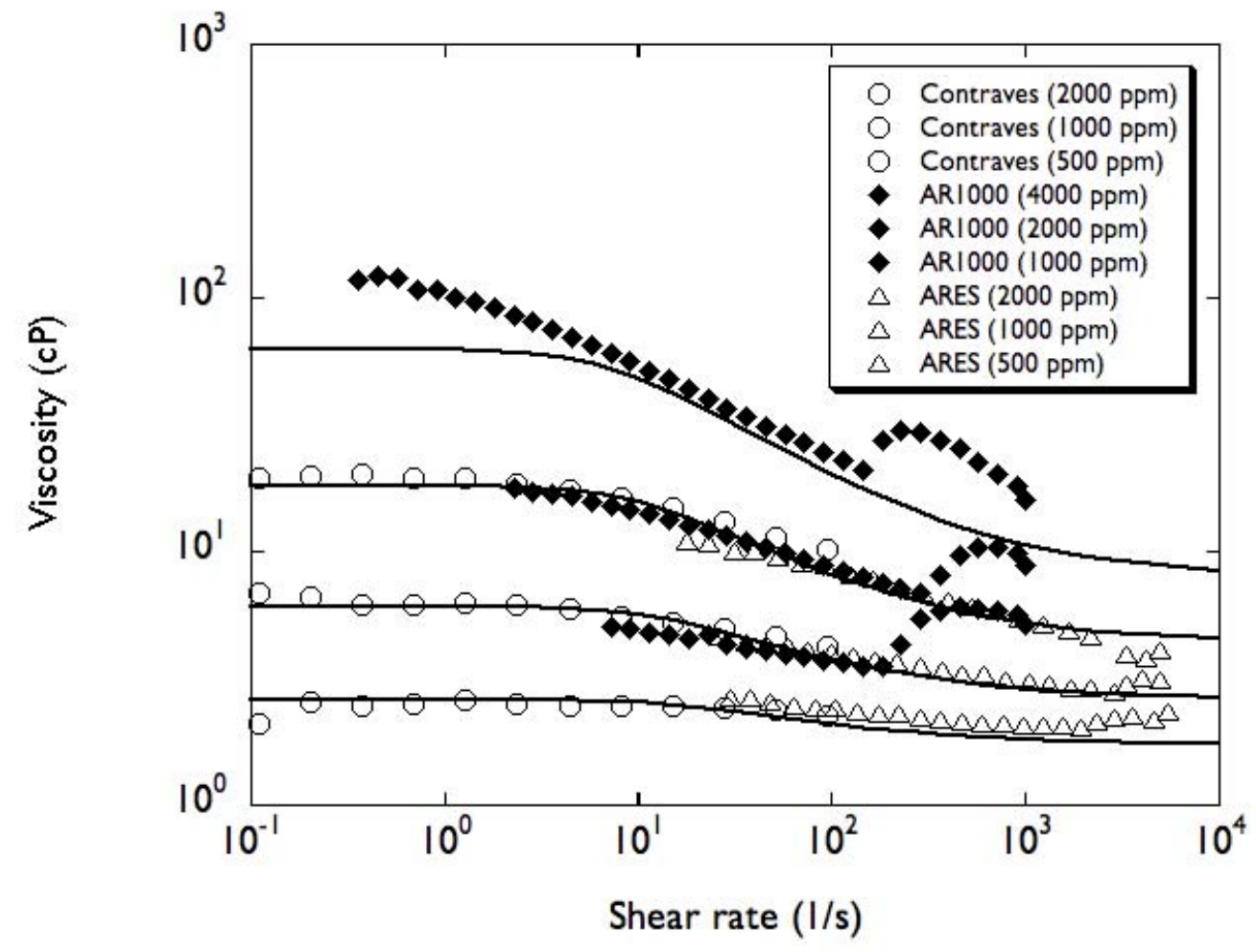

Figure 4.7. Modified FENE-P fitting by utilizing Eqs. 3-6.

\subsection{Future Direction}

High values of zero-shear viscosities and growing solvent viscosities indicate aggregation of polymers in semidilute regime. When these solutions are sheared, the aggregates are either broken down or their contribution to the viscosity is suppressed. Thus the apparent " solvent viscosity” for these solutions is the actual zero-shear viscosity that these solutions would have, if they were not aggregated.

This work motivates the need to explore the structure of PEO solutions to elucidate the mechanism of aggregate formation and breakage. 


\section{References}

1. Roy, A.; Larson, R. G. Applied Rheology, 2005, 15, 370-389.

2. Toms, B. A. Proc. First Intern. Congr. On Rheology, 1948, 2, 135-141.

3. Truong, V. -T. Drag Reduction Technologies, DSTO Aeronautical and Maritime Research Laboratory: Australia, 2001.

4. Purnode, B.; Crochet, M. J. J. Non-Newtonian Fluid Mech. 1998, 77, 1-20.

5. Jin, S.; Collins, L. R. New Journal of Physics, 2007, 9,1-22.

6. Heo, Y.; Larson, R. G. J. Rheology, 2005, 49, 1117-1128.

7. Corana, A.; Marchesi, M.; Martini, C.; Ridella, S. ACM Trans. Math. Softw, 1987, 13, 262-280.

8. Goffe, W. L.; Ferrier, D. G.; Rogers, J. J. Econometrics 1994, 60, 65-99.

9. van Zanten, J. H.; Amin, S.; Abdala, A. A. Macromolecules 2004, 37, 3874-3880.

10. Menezes, E. V.; Graessley, W. W. J. Polym. Sci., Polym. Phys. Ed. 1982, 20, 18171833.

11. Raspaud, E.; Lairez, D.; Adam, M. Macromolecules 1995, 28, 927-933

12. Osaki, K.; Inoue, T.; Uematsu, T.; Yamashita, Y. J. Polym. Sci., Part B: Polym. Phys. 2001, 39, 1704-1712.

13. Heo, Y.; Larson, R. G. J. Rheology 2007, 51, 1099-1100.

14. Fetters, L. J.; Lohse, D. J.; Richter, D. Witten, T. A.; Zirke, A. Macromolecules 1994, 27, 4639-4647.

15. Dontula, P.; Macosko, C. W.; Scriven, L. E. AIChE J. 1998, 44, 1247-1255.

16. Bailey. F. E.; Kucera, J. L.; Imhof, L. G. J. Poly. Sci. 1958, 32, 517-518. 


\section{Chapter 5}

\section{Summary and Future Work}

\section{1. Summary}

In this dissertation, we have tried to establish the relationship of a polymer chain conformation in solution and the rheological properties of this solution in the light of de Gennes’ blob scaling. The dimension and dynamics of a polymer chain is significantly affected by its thermodynamics, such as excluded volume interaction between polymer segments. Even though the excluded volume exponent ( $v$ in $R_{g} \sim M^{v}$ ) for a polymer solution does not seem to change considerably over solvent quality ( $v=0.5$ for polymers in $\Theta$ solvent and $v=0.588$ for asymptotically good solvent), the magnitude of difference will be enormous with high molecular weight polymers as illustrated in Figure 5.1. An imaginary polymer chain with $10^{10}$ monomers of size $1 \mathrm{~cm}$ in four different interactions are shown; in poor solvent, the chain fits a classroom, in $\Theta$ solvent, it fits a campus, in good solvent, it fits the size of a city, and the contour length of a chain will be a quarter of the distance to the moon. 


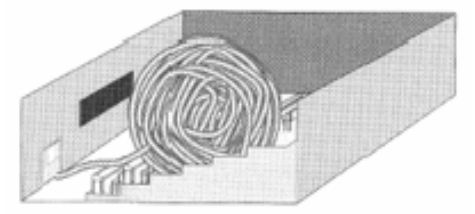

attractive interaction

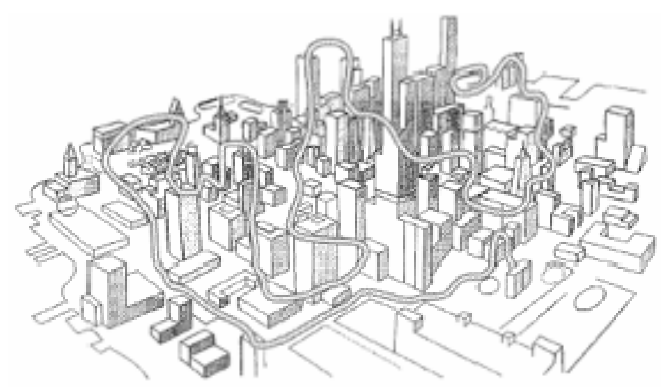

short-range repulsion

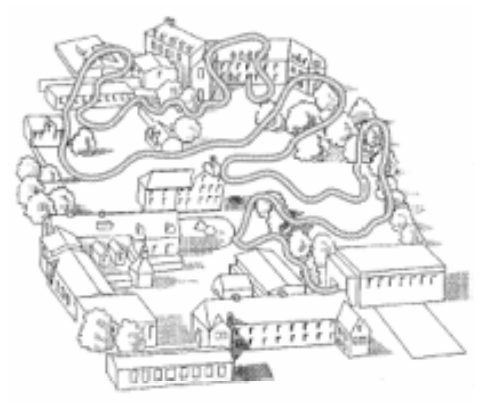

zero effective interaction
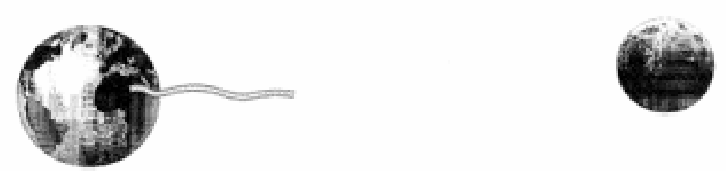

long-range repulsion

Figure 5.1. Dimension of a polymer chain in solution according to the quality of solvent used; see text. ${ }^{1}$

Therefore, we suggested that the true solvent quality, which is determined from intrinsic viscosity measurement $[\eta]_{0}=K M^{a}$ with $K$ and $a=3 v-1$ the Mark-Houwink parameters, should be taken into account when dealing with chain dimension in semidilute regime. For linear polymers, as long as the number of correlation blobs is the same, the dynamic similarity is expected, and has been tested by linear and nonlinear rheological measurements.

Zero-shear viscosity, which is a measure of the resistance of a fluid to shear strain in terminal region, was first employed to test the dynamic similarity of flexible synthetic polymers and semiflexible DNA solutions. As shown in Chapter 2, we verified that a universal scaling law exists for normalized zero-shear viscosities versus normalized concentrations. 
Second, the complex moduli, and shear dependent viscosity and first normal stress coefficient of monodisperse polymer solutions, in general, turned out to follow another universal scaling law. However, we had to determine the correct value or range of the swelling volume fraction $\phi_{s}$ at which a polymer chain dimension shifts in scaling relation because the thermal fluctuation becomes dominant over swelling effect of polymers in a good solvent as volume fraction of polymer increases. We demonstrated two different cases of polymers: polystyrene/tricresyl phosphate and polybutadiene/phenyl octane solutions. Since there is no sharp transition in scaling relation, determining precise value of $\phi_{s}$ from rheological measurements is very difficult. Therefore, we examined a potential range of volume fractions where universal scaling starts to break down, and choose one volume fraction that makes the plots look good without violating the order of $\mathrm{G}^{\prime}$ or $\mathrm{G}^{\prime \prime}$ location with regard to the entanglement density. In addition, two bidisperse polymer solutions with almost the same ratio of long to short chain were investigated as a first step for exploring polydiperse systems.

Third, semidilute solution rheology for turbulence drag reduction was studied as an application of universal scaling of zero-shear viscosities with concentrations. Since FENE-P fitting model did not work for WSR 301 and WSR 308 PEO solutions with anomalous behaviors such as much higher zero-shear viscosities than predicted by FENE-P, and the growing "solvent viscosity" with concentration, modified fitting parameters were suggested. These anomalies indicate aggregation of PEO in semidilute regime, in parallel with two peaks observed in the distribution function of dynamic light scattering data. When these solutions are sheared, the aggregates are either broken down or their contribution to the viscosity is suppressed. Thus, the apparent "solvent viscosity" 
for these solutions is the actual zero-shear viscosity that these solutions would have, if they were not aggregated.

\subsection{Future Work}

To construct the universal scaling incorporating two different scaling regimes by utilizing Milner (2005) ${ }^{2} \mathrm{~s}$ formulas, we need to develop more precise methods for evaluating the swelling volume fraction $\phi_{s}$ not only from scaling relation, but also with a prefactor subsumed in it. Besides this, as indicated in Chapter 3, when the monomeric friction factors of a polymer segment and the solvent are substantially separated, polymer solutions have growing friction with volume fraction even in semidilute regime. These findings are in contrast with de Gennes, ${ }^{3}$ assumption that the friction difference within semidilute regime is negligible. Thus, if we become able to locate the correct value of $\phi_{s}$, and estimate an appropriate value of monomeric firction for a correlation blob in a polymer solution, we would expect more perfect version of universal scaling relation. Furthermore, it will relocate the data points of the universal scaling plot of zero-shear viscosities with concentrations because zero-shear viscosity is affected by the friction factor as demonstrated in Colby et al. (1991), ${ }^{4}$ and some of the excluded volume exponents that we used for polymer/solvent pairs need to be changed depending on which scaling regime the solution belongs to. More comprehensive dynamic light scattering study is also required to unravel the aggregation phenomena of semidilute PEO solutions.

Potential applications of this universal scaling relationship include the mapping of the molecular weight and concentration of initial polymer solutions with the morphologies of electrospun fibers. There exist numerous electrospinning process parameters such as 
conductivity, flow rate, the distance between tip and collector, and so on, which are combined to govern the final morphology of a fiber. ${ }^{5}$ Therefore, we possibly obtain a different morphology with a different combination of process parameters listed above, even though we employ the same initial polymer concentration. To establish the structure-property relationships of a polymer/solvent pair for electrospinning, instead of considering this large number of potential combinations of process parameters, we need to determine a range of each parameter with which electrified jet is induced. Then, we decide a set of process parameters that allow us to explore several different morphologies depending on the concentration and molecular weight of a polymer in solution. We expect that the universal dependence of zero-shear viscosity of a polymer solution on its concentration can characterize the initial number of correlation blobs, and the normalized viscosity $\eta_{p} / \eta_{\text {Rouse }}$ tells us the morphology of the final product.

Scaling of diblock copolymer solutions would be another good application of "blob" scaling model. In melt, a diblock copolymer self-assembles to form a certain morphology according to the length ratio of two components. In solution, we expect that counting the number of correlation blobs for each component can predict the morphology that the block copolymer solution might take. In order to prove this idea, we can do rheological measurements on these solutions by increasing the length of one component with the other fixed within the boundary where a single morphology dominates. And, we can check whether the same scaling relationship as in melt state exhibits. 


\section{References}

1. Rubinstein, M.; Colby, R. H. Polymer Physics, Oxford University Press Inc., New York, 2003.

2. Milner, S. T. Macromolecules 2005, 38, 4929-4939.

3. de Gennes, P. -G. Scaling Concepts in Polymer Physics; Cornell University Press: Ithaca, New York, 1979.

4. Colby, R. H.; Fetters, L. J.; Funk, W. G.; Graessley, W. W. Macromolecules 1991, 24,3873-3882.

5. Pham, Q. P.; Sharma, U.; Mikos, A. G. Tissue Engineering 2006, 12, 1197-1211. 


\section{Appendix}

\section{Combined Universal Scaling of Polystyrene Solutions in Tricresyl Phosphate}

As illustrated in Chapter 3, there exists gradual breakdown of de Gennes, ${ }^{1}$ universal scaling as the volume fraction goes above about $15 \%$ of polystyrene (PS) solutions in tricresyl phosphate (TCP). This is presumably because of the transition in scaling regime from semidilute, which follows de Gennes’ (DG) blob scaling, to concentrated, which follows Colby-Rubinstein (CR) scaling relation ${ }^{2}$. Although we are not allowed to pinpoint the exact value of the swelling volume fraction $\phi_{s}$, which separates DG and CR regimes, we would like to investigate how the storage and loss moduli of PS/TCP solutions with different values of $\phi_{s}$ align in the order of entanglement density $N / N_{e}$ with $N$ the number of monomers, and $N_{e}$ the number of monomers needed for a polymer chain in solution to have an entanglement.

\section{Entanglement Density}

The entanglement density of a polymer chain in a good solvent is

$$
N / N_{e}(\phi)=\left\{\begin{array}{l}
{\left[N / N_{e}(1)\right] \phi_{s}^{\alpha_{c}}\left(\phi / \phi_{s}\right)^{1 /(3 v-1)}, \phi<\phi_{s},} \\
{\left[N / N_{e}(1)\right] \phi^{\alpha_{c}}, \phi_{s}<\phi}
\end{array}\right.
$$


where, $\alpha_{c}$ is the universal dilution exponent, of which value is $4 / 3^{2}, v$ is the excluded volume exponent ranging from 0.5 to 0.588 depending the solvent quality, and $N_{e}(\phi)$ is

$$
N_{e}(\phi)=\left\{\begin{array}{l}
N_{e}(1) \phi_{s}^{-\alpha_{c}}\left(\phi / \phi_{s}\right)^{1 /(1-3 v)}, \phi<\phi_{s} \\
N_{e}(1) \phi^{-\alpha_{c}}, \phi_{s}<\phi
\end{array}\right.
$$

\section{Normalizing Parameters}

To align the storage and loss moduli of PS/TCP solutions at $25^{\circ} \mathrm{C}$ those of PS melts at $180^{\circ} \mathrm{C}$ on the same plot, we need to normalize their time and modulus.

Equilibration time, the normalizing parameter for time scale is represented as

$$
\tau_{e, \text { scaling }}=\left\{\begin{array}{l}
\frac{\eta_{s}}{k_{B} T} b^{3} N_{e}^{2}(1) \phi_{s}^{1-2 \alpha_{c}}\left(\phi / \phi_{s}\right)^{3 v /(3 v-1)}, \phi<\phi_{s} \\
\frac{\eta_{s}}{k_{B} T} b^{3} N_{e}^{2}(1) \phi_{s}^{1-2 \alpha_{c}}, \phi_{s}<\phi
\end{array},\right.
$$

where $\eta_{s}$ the solvent viscosity, $k_{B}$ the Boltzmann constant, and $b$ the effective monomer step length.

For the plateau modulus, the normalizing parameter for modulus is expressed as

$$
G_{N}^{0}(\phi)=\left\{\begin{array}{l}
G_{N}^{0}(1) \phi_{s}^{1+\alpha_{c}}\left(\phi / \phi_{s}\right)^{3 v /(3 v-1)}, \phi<\phi_{s} \\
G_{N}^{0}(1) \phi^{1+\alpha_{c}}, \phi_{s}<\phi
\end{array}\right.
$$

Table A.1 lists the values of all parameters that we employ to calculate the entanglement density and normalizing parameters, and Table A.2 itemizes the volume fraction, molecular weight, equilibration time, and plateau modulus in the descending order of entanglement density $N / N_{e}(\phi)$ with three different swelling volume fractions: $\phi_{s}=0.09$, 0.12 , and 0.15 . For polybutadiene $(\mathrm{PBd})$ solutions in phenyl octane $(\mathrm{PhO}), K_{1}$ is used as a constant that links $\tau_{e, \text { scaling }}$ and $\tau_{e, D E}$ since the friction factors of this polymer, and that of 
the solvent are very close, leading to no significant change of friction with volume fraction. However, in case of PS/TCP solutions, $K_{1}$ becomes a function of volume fraction once it exceeds a certain volume fraction beyond which the solvent viscosity no longer dominates the friction of a correlation blob. This change in friction with volume fraction of polymer results from the large difference between the glass transition temperatures of PS and TCP, which affects the friction $\left(T_{g, P S}=373 \mathrm{~K}\right.$ and $\left.T_{g, T C P}=210 \mathrm{~K}\right)$.

Thus, we estimate the value of $K_{1}(\phi)$ by matching the high frequency Rouse region of the loss modulus of a solution with that of melts. We then obtain a correlation between $K_{1}(\phi)$ $\phi$, which we fit by an exponential and apply this formula to get the value of $\tau_{e, D E}(\phi)=K_{1}(\phi) \tau_{e, \text { scaling }}(\phi)$ for each polymer solution.

Table A.1. Parameters for calculating Eqs. (1)-(4).

\begin{tabular}{|c|c|c|c|c|c|c|c|}
\hline $\begin{array}{c}k_{B} \\
\mathrm{~m}^{2} \cdot \mathrm{kg} \cdot \mathrm{s}^{-2} \cdot \mathrm{K}^{-1}\end{array}$ & $\begin{array}{l}T \\
K\end{array}$ & $\alpha_{c}$ & $\begin{array}{r}\eta_{s} \\
c P\end{array}$ & $v^{\mathrm{a}}$ & $\begin{array}{l}b^{b} \\
n m\end{array}$ & $\begin{array}{c}G_{N}^{0}(1)^{\mathrm{C}} \\
P a\end{array}$ & $N_{e}(1)^{\mathrm{d}}$ \\
\hline $1.38 \times 10^{-23}$ & 289 & $4 / 3$ & 58 & 0.53 & 0.67 & $2.23 \times 10^{5}$ & 125 \\
\hline
\end{tabular}

${ }^{a}$ ref. 3

bef. 4

c ref. 5

${ }^{\mathrm{d}} N_{e}(1)=M_{e}(1) / M_{0}$ with $M_{e}(1)=12,960 \mathrm{~g} / \mathrm{mol}^{4}$ and $M_{0}=104 \mathrm{~g} / \mathrm{mol}$

Table A.2. Entanglement density and normalizing parameters depending on different swelling volume fraction.

(1) $\phi_{s}=0.09: K_{1}(\phi)=0.7881 \cdot \exp (8.1111 \phi)$

\begin{tabular}{|c|c|c|c|c|}
\hline$N / N_{e}(\phi)$ & $\phi$ & $\begin{array}{c}M_{w} \\
10^{6} \mathrm{~g} / \mathrm{mol}\end{array}$ & $\begin{array}{c}\tau_{e, \text { scaling }}(\phi) \\
\text { s }\end{array}$ & $\begin{array}{c}G_{N}^{0}(\phi) \\
P a\end{array}$ \\
\hline 20.9 & 0.091 & 6.62 & $5.37 \times 10^{-3}$ & $8.31 \times 10^{2}$ \\
\hline 17.0 & 0.160 & 2.53 & $5.59 \times 10^{-3}$ & $3.10 \times 10^{3}$ \\
\hline 15.1 & 0.075 & 6.62 & $8.34 \times 10^{-3}$ & $4.95 \times 10^{2}$ \\
\hline 14.6 & 0.239 & 1.28 & $6.08 \times 10^{-3}$ & $7.90 \times 10^{3}$ \\
\hline 13.3 & 0.133 & 2.53 & $3.80 \times 10^{-3}$ & $2.01 \times 10^{3}$ \\
\hline
\end{tabular}




\begin{tabular}{|c|l|l|l|l|}
\hline 11.5 & 0.199 & 1.28 & $4.86 \times 10^{-3}$ & $4.12 \times 10^{3}$ \\
\hline 9.2 & 0.056 & 6.62 & $1.96 \times 10^{-2}$ & $2.25 \times 10^{2}$ \\
\hline 8.8 & 0.098 & 2.53 & $6.32 \times 10^{-3}$ & $9.87 \times 10^{2}$ \\
\hline 7.7 & 0.089 & 2.53 & $7.51 \times 10^{-3}$ & $7.85 \times 10^{2}$ \\
\hline 7.6 & 0.146 & 1.28 & $4.88 \times 10^{-3}$ & $2.50 \times 10^{3}$ \\
\hline 7.4 & 0.049 & 6.62 & $3.38 \times 10^{-2}$ & $1.57 \times 10^{2}$ \\
\hline 6.6 & 0.131 & 1.28 & $3.90 \times 10^{-3}$ & $1.94 \times 10^{3}$ \\
\hline 4.5 & 0.065 & 2.53 & $1.40 \times 10^{-2}$ & $3.37 \times 10^{2}$ \\
\hline 4.4 & 0.036 & 6.62 & $4.31 \times 10^{-2}$ & $6.85 \times 10^{1}$ \\
\hline 4.2 & 0.094 & 1.28 & $6.78 \times 10^{-3}$ & $8.96 \times 10^{2}$ \\
\hline 2.2 & 0.024 & 6.62 & $9.63 \times 10^{-2}$ & $2.30 \times 10^{1}$ \\
\hline 2.1 & 0.061 & 1.28 & $1.04 \times 10^{-2}$ & $2.84 \times 10^{2}$ \\
\hline 2.0 & 0.040 & 2.53 & $3.24 \times 10^{-2}$ & $9.10 \times 10^{1}$ \\
\hline
\end{tabular}

(2) $\phi_{s}=0.12: K_{1}(\phi)=0.7881 \cdot \exp (8.1111 \phi)$

\begin{tabular}{|c|c|c|c|c|}
\hline$N / N_{e}(\phi)$ & $\phi$ & $\begin{array}{c}M_{w} \\
10^{6} \mathrm{~g} / \mathrm{mol}\end{array}$ & $\begin{array}{c}\tau_{e, \text { scaling }}(\phi) \\
\text { s }\end{array}$ & $\begin{array}{c}G_{N}^{0}(\phi) \\
P a\end{array}$ \\
\hline 18.9 & 0.091 & 6.62 & $8.56 \times 10^{-3}$ & $6.39 \times 10^{2}$ \\
\hline 17.0 & 0.160 & 2.53 & $8.38 \times 10^{-3}$ & $2.63 \times 10^{3}$ \\
\hline 14.6 & 0.239 & 1.28 & $1.02 \times 10^{-2}$ & $6.72 \times 10^{3}$ \\
\hline 13.6 & 0.075 & 6.62 & $1.80 \times 10^{-2}$ & $3.79 \times 10^{2}$ \\
\hline 13.3 & 0.133 & 2.53 & $7.13 \times 10^{-3}$ & $1.71 \times 10^{3}$ \\
\hline 11.5 & 0.199 & 1.28 & $6.56 \times 10^{-3}$ & $4.38 \times 10^{3}$ \\
\hline 8.3 & 0.056 & 6.62 & $3.70 \times 10^{-2}$ & $1.73 \times 10^{2}$ \\
\hline 8.2 & 0.098 & 2.53 & $1.34 \times 10^{-2}$ & $7.80 \times 10^{2}$ \\
\hline 7.6 & 0.146 & 1.28 & $1.03 \times 10^{-2}$ & $2.13 \times 10^{3}$ \\
\hline 7.0 & 0.089 & 2.53 & $1.51 \times 10^{-2}$ & $6.02 \times 10^{2}$ \\
\hline 6.6 & 0.049 & 6.62 & $6.81 \times 10^{-2}$ & $1.20 \times 10^{2}$ \\
\hline 6.6 & 0.131 & 1.28 & $7.31 \times 10^{-3}$ & $1.65 \times 10^{3}$ \\
\hline 4.1 & 0.065 & 2.53 & $3.00 \times 10^{-2}$ & $2.58 \times 10^{2}$ \\
\hline 3.9 & 0.036 & 6.62 & $9.55 \times 10^{-2}$ & $5.25 \times 10^{1}$ \\
\hline 3.9 & 0.094 & 1.28 & $1.50 \times 10^{-2}$ & $6.97 \times 10^{2}$ \\
\hline 2.0 & 0.024 & 6.62 & $2.07 \times 10^{-1}$ & $1.76 \times 10^{1}$ \\
\hline 1.9 & 0.061 & 1.28 & $2.52 \times 10^{-2}$ & $2.17 \times 10^{2}$ \\
\hline 1.8 & 0.040 & 2.53 & $7.19 \times 10^{-2}$ & $6.97 \times 10^{1}$ \\
\hline
\end{tabular}

(3) $\phi_{s}=0.15: K_{1}(\phi)=0.6118 \cdot \exp (9.5611 \phi)$

\begin{tabular}{|l|l|l|l|l|}
\hline$N / N_{e}(\phi)$ & $\phi$ & $M_{w}$ & $\tau_{e, \text { scaling }}(\phi)$ & $G_{N}^{0}(\phi)$ \\
\hline
\end{tabular}




\begin{tabular}{|c|c|c|c|c|}
\hline & & $10^{6} \mathrm{~g} / \mathrm{mol}$ & $s$ & $P a$ \\
\hline 17.5 & 0.091 & 6.62 & $5.98 \times 10^{-3}$ & $6.93 \times 10^{2}$ \\
\hline 17.0 & 0.160 & 2.53 & $1.40 \times 10^{-3}$ & $3.10 \times 10^{3}$ \\
\hline 14.6 & 0.239 & 1.28 & $7.16 \times 10^{-4}$ & $7.90 \times 10^{3}$ \\
\hline 12.7 & 0.133 & 2.53 & $2.15 \times 10^{-3}$ & $1.93 \times 10^{3}$ \\
\hline 12.6 & 0.075 & 6.62 & $1.01 \times 10^{-2}$ & $4.12 \times 10^{2}$ \\
\hline 11.5 & 0.199 & 1.28 & $9.71 \times 10^{-4}$ & $5.16 \times 10^{3}$ \\
\hline 7.7 & 0.056 & 6.62 & $2.21 \times 10^{-2}$ & $1.87 \times 10^{2}$ \\
\hline 7.6 & 0.098 & 2.53 & $4.90 \times 10^{-3}$ & $8.47 \times 10^{2}$ \\
\hline 7.5 & 0.146 & 1.28 & $1.67 \times 10^{-3}$ & $2.48 \times 10^{3}$ \\
\hline 6.4 & 0.089 & 2.53 & $6.35 \times 10^{-3}$ & $6.53 \times 10^{2}$ \\
\hline 6.3 & 0.131 & 1.28 & $2.24 \times 10^{-3}$ & $1.85 \times 10^{3}$ \\
\hline 6.1 & 0.049 & 6.62 & $3.17 \times 10^{-2}$ & $1.31 \times 10^{2}$ \\
\hline 3.8 & 0.065 & 2.53 & $1.48 \times 10^{-2}$ & $2.80 \times 10^{2}$ \\
\hline 3.6 & 0.036 & 6.62 & $7.28 \times 10^{-2}$ & $5.70 \times 10^{1}$ \\
\hline 3.6 & 0.094 & 1.28 & $5.48 \times 10^{-3}$ & $7.57 \times 10^{2}$ \\
\hline 1.8 & 0.024 & 6.62 & $2.17 \times 10^{-1}$ & $1.91 \times 10^{1}$ \\
\hline 1.7 & 0.061 & 1.28 & $1.76 \times 10^{-2}$ & $2.36 \times 10^{2}$ \\
\hline 1.7 & 0.040 & 2.53 & $5.48 \times 10^{-2}$ & $7.57 \times 10^{1}$ \\
\hline
\end{tabular}

Figures A.1-3 illustrate how the scaling plots change with different choice of swelling volume fraction $\phi_{s}$. The sequence of entanglement densities shown in legends is in the order of descending terminal time. Therefore, one can easily check whether the entanglement density calculated from Milner's expression leads to the proper monotonic ordering of the rescaled $G^{\prime \prime}(\omega)$ curves along the frequency axis as $N / N_{e}(\phi)$ is varied. 

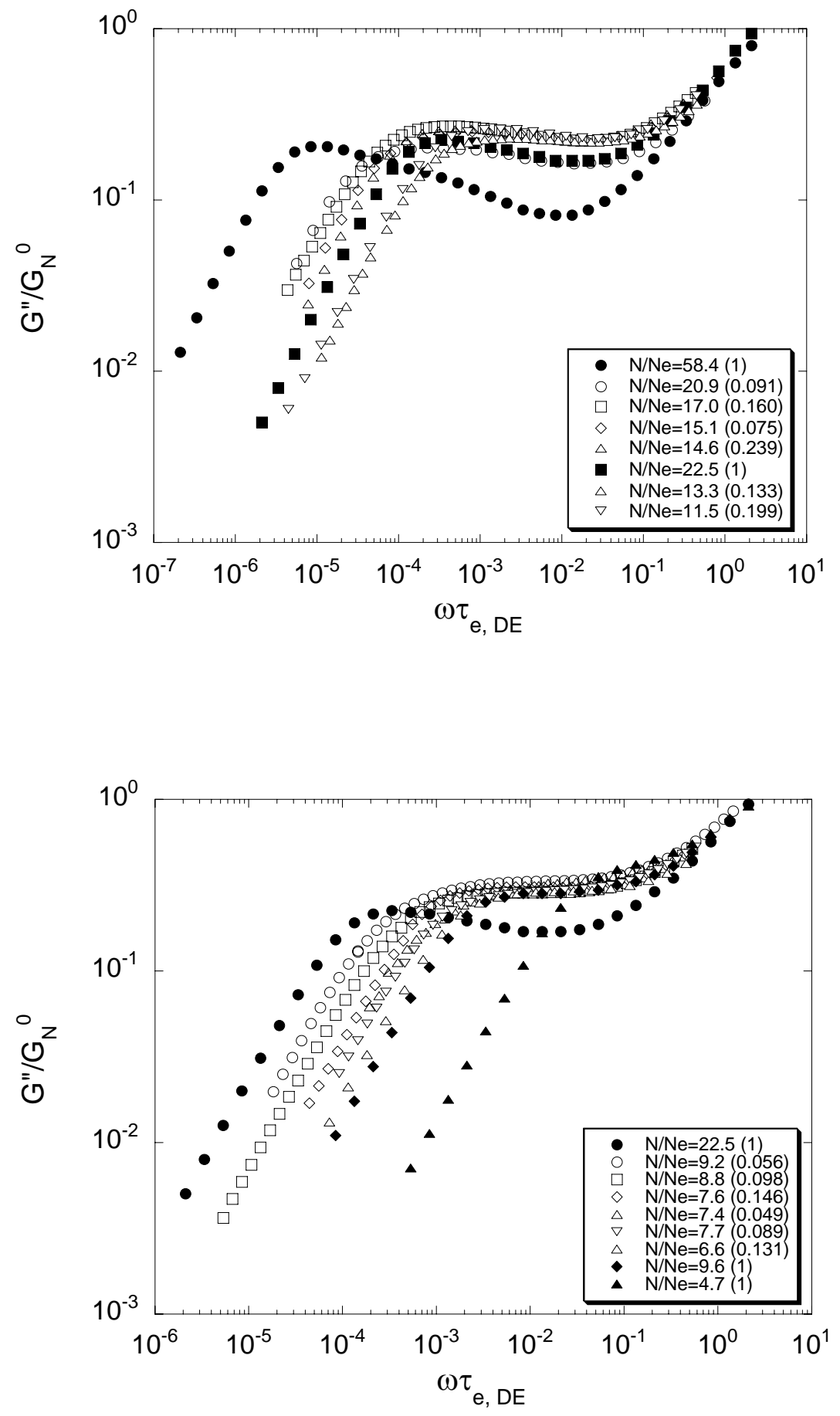

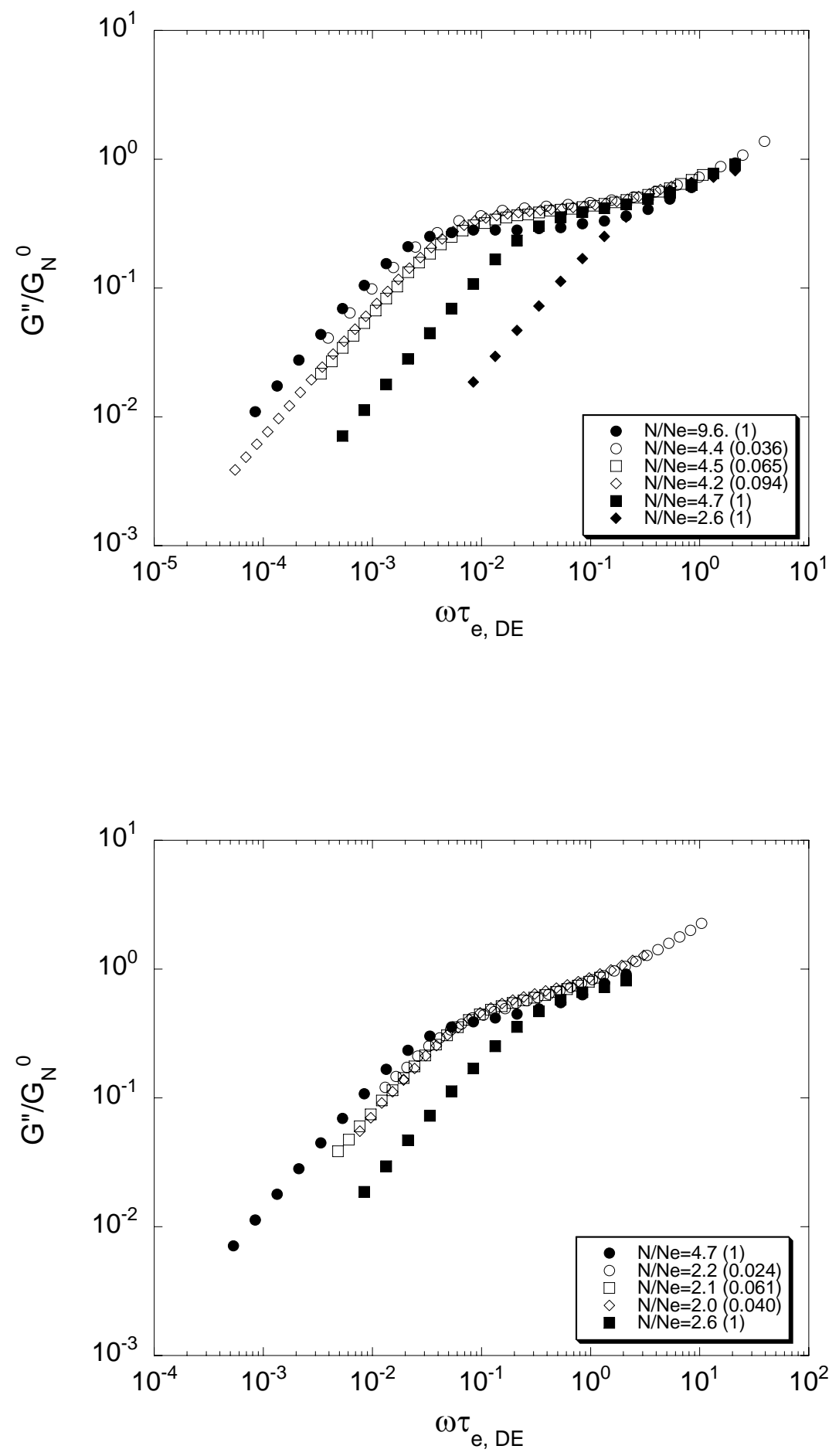

Figure A.1. Normalized loss modulus versus normalized frequency of PS melts and PS/TCP solutions with $\phi_{s}=0.09$. Filled and open symbols respectively represent melts and solutions 

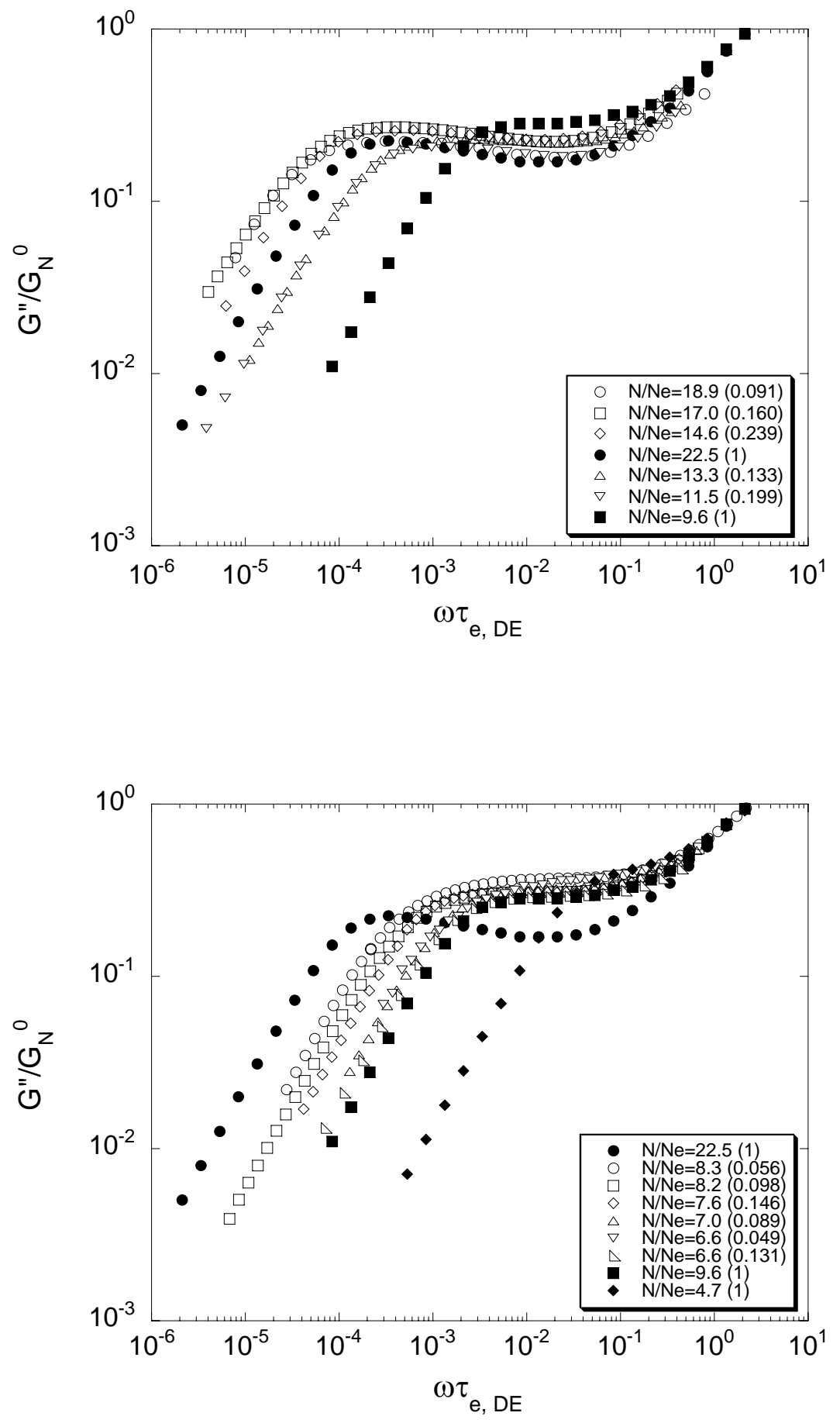

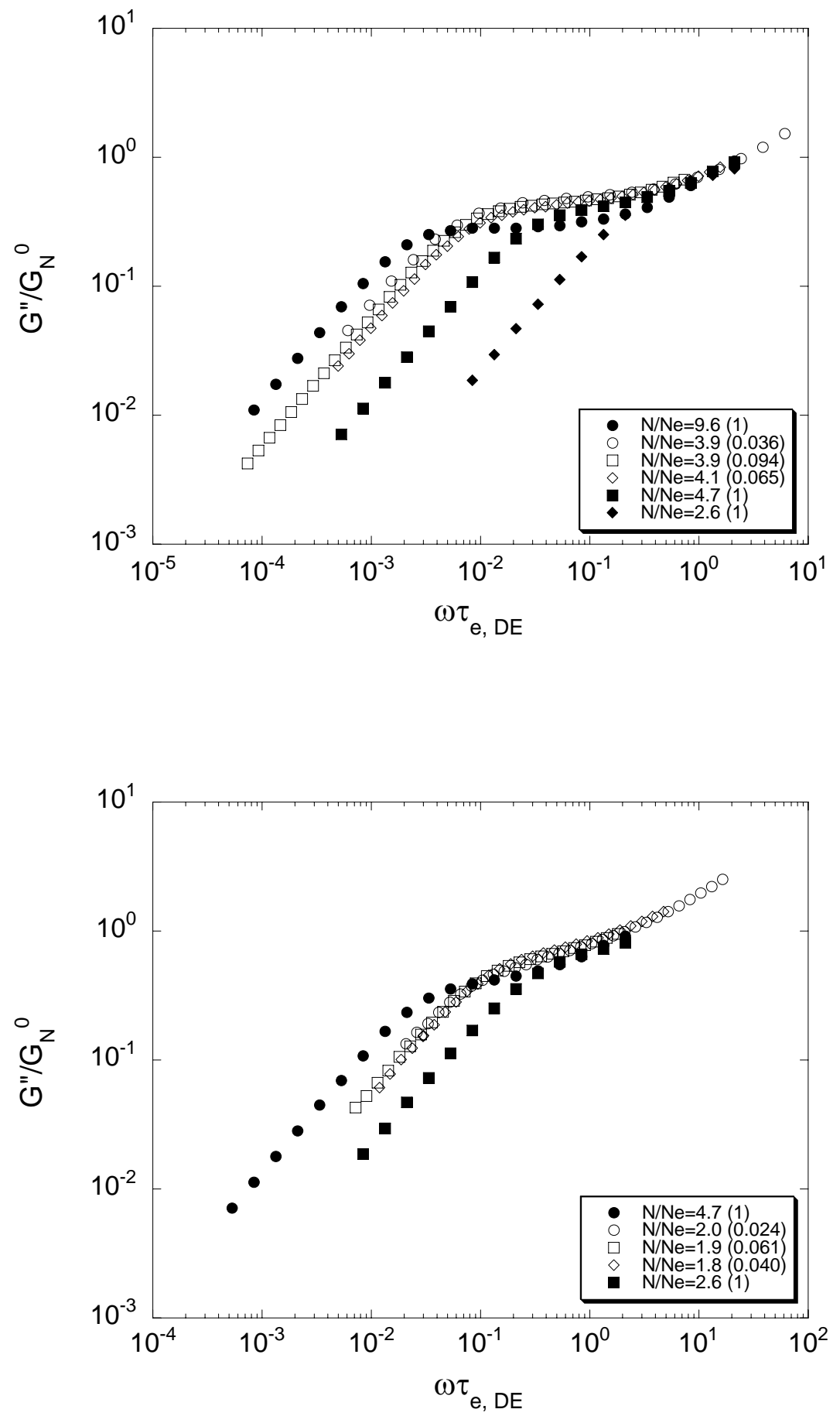

Figure A.2. Normalized loss modulus versus normalized frequency of PS melts and PS/TCP solutions with $\phi_{s}=0.12$. Filled and open symbols respectively represent melts and solutions. 

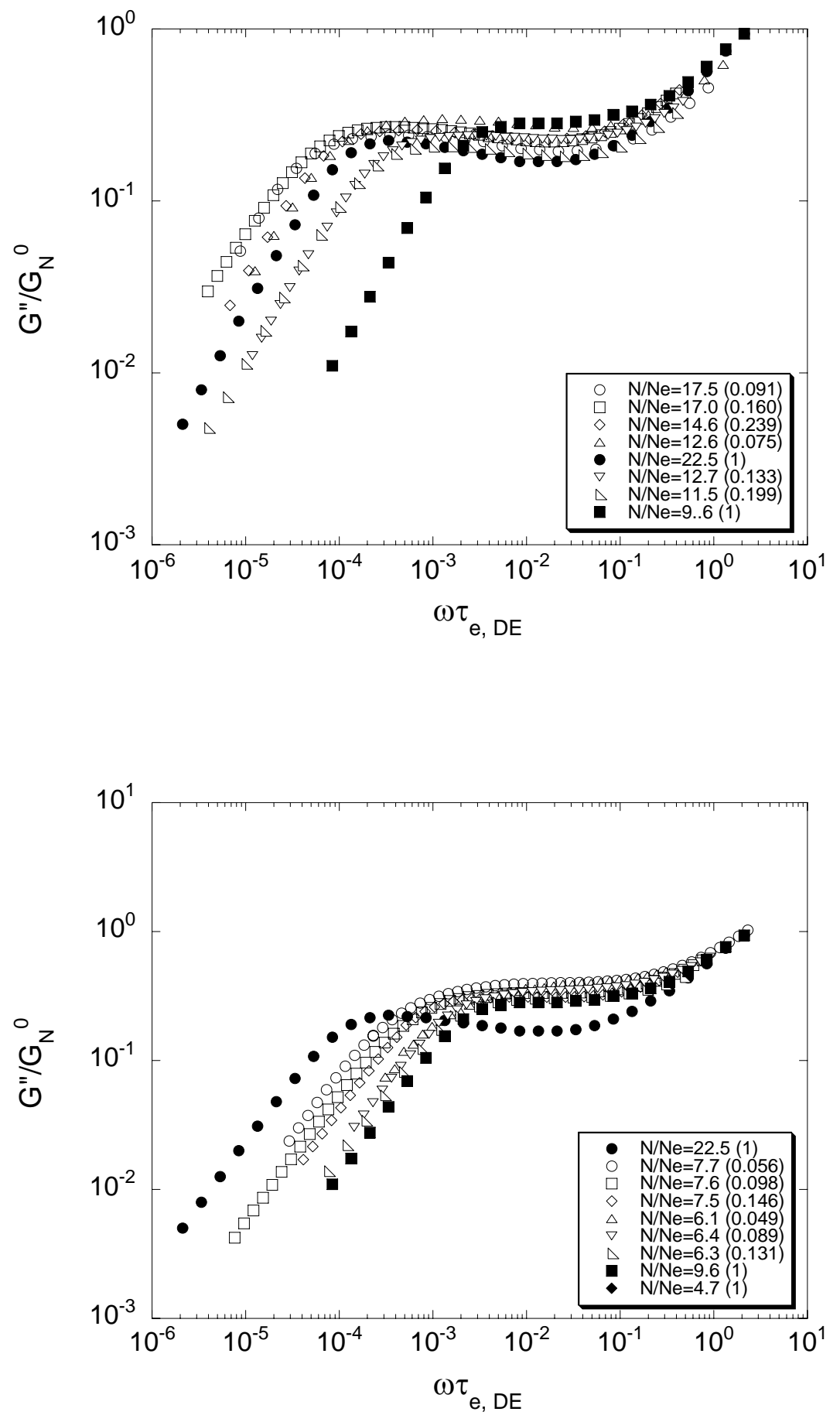

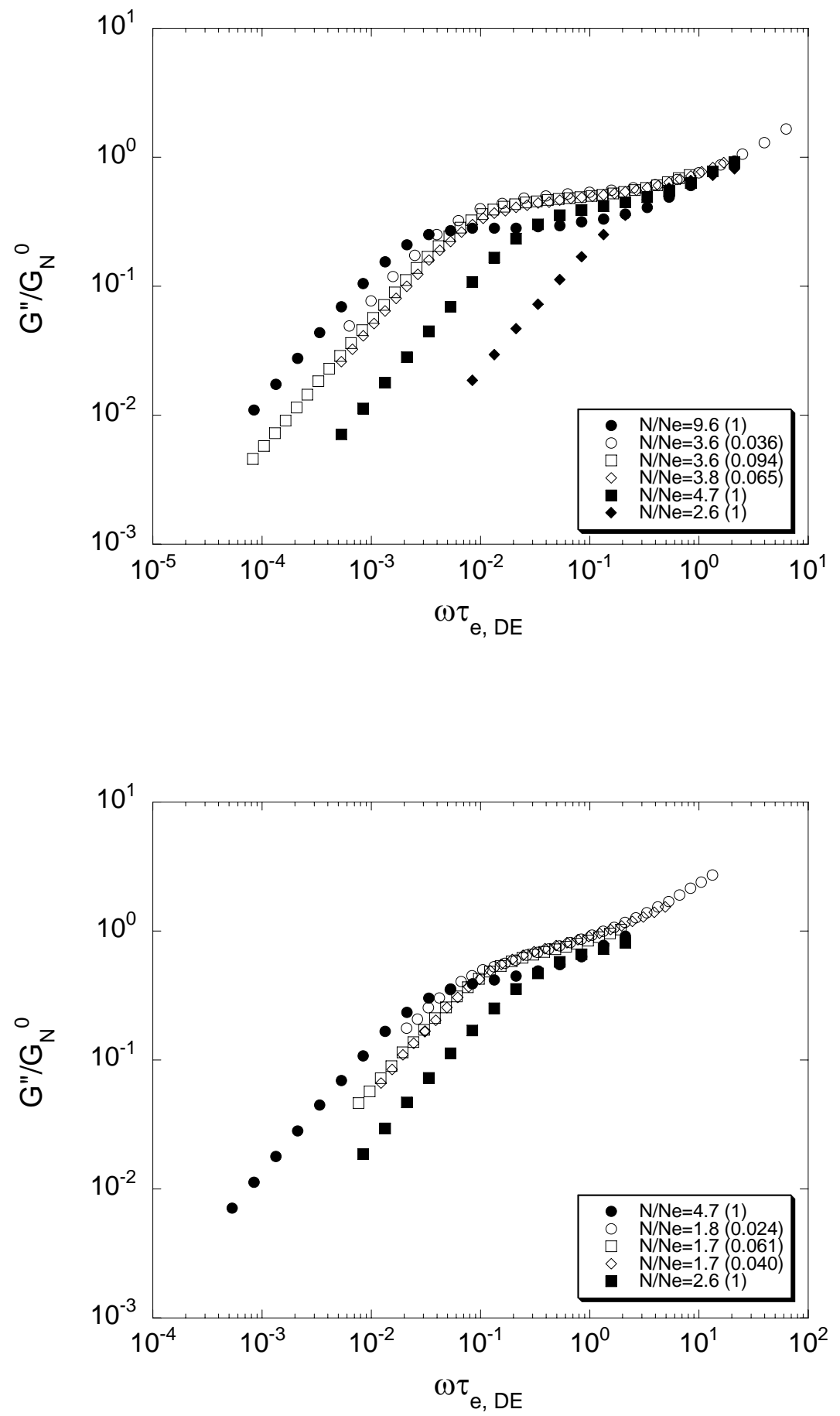

Figure A.3. Normalized loss modulus versus normalized frequency of PS melts and PS/TCP solutions with $\phi_{s}=0.15$. Filled and open symbols respectively represent melts and solutions 
In general, the entanglement densities of PS/TCP solutions seem to be underestimated, while the rescaled plateau moduli are overestimated. There appears to be no value of $\phi_{s}$ that brings rescaled $G^{\prime \prime}(\phi)$ curves for all solutions and melts data into a simple monotonic progression with increasing $N / N_{e}(\phi)$. 


\section{References}

1. de Gennes, P. -G. Scaling Concepts in Polymer Physics; Cornell University Press: Ithaca, New York, 1979.

2. Colby, R. H.; Rubinstein, M. Macromolecules 1990, 23, 2753-2757.

3. Solomon, M. J.; Muller, S. J. J. Polym. Sci., Part B: Polym. Phys. 1996, 34, 181-192.

4. Likhtman, A. E.; McLeish, T. C. B. Macromolecules 2002, 35, 6332-6343. 